\title{
Au@TiO2 Nanocomposites Synthesized by X-ray Radiolysis as Potential Radiosensitizers
}

Maria C. Molina Higgins

PhD Student

Follow this and additional works at: https://scholarscompass.vcu.edu/etd

Part of the Materials Science and Engineering Commons, Nanoscience and Nanotechnology Commons, and the Nuclear Engineering Commons

(C) The Author

\section{Downloaded from}

https://scholarscompass.vcu.edu/etd/5932

This Dissertation is brought to you for free and open access by the Graduate School at VCU Scholars Compass. It has been accepted for inclusion in Theses and Dissertations by an authorized administrator of VCU Scholars Compass. For more information, please contact libcompass@vcu.edu. 


\section{COPYRIGHT PAGE}

${ }^{\circledR} \underline{\text { Maria C. Molina Higgins } \quad 2019}$

All Rights Reserved 
Au@ $\mathrm{TiO}_{2}$ Nanocomposites Synthesized by X-ray Radiolysis as Potential Radiosensitizers

A dissertation submitted in partial fulfillment of the requirements for the degree of Doctor of Philosophy at Virginia Commonwealth University.

By

\author{
MARIA C. MOLINA HIGGINS \\ Bachelor of Science, Universidad Nacional de Colombia, Medellín, Colombia 2014
}

Director: Dr. Jessika Rojas

Assistant Professor, Department of Mechanical and Nuclear Engineering

Virginia Commonwealth University

Richmond, Virginia

April, 2019 


\section{Acknowledgments}

This work is dedicated to my family and friends in Colombia, thanks for your love and encouragement.

I would like to thank my adviser, Dr. Jessika Rojas, for her support and encouragement through my Ph.D. program. I feel so proud to be one of your first Ph.D. students, thanks for your wise advice and infinite patience. I have really enjoyed our long weekly meetings and direction that you provided every step of the way. I would also like to thank her husband and VCU professor, Dr. Carlos Castano for being a great support in my research.

I would like to thank my committee members: Dr. Dmitry Pestov, Dr. Reza Mohammadi, Dr. Laura Padilla, and Dr. Hong Zhao, thanks for your time and appreciation for my research, your constructive criticism has made this project better.

I would like to thank the faculty, staff and graduate students of the Mechanical and nuclear engineering department at VCU. You have been always great academic and emotional support over the years.

To my friends and family in Richmond, thanks for all the words of encouragement, the jokes, the laughs and the good times, you filled my journey love and joy.

Finally, I would to thank my husband Connor Howe, this would not be possible without your love and support. 


\section{Table of contents}

Chapter 1: Motivation and Research Objectives

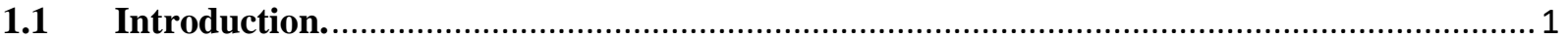

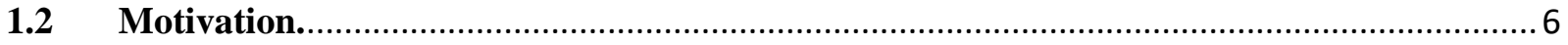

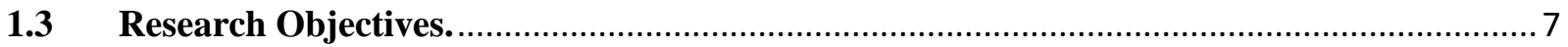

Chapter 2: Radiosensitization Background and Significance ....................................................... 13

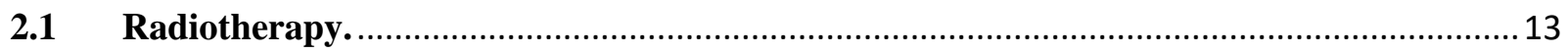

2.1.1 Physics behind radiation therapy. …................................................................. 14

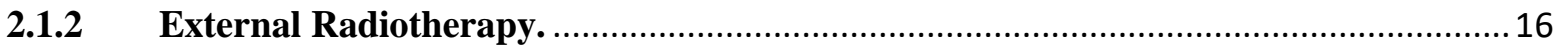

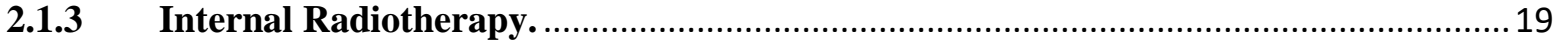

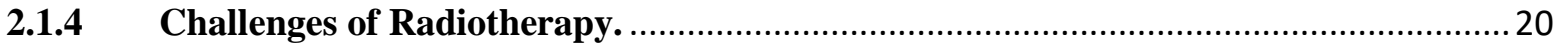

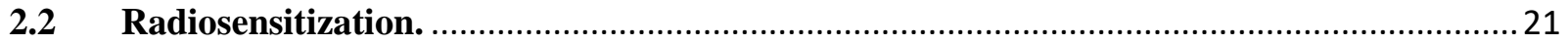

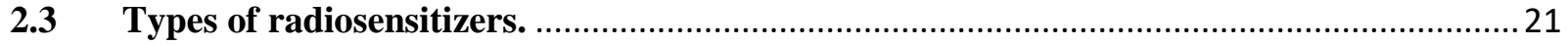

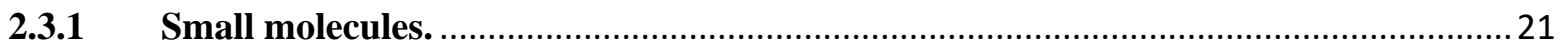

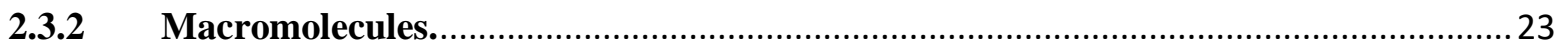

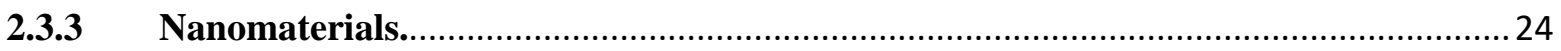

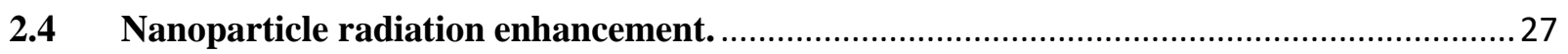

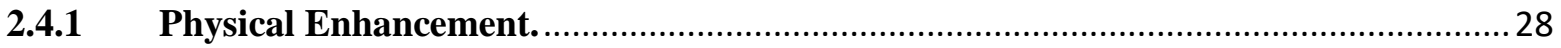

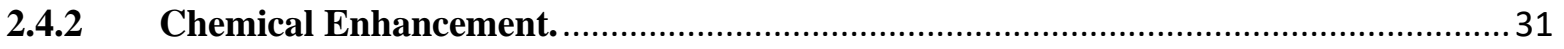

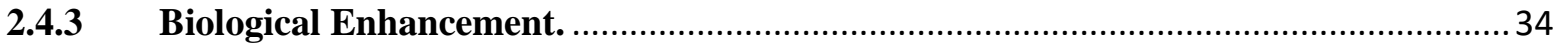

Chapter 3: Overview of fabrication methods to synthesize gold supported titania .........................36

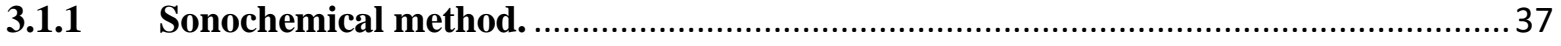


3.1.3 Deposition-precipitation Method. .38

3.1.4 Photochemical deposition method. 40

3.2 Radiolytic synthesis of nanomaterials. .......................................................................... 41

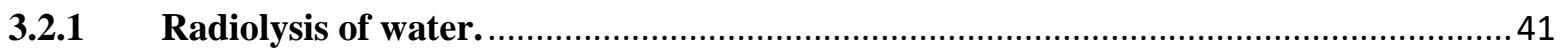

3.2.2 Nucleation and growth of Nanoparticles................................................................... 43

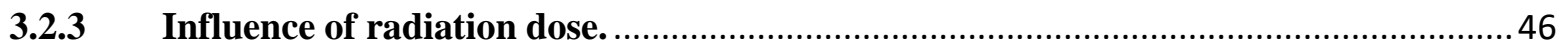

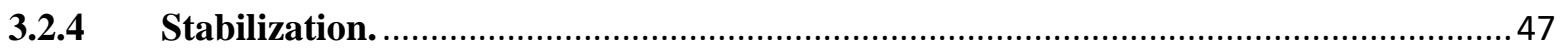

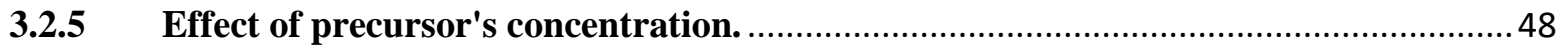

Chapter 4: X-ray radiolytic synthesis of gold supported nanoparticles ....................................... 49

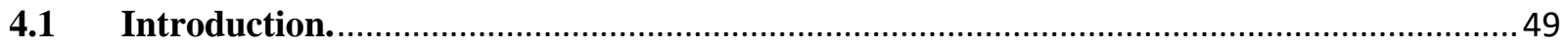

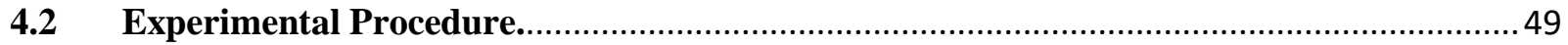

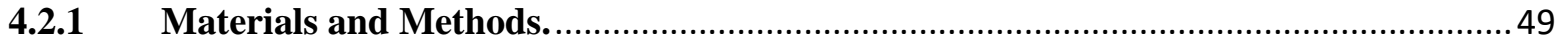

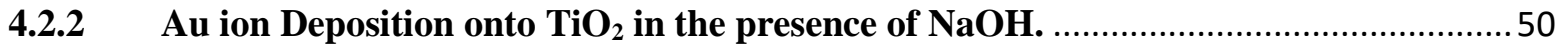

4.2.3 Au ion Deposition onto $\mathrm{TiO}_{2}$ in the presence of Urea.............................................50

4.2.4 Au @ $\mathrm{TiO}_{2}$ Nanocomposites by X-ray Radiolysis. .................................................... 51

4.2.5 Characterization of $\mathrm{Au} @ \mathrm{TiO}_{2}$ Nanocomposites..................................................... 52

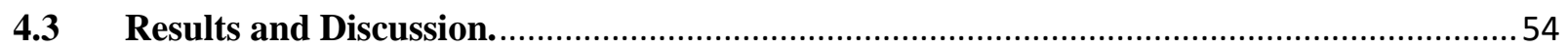

4.3.1 Crystal Structure of $\mathbf{A u} @ \mathbf{T i O}_{2}$ Nanocomposites..................................................... 54

4.3.2 Morphology of $\mathrm{Au} @ \mathrm{TiO}_{2}$ nanocomposites. ................................................................55

4.3.3 Surface chemistry of $\mathrm{Au}_{0} @ \mathrm{TiO}_{2}$ nanocomposites.....................................................65 
Chapter 5: Comparison of X-ray Radiolytic synthesis with traditional Deposition-precipitation

method

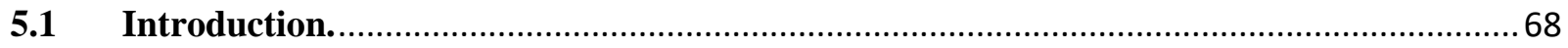

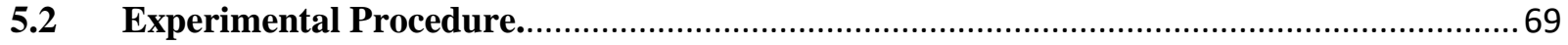

5.2.1 Deposition-precipitation of $\mathrm{Au} @ \mathrm{TiO}_{2}$ Nanocomposites............................................69

5.2.2 Characterization of $\mathrm{Au} @ \mathrm{TiO}_{2}$ Nanocomposites synthesized by Deposition-

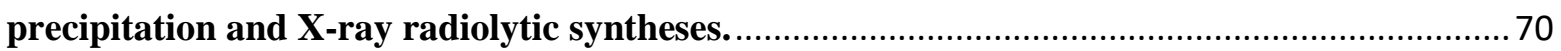

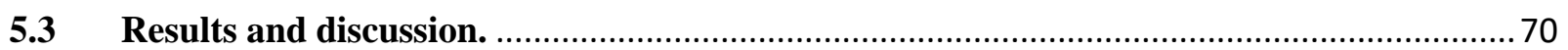

5.3.1 Crystalline structure of $\mathrm{Au} @ \mathrm{TiO}_{2}$ by Deposition-precipitation..................................70

5.3.2 Morphology of $\mathrm{Au} @ \mathrm{TiO}_{2}$ synthesized by Deposition-precipitation............................ 71

5.3.3 Loading comparison of $\mathrm{Au} @ \mathrm{TiO}_{2}$ synthesized by X-ray radiolysis and the Deposition-

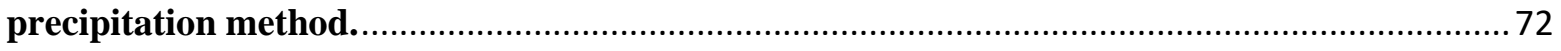

5.3.4 Heating process relevance in $\mathrm{X}$-ray radiolytic synthesis. ...................................... 73

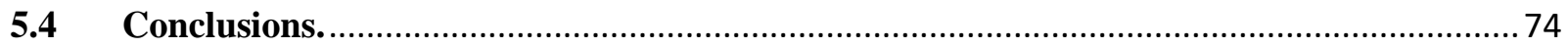

Chapter 6: Au@ $\mathrm{TiO}_{2}$ as Potential radiosensitizers......................................................................... 75

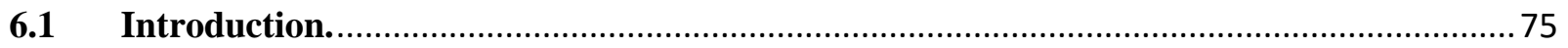

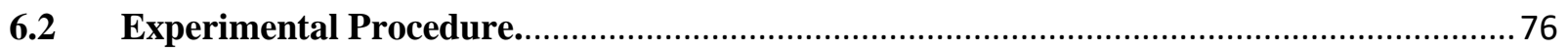

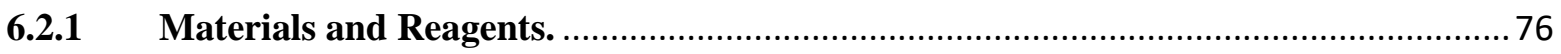

6.2.2 Radiosensitization Analysis of $\mathrm{Au} @ \mathrm{TiO}_{2}$ Nanocomposites using Methylene Blue.....76

6.2.3 Radiosentization of $\mathrm{Au} @ \mathrm{TiO}_{2}$ on Microcystis aeruginosa Cells................................77

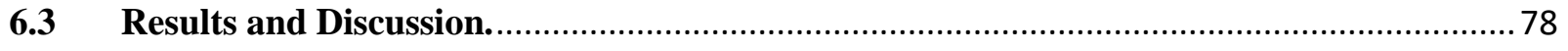

6.3.1 Ligand influence on $\mathrm{Au} @ \mathrm{TiO}_{2}$ radiosensitization.....................................................78

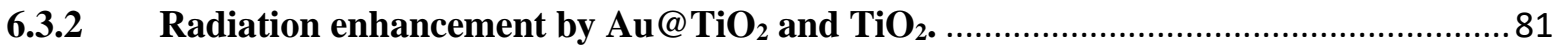

6.3.3 Influence of $\mathrm{X}$-rays energy and concentration in $\mathrm{Au} @ \mathrm{TiO}_{2}$ radiosensitization..........84 
6.3.4 Radiosensitization analysis of $\mathrm{Au} @ \mathrm{TiO}_{2}$ Nanocomposites with different particle size support. 85

6.3.5 Effect of Au loading in MB radiosensitization. 86

6.3.6 Radiosensitization of $\mathrm{Au} @ \mathrm{TiO}_{2}$ Nanocomposites on $\mathrm{M}$. aeruginosa. .88

6.4 Conclusions. .92

Chapter 7: Comparison of $\mathrm{Au} @ \mathrm{TiO}_{2}$ radiosensitization with other metal oxides systems at the megavoltage range

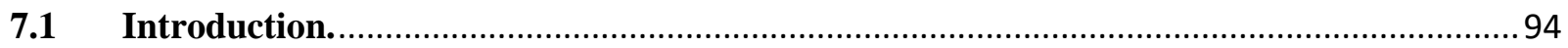

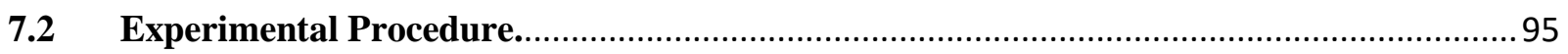

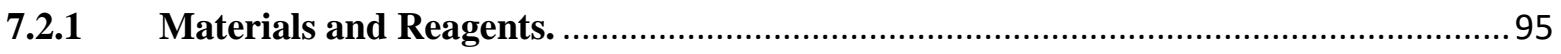

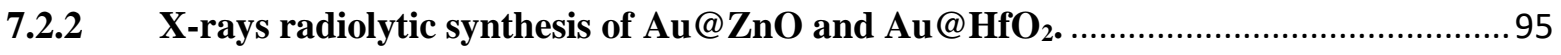

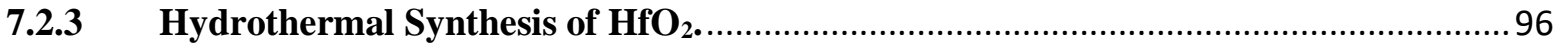

7.2.4 Characterization of metal-oxide nanoparticles. .................................................... 96

7.2.5 Radiosensitization Assessment of metal-oxide semiconductors..............................97

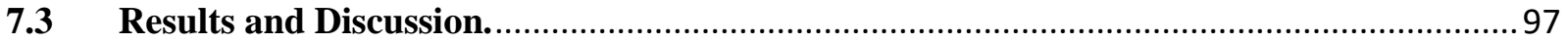

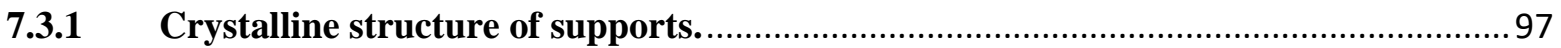

7.3.2 Morphology analysis of metal oxides and Au@oxides.............................................98

7.3.3 Radiosensitization of metal oxides and Au@ oxides................................................... 100

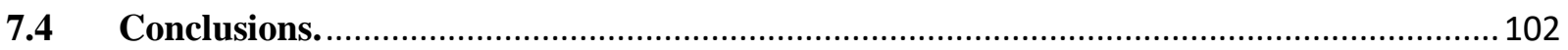

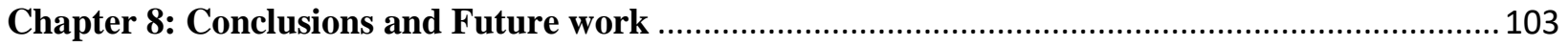

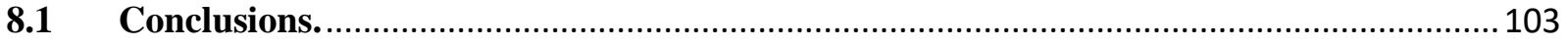

$8.2 \quad$ Future work. 


\section{List of Figures}

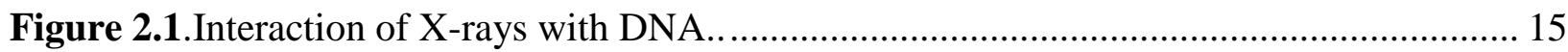

Figure 2.2.Photograph of a LINAC used in external radiation therapy....................................... 17

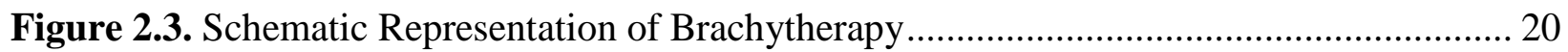

Figure 2.4. a) The mass-energy absorption coefficient for water and $\mathrm{Au}$. b) The ratio between mass energy-absorption coefficients of Au over water is shown as a function of energy............ 30

Figure 2.5. Schematic representation of physical enhancement on High Z nanoparticles. ......... 31

Figure 2.6. Chemical Enhancement Produced by metal oxides nanoparticles. .......................... 32

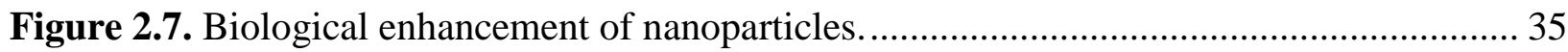

Figure 3.1. Schematic representation of Au supported nanoparticles by the sol-gel method...... 38

Figure 3.2. Schematic representation of the synthesis of Au supported titania by the Deposition-

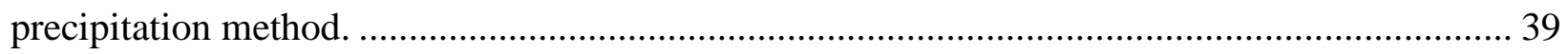

Figure 3.3. Schematic representation of ion reduction by X-ray radiolytic synthesis................. 44

Figure 4.1. Au ion deposition on $\mathrm{TiO}_{2}$ using $\mathrm{NaOH}$ and Urea................................................ 51

Figure 4.2. Experimental Procedure of X-ray radiolytic synthesis of $\mathrm{Au} @ \mathrm{TiO}_{2}$ nanocomposites.

Figure 4.3. Sample Preparation of the different Characterization techniques. ............................ 54

Figure 4.4. XRD Pattern of $\mathrm{Au} @ \mathrm{TiO}_{2}$ nanocomposites synthesized with either $\mathrm{NaOH}$ or urea as ligands and different support sizes at an absorbed dose of $7260 \mathrm{~Gy}$. 55

Figure 4.5. TEMs of $\mathrm{TiO}_{2}$ with a particle size distribution of a) $21.6 \mathrm{~nm}$ and b) $6.5 \mathrm{~nm}$. These images were taken prior to irradiation in order to ensure the integrity of the supports before

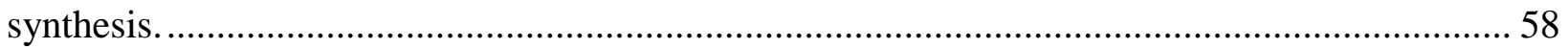


Figure 4.6. TEMs of $\mathrm{Au} @ \mathrm{TiO}_{2}{ }^{(21.6 \mathrm{~nm})-\mathrm{NaOH}}$ nanocomposites with an absorbed dose of a) 120 b) 240 and c) $480 \mathrm{~Gy}$ 58

Figure 4.7. TEMs of $\mathrm{Au} @ \mathrm{TiO}_{2}$ nanocomposites at an absorbed dose of 720 Gy for a) $\mathrm{Au} @ \mathrm{TiO}_{2}{ }^{(21.6 \mathrm{~nm})-\mathrm{NaOH}}$, and b) $\mathrm{Au} @ \mathrm{TiO}_{2}{ }^{(21.6 n m)-u r e a}$, with inset of SAED pattern showing $\mathrm{Au}$ reflections by white circles and anatase by red dashed circles. 60

Figure 4.8. TGA analysis of $\mathrm{Au} @ \mathrm{TiO}_{2}$ fabricated with $\mathrm{NaOH}$ and Urea. 62 Figure 4.9. a) UV Vis of $\mathrm{Au} @ \mathrm{TiO}_{2}{ }^{(6.5 \mathrm{~nm})-\mathrm{NaOH}}$ nanocomposites and the evolution of $\mathrm{Au}$ plasmon resonance at a dose rate of $127 \mathrm{~Gy} / \mathrm{min}$ b) $\mathrm{STEM}$ of $\mathrm{Au} @ \mathrm{TiO}_{2}{ }^{(6.5 \mathrm{~nm})-\mathrm{NaOH}}$ at 60 minutes of irradiation time (7260 Gy) c) UV Vis of $\mathrm{Au} @ \mathrm{TiO}_{2}{ }^{(21.6 n m)-\mathrm{NaOH}}$ nanocomposites and the evolution of Au plasmon resonance up to 90 minutes of irradiation.

Figure 4.10. TEMs of nanocomposites with their respective Au particle size distribution with an absorbed dose of $7260 \mathrm{~Gy}$ for a) $\mathrm{Au} @ \mathrm{TiO}_{2}{ }^{(21.6 n m)-u r e a}$ and b) $\mathrm{Au} @ \mathrm{TiO}_{2}{ }^{(6.5 \mathrm{~nm}) \text {-urea }}$. 64

Figure 4.11. HR-XPS spectra of $\mathrm{Au} @ \mathrm{TiO}_{2}{ }^{(21.6 \mathrm{~nm})-\mathrm{NaOH}}$ nanocomposites using an absorbed dose of 7260 Gy a) Au4f and b) O1s. 65

Figure 5.1. Experimental Procedure of $\mathrm{Au} @ \mathrm{TiO}_{2}$ nanocomposites fabricated by the Depositionprecipitation method. 69

Figure 5.2. XRD of $\mathrm{Au} @ \mathrm{TiO}_{2}$ nanocomposites using radiolytic synthesis and Depositionprecipitation method. 71

Figure 5.3. Au@ $\mathrm{TiO}_{2}$ nanocomposites fabricated with the Deposition-precipitation method onto a) $21.6 \mathrm{~nm}$ supports b) $6.5 \mathrm{~nm}$ supports. 72

Figure 5.4. a) TEM of $\mathrm{Au} @ \mathrm{TiO}_{2}$ synthesized in the absence of the heating process. b) $\mathrm{UV}-\mathrm{Vis}$ of $\mathrm{Au} @ \mathrm{TiO}_{2}$ synthesized in the presence and in the absence of heating. 74 
Figure 6.1. Schematic representation of $\mathrm{Au} @ \mathrm{TiO}_{2}$ irradiation on $\mathrm{MB}$ solution and their subsequent degradation measurement. 77

Figure 6.2. Irradiation set up of $\mathrm{M}$. aeruginosa cells containing $\mathrm{TiO}_{2}$ and $\mathrm{Au} @ \mathrm{TiO}_{2}$ nanoparticles.

Figure 6.3. Kinetics of MB decrease under X-rays in the presence of $\mathrm{Au} @ \mathrm{TiO}_{2}{ }^{(6.5 \mathrm{~nm})-\mathrm{NaOH}-10 \%}$ and $\mathrm{Au} @ \mathrm{TiO}_{2}{ }^{(6.5 \mathrm{~nm})-\mathrm{Urea}-10 \%}$ at a concentration of $0.2 \mathrm{mg} / \mathrm{ml}$. Irradiation carried out at $50 \mathrm{kV}$ and a dose rate of $35 \mathrm{~Gy} / \mathrm{min}$. 80

Figure 6.4. $\mathrm{C} 1 \mathrm{~s}$ HR-XPS Analysis of a) $\mathrm{Au} @ \mathrm{TiO}_{2}{ }^{(21.6 \mathrm{~nm})-\mathrm{Urea}-10 \%}$ b) $\mathrm{Au} @ \mathrm{TiO}_{2}{ }^{(21.6 \mathrm{~nm})-\mathrm{NaOH}-10 \%} 80$ Figure 6.5. Reaction rate of $\mathrm{MB}$ using $\mathrm{X}$-rays in the presence of $\mathrm{TiO}_{2}{ }^{(6.5 \mathrm{~nm})}$ at different nanomaterial concentration. Irradiation carried out at $50 \mathrm{KV}$ and $35 \mathrm{~Gy} / \mathrm{min}$. 81

Figure 6.6. Reaction rate of $\mathrm{MB}$ using X-rays in the presence of $\mathrm{TiO}_{2}{ }^{(6.5 \mathrm{~nm})}$ and $\mathrm{Au} @ \mathrm{TiO}_{2}{ }^{(6.5 \mathrm{~nm}) \text { - }}$ $\mathrm{NaOH}-10 \%$ at a concentration of $1 \mathrm{mg} / \mathrm{ml}$. Irradiation carried out at $50 \mathrm{kV}$ and a dose rate of 35 Gy/min. 83

Figure 6.7. $\mathrm{MB}$ degradation in the presence of $\mathrm{Au} @ \mathrm{TiO}_{2}{ }^{(6.5 \mathrm{~nm})-\mathrm{NaOH}-10 \%}$ nanocomposites at concentration of a) $0.2 \mathrm{mg} / \mathrm{ml}$ nanocomposites concentration b) $1 \mathrm{mg} / \mathrm{ml}$. Beam voltages: 50 and $225 \mathrm{kV}$ using a dose rate of $35 \mathrm{~Gy} / \mathrm{min}$. 85

Figure 6.8. $\mathrm{MB}$ degradation in the presence of $\mathrm{Au} @ \mathrm{TiO}_{2}{ }^{(6.5 \mathrm{~nm})-\mathrm{NaOH}-10 \%}$ and $\mathrm{Au} @ \mathrm{TiO}_{2}{ }^{(21.6 \mathrm{~nm})-\mathrm{NaOH}-}$ $10 \%$ at a concentration of $0.2 \mathrm{mg} / \mathrm{ml}$. Irradiation carried out using a dose rate of $3 \mathrm{~Gy} / \mathrm{min}$ and a voltage of $225 \mathrm{kV}$ 86

Figure 6.9. $\mathrm{MB}$ degradation in the presence of $\mathrm{Au} @ \mathrm{TiO}_{2}{ }^{(21.6 \mathrm{~nm})-\mathrm{NaOH}-10 \%}$ and $\mathrm{Au} @ \mathrm{TiO}_{2}{ }^{(21.6 \mathrm{~nm})-}$ $\mathrm{NaOH}-2 \%$ at a concentration of $0.2 \mathrm{mg} / \mathrm{ml}$. Irradiation carried out using a dose rate of $3 \mathrm{~Gy} / \mathrm{min}$ and a voltage of $225 \mathrm{kV}$ 87 
Figure 6.10. SEM images showing the interaction of $\mathrm{TiO}_{2}$ and $\mathrm{Au} @ \mathrm{TiO}_{2}$ with $\mathrm{M}$. aeruginosa. a) cells prior irradiation b) cells damage after irradiation c) cells interaction with $\mathrm{TiO}_{2}$ d) cells irradiated in the presence of $\mathrm{TiO}_{2}$ e) cells interaction with $\mathrm{Au} @ \mathrm{TiO}_{2} \mathbf{f}$ ) cells irradiated in the presence of $\mathrm{Au} @ \mathrm{TiO}_{2}$. Cells irradiated with a dose of 6 kGy 90

Figure 6.11. UV-Vis spectra of $M$. aeruginosa a) Nonirradiated cells b) Irradiated cells c) Irradiated cells in the presence of $\mathrm{TiO}_{2}$ d) irradiated cells in the presence of $\mathrm{Au} @ \mathrm{TiO}_{2}$. Measurements were taken 3 days after irradiation at a dose of $6 \mathrm{kGy}$................................ 91

Figure 7.1. XRD Patterns of metal oxides and Au@ oxides. .................................................. 98

Figure 7.2. TEMs of metal oxides for a) $\mathrm{ZnO}$ b) $\mathrm{Au} @ \mathrm{ZnO}$ c) $\mathrm{HfO}_{2}{ }^{75 \mathrm{~nm}}$ d) $\mathrm{Au} @ \mathrm{HfO}_{2}{ }^{75} \mathrm{~nm}$ e) .. 99 Figure 7.3. Increase of $\mathrm{MB}$ reaction rate constant in the presence of metal oxides and Au@metal oxides. 101

Figure 8.1. Degradation of $\mathrm{MB}$ after 10 cycles using $\left.\mathrm{Au} @ \mathrm{TiO}_{2}{ }^{(6.5} \mathrm{nm}\right)-\mathrm{NaOH}$ nanocomposites. Irradiation carried out at $50 \mathrm{KV}$ and $35 \mathrm{~Gy} / \mathrm{min}$ 109 


\section{List of Tables}

Table 2.1. Reactive species yield in pulse radiolysis and steady-state radiation [116].............. 33

Table 3.1.The concentration of reactive species generated during radiolysis of water regarding the

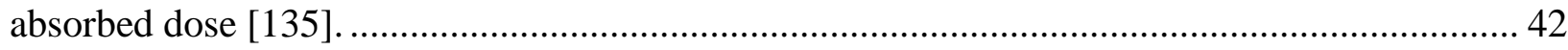

Table 3.2. Standard reduction potential values of some metals [136] ..................................... 46

Table 4.1. ICP OES of $\mathrm{Au} @ \mathrm{TiO}_{2}$ loading and decoration at a total absorbed dose of $7260 \mathrm{~Gy}$.

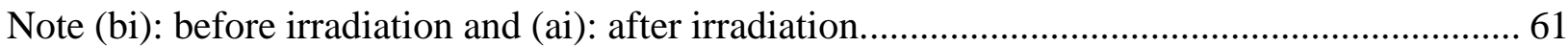

Table 5.1. Loading of $\mathrm{Au}$ supported $\mathrm{TiO}_{2}$ by $\mathrm{X}$-ray radiolytic synthesis and the Depositionprecipitation method. 73

Table 6.1. Summary of $\mathrm{MB}$ reaction rates in the presence of $\mathrm{TiO}_{2}$ and $\mathrm{Au} @ \mathrm{TiO}_{2}$ nanocomposites. 88

Table 7.1. Summary of MB degradation in the presence of different metal oxides and Au supported oxides. 101 


\title{
Abstract \\ Au@ TiO $_{2}$ NANOCOMPOSITES SYNTHESIZED BY X-RAY RADIOLYSIS AS POTENTIAL RADIOSENSITIZERS
}

\author{
By Maria C. Molina Higgins
}

A dissertation submitted in partial fulfillment of the requirements for the degree of Doctor of Philosophy at Virginia Commonwealth University.

Virginia Commonwealth University, 2019

Director: Dr. Jessika Rojas

Assistant Professor, Department of Mechanical and Nuclear Engineering.

Radiosensitization is a novel targeted therapy strategy where chemical compounds are being explored to enhance the sensitivity of the tissue to the effects of ionizing radiation. Among the different radiosensitizers alternatives, nanomaterials have shown promising results by enhancing tumor injury through the production of free radicals and reactive oxygen species (ROS). In this work, Gold-supported titania $\left(\mathrm{Au} @ \mathrm{TiO}_{2}\right)$ nanocomposites were synthesized through an innovative strategy using X-ray irradiation, and their potential as radiosensitizers was investigated. Radiosensitization of $\mathrm{Au} @ \mathrm{TiO}_{2}$ nanocomposites was assessed by monitoring the decomposition of Methylene Blue (MB) under X-ray irradiation in the presence of the nanomaterial. Radiosensitization of $\mathrm{Au} @ \mathrm{TiO}_{2}$ was thoroughly investigated as a function of parameters such as $\mathrm{Au}$ loading, $\mathrm{TiO}_{2}$ particle size, nanomaterial concentration, different irradiation voltages, and dose rates. Results showed that the presence of $\mathrm{Au} @ \mathrm{TiO}_{2}$ increases significantly the absorbed dose, thus enhancing $\mathrm{MB}$ decomposition. The mechanism behind $\mathrm{Au} @ \mathrm{TiO}_{2}$ radiosensitization relies on their interaction with $\mathrm{X}$-rays. $\mathrm{TiO}_{2}$ produces reactive $\mathrm{ROS}$ whereas $\mathrm{Au}$ leads to the generation of photoelectrons and Auger electrons upon exposure to X-rays. These species lead to an enhanced degradation rate of the dye, a feature that could translate to cancerous cells damage with minimal 
side effects. The radiosensitization effect of $\mathrm{Au} @ \mathrm{TiO}_{2}$ nanocomposites was also tested in biological settings using Microcystis Aeruginosa cells. The results showed an increase in cell damage when irradiated in the presence of $\mathrm{Au} @ \mathrm{TiO}_{2}$ nanocomposites. $\mathrm{Au} @ \mathrm{TiO}_{2}$ nanocomposites were fabricated using X-ray radiolytic synthesis, a method that diverges from conventional fabrication processes and leads to negligible by-product formation, an important feature for medical and catalytic applications. In this work, $\mathrm{Au}$ nanoparticles are supported on $\mathrm{TiO}_{2}$ with a mean particle size of either $6.5 \mathrm{~nm}$ or $21.6 \mathrm{~nm}$, using different ligands such as $\mathrm{NaOH}$ or urea, and under different absorbed doses to determine the effects of these parameters on the nanomaterials' characteristics. Overall, $\mathrm{Au} @ \mathrm{TiO}_{2}$ synthesized by X-rays showed remarkable promise as radiosensitizers, a concept relevant to a number of medical, biological and environmental applications. 


\section{Chapter 1: Motivation and Research Objectives}

\subsection{Introduction.}

Radiation therapy is a minimally invasive procedure for cancer treatment used to prevent progression of the disease and destroy abnormal tissue. Nowadays, half of the patients diagnosed with cancer will benefit from the use of ionizing radiation for curative and/or palliative purposes [1]. Nonetheless, radiation therapy may lead to side effects caused by damage to healthy tissue and cells becoming resistant to radiation [2]. Therefore, current efforts toward new strategies to increase the efficacy of radiotherapy are directed to personalized targeted treatments, aiming to maintain a good quality of life of the patients affected by cancer [2-4]. Among the different strategies, radiosensitization relies on the use of chemical compounds to increase the sensitivity of tumors to the effects of ionizing radiation [5]. The compounds, commonly known to as radiosensitizers, are classified based on their chemical structure and they are categorized into small-molecule, macromolecules, and nanostructures [5]. Small-molecules radiosensitizers are simple molecules such as oxygen $\left(\mathrm{O}_{2}\right)$ that rely on electron affinity to enhance cell damage in the presence of radiation, the use of small-molecules as radiosensitizers is particularly beneficial in hypoxia conditions [7]. Macromolecules are long-chain compounds such as proteins and peptides that bind to the DNA, they contain agents that will lead to an increase in the radiosensitivity of cells [5]. Finally, radiosensitizers research on nanomaterials has evidenced great promise, since certain materials can absorb and amplify the radiation delivered during treatment [5-7]. Inorganic nanomaterials made of elements with a high atomic number such as gold $(\mathrm{Au})$ or hafnium $(\mathrm{Hf})$ have the ability to produce enhanced cell damage when combined with traditional forms of radiation therapy $[4,6,7]$. Some of the nanomaterials that have been investigated as radiosensitizers are: lanthanide-based nanoparticles, silicon [8], titanium [9], zinc and hafnium oxides nanostructures (i.e. nanoparticles and nanotubes) 
[10,11], quantum dots [12], superparamagnetic oxides [4], silica, and Au nanoparticles [13-15]. Among the different radiosensitizers alternatives, Au nanoparticles have been extensively studied because of their anti-cancer potential. Au nanoparticles have shown biocompatibility both in-vivo and in-vitro experiments, and they offer the possibility of different synthesis processes that result in a variety of sizes and shapes. Additionally, Au has been successfully used in combination with bioconjugation techniques, allowing for the targeting of tumors on specific sites of the human body $[3,4,13-16]$.

The use of radiation as a therapeutic tool for cancer treatment relies on its mechanism of interaction with tissue. Ionizing radiation interacts with the water in the cells through a mechanism termed radiolysis, a process that generates reactive oxygen species (ROS) such as hydroxyl radicals $\left(\mathrm{OH}^{*}\right)$, superoxide radicals $\left(\mathrm{O}_{2}^{-*}\right)$, and hydrogen peroxide $\left(\mathrm{H}_{2} \mathrm{O}_{2}\right)$. These species ultimately lead to cell damage. The use of $\mathrm{Au}$ as radiosensitizer has been proposed due to its higher photoelectric absorption cross section when compared to soft tissue and water [17]. The interaction of $\mathrm{Au}$ nanoparticles with X-rays results in the emission of secondary radiation such as photoelectrons, Compton and Auger electrons, and fluorescence photons emissions with different energies and penetration depths, causing an increment in the dose delivered to the tissue $[15,18]$. The energetic particles, produced by the interaction of Au with X-rays, cause ionization and excitation of molecules as they travel, followed by the generation of ROS known to cause cellular damage $[3,16,19,20]$. Specifically, ROS can produce changes in cell cycle processes that cause severe damage in the DNA structure [3,21]. On the other hand, nanomaterials composed of oxides such as titanium dioxide $\left(\mathrm{TiO}_{2}\right)$, also known as titania, have been successfully utilized in photodynamic therapy, a treatment that uses a photosensitizer in combination with a source of light with a specific wavelength. The interaction of the photosensitizer with light will produce ROS ultimately causing 
cell death [22]. However, this treatment has been used only to treat superficial tumors due to the low penetration depth of visible and UV-light $[22,23]$. The use of $\mathrm{TiO}_{2}$ to treat internal tumors can be extended by combining it with highly penetrating radiation such as X-rays [9]. The interaction of $\mathrm{TiO}_{2}$ with X-rays photons leads to the generation of electrons-holes pairs or carriers that interact with nearby water molecules producing ROS such as $\mathrm{OH}^{-}, \mathrm{O}_{2}^{--}$, and $\mathrm{H}^{+}$. Nonetheless, $\mathrm{TiO}_{2}$ has shown a high rate of electron-hole recombination, reducing the ROS production of the metal-oxide at its surface. A strategy used to minimize the recombination of carriers is the surface modification of $\mathrm{TiO}_{2}$ with $\mathrm{Au}$ [24-26]. Au deposited on the $\mathrm{TiO}_{2}$ surface reduces the band gap energy barrier and the recombination rate of electron-hole pairs, leading to an overall enhancement of ROS production [21]. Thus, in this work we produced, characterized, and evaluated gold-titania $\left(\mathrm{Au} @ \mathrm{TiO}_{2}\right)$ nanocomposites for its use as a potential radiosensitizer, by combining the synergistic effect of ROS produced by $\mathrm{TiO}_{2}$ and the photo-emissions by Au upon X-ray irradiation.

$\mathrm{Au} @ \mathrm{TiO}_{2}$ nanocomposites have been synthesized using traditional wet-chemical routes such as deposition-precipitation, hydrothermal and impregnation syntheses. Deposition-precipitation is one of the most widely reported methods to synthesize nanomaterials, the formation of Au nanoparticles is induced through a calcination process. In this method, the Au ions are co-precipitated onto the titania support through a heating process that results in Au complexes in the form of hydroxides, carbonates and citrates depending on the type of ligand present (i.e. $\mathrm{NaOH}$ or urea), followed by calcination processes at high temperature for several hours [29]. On the other hand, hydrothermal syntheses reduce metal cations in solution using elevated temperatures and pressures, producing a nanocomposite with uniform disperse nanoparticles on supports [27,28]. Traditional wet-chemical routes, often involve the use of chemical reducing agents in a high volume, since they rely on the chemical reduction of metal salt precursors in solution to produce nanoparticles [27]. These reducing 
agents could represent a hazard for nanocomposites intended for medical applications. Although wet-chemical routes have been extensively studied [27,29], these methods show some drawbacks such as slow reaction rates, which lead to nanoparticles with a broad size distribution [30]. Other methods such as deposition-precipitation and impregnation that use calcination processes are known to precipitate undesired species onto the nanoparticles, compromising the surface chemistry of the nanocomposite. Thus, this leads to poor performance in catalytic and medical applications where nanocomposites surface chemistry is highly important [27]. Other methods to synthesize $\mathrm{Au} @ \mathrm{TiO}_{2}$ are electro-chemical and sonochemical syntheses, laser ablation and thermal decomposition routes [31-34].

Nowadays, research efforts directed towards novel synthesis methods which improve nanoparticle size control, particle size distribution and morphology are vital for the advancement of the different fields of nanotechnology. Methods that do not require high temperature or pressures, coupled with low or negligible by-product formation while maintaining feasibility, scalability and reproducibility qualities are desirable [35,36]. Radiolytic synthesis of metallic nanoparticles has become a successful synthesis method for producing nanocomposites with excellent nanoparticle dispersion onto supports, while using non-toxic solvents, such as water and alcohols, leading to pure nanocomposites in a synthesis method that does not produce hazardous waste [37-39]. Radiolytic synthesis of metal nanoparticles begins when an aqueous solution containing a metallic precursor is irradiated with high energy particles such as X-rays. This leads to the production of reactive species such as hydrated electrons $\left(\mathrm{e}^{-} \mathrm{aq}\right)$ and free radicals, such as hydrogen radicals $(\mathrm{H} \bullet)$ and $\mathrm{OH} \bullet$ Reducing species such as $\mathrm{e}_{\text {aq }}^{-}$interact with the metal ions reducing their oxidation states. The presence of secondary alcohols such as isopropanol will act as an oxidant scavenger, by transforming oxidizing species such as $\mathrm{OH} \bullet$ into new reducing species that will further reduce the metal ions into atoms 
solution. These metals atoms will form nucleation centers, coalesce and grow into nanoparticles [40]. Important nanoparticle features such as morphology, particle size, particle size distribution, and synthesis yield, strongly depend on the radiation used for the manufacturing. Currently, nanoparticles have been produced using different radiation sources such as electrons, protons, heavy ions, $\gamma$-rays and X-rays [40]. An important parameter known as the linear energy transfer (LET) varies with the type of radiation used in the synthesis and plays an important role regarding the number of reactive species produced in solution and their spatial distribution, which influences nanoparticle nucleation and growth processes[40]. Beams composed of high energy electrons, Xrays and $\gamma$-rays are low LET radiation that leads to a high amount of reducing species per unit energy deposited per unit distance and produce small particles with a narrow particle size distributions [4043]. When compared to $\gamma$-rays, $X$-rays could represent a better alternative for radiolytic syntheses $[42,44]$, since $\gamma$-ray-based fabrication methods hold constraints related to training and restrictions regarding radioactive source licensing [35,45]. X-ray devices, on the other hand, can be found in several medical and research institutions and the radiation source can be turned on and off at any time as needed. Furthermore, X-ray devices can facilitate in-situ characterization during fabrication [45].

In view of $\mathrm{Au} @ \mathrm{TiO}_{2}$ as potential radiosensitizers and the capability of radiolytic synthesis to produce pure nanomaterials, in this work we synthesized $\mathrm{Au} @ \mathrm{TiO}_{2}$ radiosensitizers using X-ray radiolysis performed at absorbed doses from 120 to $7260 \mathrm{~Gy}$, in order to test the influence of the absorbed dose on $\mathrm{Au}$ nanoparticle size, particle size distribution and loading onto the $\mathrm{TiO}_{2}$ support. Moreover, the nanocomposite was fabricated using ligands such as sodium hydroxide $(\mathrm{NaOH})$ and urea. The ligand is a chemical agent that allows for electrostatic interactions between the $\mathrm{TiO}_{2}$ and Au-complexes in solution controlling the nucleation, growth, and binding of Au nanoparticles onto 
the titania surface [46]. Au@ $\mathrm{TiO}_{2}$ nanocomposites as radiosensitizers were evaluated through the decomposition of methylene blue (MB) and their effect on Microcystis Aeruginosa (M. Aeruginosa) cells under X-ray irradiation. M. Aeruginosa cells are unicellular cyanobacteria damaging to water fauna and toxic to humans [47]. These cells were chosen in order to test the radiosensitization effects of the nanocomposites in a biological scenario while showing the possibility of using $\mathrm{Au} @ \mathrm{TiO}_{2}$ for bacteria removal from water. Our results showed that the presence of $\mathrm{Au} @ \mathrm{TiO}_{2}$ nanocomposites in solution increased the reaction rate of $\mathrm{MB}$ and cell damage by augmenting the absorbed dose within the media at all experimental conditions. Thus, the results presented throughout this dissertation have the potential to enable the use of $\mathrm{Au} @ \mathrm{TiO}_{2}$ nanocomposites for future in-vitro and in-vivo and applications.

\subsection{Motivation.}

This research project has as an objective to develop an innovative synthesis route to produce $\mathrm{Au} @ \mathrm{TiO}_{2}$ nanocomposites and evaluate their dose enhancement response to X-rays, in order to enable their use as a potential radiosensitizer. This project is motivated by the need for systematic studies in techniques that potentially improve radiation therapy [7]. Even though radiotherapy is an effective method to treat cancer, the side effects of radiotherapy include skin erythema, fatigue, nausea, tooth decay, among others [48]. Thus, new approaches that improve the therapeutic effectiveness will have a beneficial impact in both physiological and physical wellbeing of the patients affected by cancer. Radiosensitization is a method that allows for a targeted therapy treatment, diminishes cell resistance to radiation and it may produce faster results than conventional radiation therapy [4].

For nanomaterials intended for medical applications, it is important to select a fabrication method that does not compromise the surface chemistry of the nanoparticles. Radiolytic synthesis is one of 
these methods since it has demonstrated it can produce well-dispersed metallic nanoparticles onto different types of supports such as carbon nanotubes, metal oxides, and polymers without the need of harsh reducing agents, high temperatures and elevated pressures [37,49]. X-ray radiolytic synthesis allows control over the final particle size of the nanomaterial by tuning parameters such as the absorbed dose used throughout the synthesis. Furthermore, X-ray irradiation is a mature technology that has been extensively used in various applications such as food irradiation, sterilization of medical instruments and cosmetics, among others [35]. Due to the fact that X-raybased synthesis is an unexplored methodology, its understanding is still limited. In this project, metal oxide supports such as $\mathrm{TiO}_{2}, \mathrm{ZnO}$, and $\mathrm{HfO}_{2}$ were decorated with Au nanoparticles using multiple ligands and synthesis conditions of absorbed dose to provide insight into the radiation chemistry of Au nanoparticle formation onto the support surface. Au@oxides nanocomposites have been widely studied by multiple chemical approaches using different reducing agents in order to obtain different Au loading and particle size distribution, therefore an exploration of different parameters in X-rays radiolytic synthesis is vital to producing nanomaterials able to compete with those developed by traditional fabrication methods.

\subsection{Research Objectives.}

Objective 1: Produce gold nanoparticles supported on titania using X-ray radiolytic synthesis.

The first stage of this research aimed to explore X-ray radiolytic synthesis as a clean alternative to fabricate supported Au nanoparticles. In the clinical field, nanomaterials have become an innovative alternative for early diagnosis and treatment of a significant number of medical conditions. Nanoparticles can be utilized as contrast agents for imaging purposes, carriers for drug delivery, and radiosensitizers [50]. Particularly, for radiosensitization, the use of nanoparticles composed of 
biocompatible noble metals is becoming an attractive approach for enhancing the radiation dose delivered to tissue during radiation therapy.

Radiosensitization by nanoparticles often depends on their particle size, morphology and synthesis route. The most commonly used methods to synthesize metallic nanoparticles involve the chemical reduction of a metal precursor [51]. Radiation synthesis is a novel method where chemical reducing agents are not needed, thus radiolytic synthesis does not generate chemical waste that may affect the nanoparticles' properties [35]. Furthermore, this technique is designed to work at room temperature and under atmospheric pressures [38,39]. Nonetheless, the most outstanding feature of radiation synthesis is the possibility to control the particle size and distribution by tuning the dose rate delivered during synthesis [52,53]. This stage investigated Au nanoparticle formation, growth, and morphology by varying different synthesis parameters such as absorbed doses, ligands, $\mathrm{TiO}_{2}$ particle size, and Au nanoparticle loading in order to find the optimal combination of parameters that enable the use of $\mathrm{Au} @ \mathrm{TiO}_{2} \mathrm{NCs}$ as radiosensitizers in future in-vivo and in-vitro experiments. The tasks followed to complete objective 1 are listed below.

\section{Tasks:}

1.1. Investigate the effect of the absorbed dose and dose rate on Au nanoparticle formation, morphology, and loading onto $\mathrm{TiO}_{2}$ supports.

1.2. Evaluate the interaction mechanism(s) of Au nanoparticles with $\mathrm{TiO}_{2}$ by using different ligands such as $\mathrm{NaOH}$ and Urea.

1.3. Compare Au nanoparticle particle formation on $\mathrm{TiO}_{2}$ by traditional chemical methods such as deposition-precipitation with that of X-rays radiolytic synthesis. 


\section{Objective 2: Evaluate the radiosensitization effect of $\mathrm{Au}_{0} @ \mathrm{TiO}_{2}$ nanocomposites through the decomposition of MB under X-ray irradiation.}

The degradation of $\mathrm{MB}$ in the presence of nanomaterials as a chemical dosimeter to test radiosensitization has been used by researchers in the past [54]. Even though MB degradation by nanoparticles is an indirect method to measure radiosensitization, it allows exploring the radiosensitization mechanisms of physical and chemical enhancement. Pre-clinical research on targeted therapy strategies such as radiosensitization is of valuable interest, since the use of radiosensitizers may reduce collateral damage to healthy tissue. In this objective, gold supported titania $\left(\mathrm{Au} @ \mathrm{TiO}_{2}\right)$ nanocomposites were evaluated for their use as potential radiosensitizers by using $(\mathrm{MB})$ as a chemical probe. The interaction of $\mathrm{X}$-rays with $\mathrm{TiO}_{2}$ generates the emission of reactive oxygen species (ROS), whereas Au interaction with X-rays leads to the generation of photoelectrons and Auger electrons; these emissions cause MB degradation.

This objective is motivated by the need for new methodologies, such as radiosensitization, that could potentially improve radiation therapy. Therefore, $\mathrm{Au} @ \mathrm{TiO}_{2}$ radiosensitization was investigated by varying parameters such as $\mathrm{Au}$ loading, $\mathrm{TiO}_{2}$ particle size, nanomaterial concentration, irradiation voltages, and dose rates. $\mathrm{Au} @ \mathrm{TiO}_{2}$ were fabricated using X-rays, a methodology that departs from traditional synthesis and has demonstrated to produce well-dispersed metallic nanoparticles onto different types of supports. Well established synthesis procedures, that resulted from the successful completion of objective one, were implemented in this stage.

\section{Tasks:}

2.1 Evaluate radiosensitization on bare $\mathrm{TiO}_{2}$ and $\mathrm{Au} @ \mathrm{TiO}_{2}$ with different support mean particle size and Au nanoparticle loading. 
2.2 Analyze the effect of different X-rays voltages $(50 \mathrm{kV}, 225 \mathrm{kV}$, and $6 \mathrm{MV})$ on the radiosensitization of $\mathrm{Au} @ \mathrm{TiO}_{2}$

2.3 Investigate the radiosensitization of $\mathrm{Au} @ \mathrm{TiO}_{2}$ under different dose rate conditions (3 Gy, $8 \mathrm{~Gy}$, and $35 \mathrm{~Gy})$.

\section{Objective 3: Synthesize other potential nanocomposites such as $\mathrm{Au} @ \mathrm{ZnO}$ and $\mathrm{Au} @ \mathrm{HfO}_{2}$ in order to compare their dose enhancement to $\mathrm{Au} @ \mathrm{TiO}_{2}$ nanocomposites}

This objective focuses on the use of wide bandgap semiconductors such as $\mathrm{ZnO}$ and $\mathrm{HfO}_{2}$ and their evaluation as radiosensitizers using $\mathrm{MB}$ as a chemical dosimeter. Specifically, $\mathrm{ZnO}$ is a semiconductor used in different catalytic applications. $\mathrm{HfO}_{2}$ is a semiconductor composed of high $\mathrm{Z}$ elements and it has been used for electronic and target therapy applications. Since $\mathrm{TiO}_{2}, \mathrm{ZnO}$, and $\mathrm{HfO}_{2}$ are wide bandgap semiconductors deposition of high $\mathrm{Z}$ metals such as $\mathrm{Au}$ are used to reduce the recombination rate of electron-hole pairs, and the bandgap of the semiconductor [39]. Herein, the radiosensitization of the metal oxides was evidenced by monitoring methylene blue (MB) degradation in the presence of the nanomaterial under a LINAC with an energy endpoint of $6 \mathrm{MV}$. Results showed that the addition of metal oxides to $\mathrm{MB}$ accelerates the dye reaction rate by increasing the number of reactive oxygen species in solution.

\section{Tasks:}

3.1 Synthesize $\mathrm{Au}$ supported on $\mathrm{ZnO}$ and $\mathrm{HfO}_{2}$ using $\mathrm{X}$-ray radiolytic synthesis.

3.2. Compare the radiosensitization of $\mathrm{Au}$ supported $\mathrm{ZnO}$ and $\mathrm{HfO}_{2}$ with that of $\mathrm{Au} @ \mathrm{TiO}_{2}$ nanocomposites. 


\section{Objective 4: Investigate the $\mathrm{X}$-rays dose rate enhancement response by $\mathrm{Au} @ \mathrm{TiO}_{2}$ nanocomposites using Microcystis aeruginosa cells.}

$\mathrm{Au} @ \mathrm{TiO}_{2}$ radiosensitization was also tested in a biological environment with $\mathrm{M}$. aeruginosa cells, cyanobacteria toxic to human and wild animals. The aim of this study was to evaluate the damage of $\mathrm{M}$. aeruginosa when exposed to $\mathrm{TiO}_{2}$ and $\mathrm{Au} @ \mathrm{TiO}_{2}$ nanoparticles. Previous experiments are associated with the degradation of $\mathrm{MB}$ in the presence of the nanomaterial, aiming to show the physical and chemical dose enhancement mechanisms of the nanocomposites. These enhancements are associated with the ROS production of $\mathrm{TiO}_{2}$, the photoemissions released by Au upon interaction with X-rays and the overall catalytic activity of the nanomaterial. Experiments with $\mathrm{M}$. aeruginosa on the other hand, show the interaction of radiation with the nanomaterial in a biological scenario, where the ROS and photoemissions interact with the cellular content of the cells causing lipid peroxidation and ultimately cell death. The physical damage caused by the radiosensitizers on the cells was evaluated using Scanning electron microscopy (SEM). Furthermore, chlorophyll concentration was measured before and after irradiation to monitor the damage of $\mathrm{M}$. aeruginosa cells when irradiated in the presence of $\mathrm{TiO}_{2}$ and $\mathrm{Au} @ \mathrm{TiO}_{2}$. Results showed a significant decrease

in the M. aeruginosa cells population when they were exposed to $\mathrm{Au} @ \mathrm{TiO}_{2}$ nanocomposites. This experiment showed promising antibacterial properties of $\mathrm{Au} @ \mathrm{TiO}_{2}$ nanocomposites, this feature could be important in water treatment applications.

\section{Tasks:}

4.1 Characterize physical damage of M. aeruginosa cells in the presence of the radiosensitizers

4.2 Evaluate chlorophyll concentration and M. aeruginosa cell population decrease in the presence of the radiosensitizers. 
The structure of this dissertation is as follows: in chapter 1 the motivation and research objectives behind this work are explained. In chapter 2, a detailed description of radiation therapy, its challenges and, radiosensitization research is presented. Furthermore, this chapter highlights the promise of nanomaterials as radiosensitizers and the different radiation enhancement mechanisms presented by radiosensitizers are illustrated. Chapter 3 is an overview of the different fabrication methods of gold supported on titania synthesis. Herein, the process of radiolytic synthesis of nanomaterials is described in detail and its advantages regarding other fabrication processes are explained. Chapter 4 shows the nucleation and growth of Au nanoparticles supported on $\mathrm{TiO}_{2}$ using $\mathrm{X}$-rays radiolytic synthesis. Different parameters such as absorbed dose, ligands, and support particle size were explored in order to understand the $\mathrm{Au}$ deposition on $\mathrm{TiO}_{2}$ in the presence of X-rays. In chapter 5, the method of X-rays radiolytic synthesis is compared with deposition-precipitation, the traditional methodology to synthesize $\mathrm{Au} @ \mathrm{TiO}_{2}$. Parameters such as the particle size and Au loading are studied with different characterization techniques such as transmission electron microscopy (TEM) and energy-dispersive X-ray spectroscopy. In chapter 6 evaluation of $\mathrm{Au} @ \mathrm{TiO}_{2}$ radiosensitization is carried out using MB and cells of M. Aeruginosa cells. Parameters such as irradiation dose rate, nanomaterial concentration, Au loading, and support particle size were evaluated in order to evidence their effect on the radiosensitizer performance. In chapter 7 a comparison of $\mathrm{Au} @ \mathrm{TiO}_{2}$ radiosensitization with other metal oxides-based nanoparticles such as $\mathrm{ZnO}, \mathrm{Au} @ \mathrm{ZnO}, \mathrm{HfO}_{2}$, and $\mathrm{Au} @ \mathrm{HfO}_{2}$ was made. For this purpose, the nanomaterials were evaluated using MB as a chemical dosimeter and irradiated a medical LINAC with an endpoint energy of 6 $\mathrm{MeV}$. Finally, in chapter 8 the conclusions and the outlook of this project is presented. 


\section{Chapter 2: Radiosensitization Background and Significance}

\subsection{Radiotherapy.}

The main purpose of radiotherapy is to reduce the size of the tumor, by destroying malignant cells with the aid of internal and/or external sources of radiation such as gamma rays, $\mathrm{x}$-rays, proton and electron beams [55-58]. Radiation therapy is a treatment tailored according to the patient's needs, where radiation can be used as the primary treatment method as well as a complementary therapy and for palliative purposes $[59,60]$. The first scenario is when radiation is used as an exclusive method to treat cancer, this procedure is minimally invasive and offers the patient with the option to keep the organ affected by the disease, which is not possible with other invasive techniques such as surgery [60]. On the other hand, complementary radiotherapy is utilized along with another treatment strategy such as surgery or chemotherapy in order to reduce the size of the tumor. Moreover, complementary radiotherapy is a method that often minimizes the probability of cancer relapse [61-63]. Finally, palliative radiotherapy has as an objective to improve or reduce the pain caused by the disease $[64,65]$.

The biological mechanism of radiation therapy is based on the interaction of ionizing radiation with the cells within the tumor by means of direct or indirect action [66,67]. Direct action is associated with the damage that ionizing radiation causes to biomolecules such as protein, lipids, and DNA. Damage caused to DNA molecules is particularly important since DNA is highly susceptible to the effects of radiation. DNA damage can block features such as cell division and proliferation processes, and induce cell necrosis or apoptosis [5,67]. Indirect action, on the other hand, refers to the production of free radicals and ROS [66]. These species are known to have unpaired electrons and they can injure biomolecules through chemical reactions such as hydrogen 
extraction, electron capture, addition, and disproportionation, leading to structural cell damage caused by that the breaking of the single and double strand of DNA $[5,66,67]$.

\subsubsection{Physics behind radiation therapy.}

The interaction of radiation within the human body is affected by many factors such as beam energy, tissue density, composition and patient's distance to the machine [55]. The distribution of radiation in the patient is associated with the dose deposition, where the absorbed dose is the energy deposited in a medium per unit mass as a result of their interaction with ionizing radiation [55]. Most of the patients treated with radiation will undergo therapy with X-rays. When X-rays are used to treat tumors, the photons interact with the atoms in the human body, transferring their energy to their electrons. The damage caused to carcinogenic cells is due to the combined energy deposition of both photons and electrons [55]. At low beam energies ranging from 10-100 keV, the principal mechanism of interaction of radiation with matter is the photoelectric effect $[68,69]$. A process in which electrons, also known as photoelectrons, are ejected from the atoms in the material as a result of a complete energy transfer from the photon. Nonetheless, in conventional radiation therapy, where the mean energy of the photons range from 1 up to the endpoint energy of $20 \mathrm{MeV}$, Compton scattering is the predominant effect [55,68]. In this case, photons interact with the electrons in the atoms, resulting in a recoil electron and a scattered photon [68]. Another interaction phenomenon that occurs during treatment with high energy photons is pair production, a process where a photon with energy above $1.02 \mathrm{MeV}$ interacts with matter and leads to the production of an electron and a positron. When the positron encounters an electron, it annihilates producing two photons with an energy of $0.511 \mathrm{MeV}$. The path and the range of an electron depend on its initial energy and the density of the medium [68]. Figure 2.1 shows the interaction of photons 
with a tissue when using an x-ray beam. In the images, $E_{\gamma 1,2}$ represents the energy of the photons, $E_{k 1,2}$ the kinetic energy of the electrons and $E_{b}$

\section{(a) Photoelectric Effect DNA}
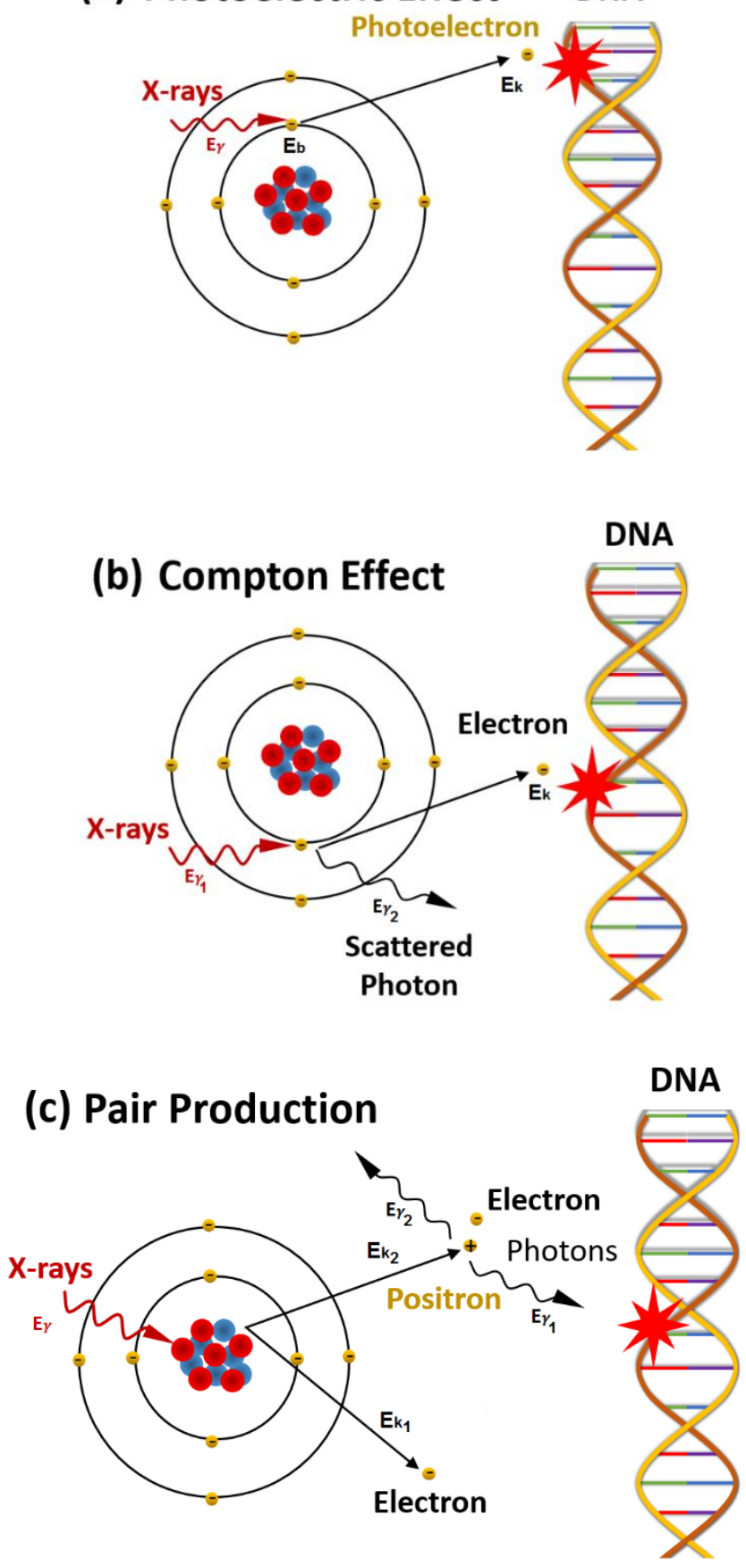

Figure 2.1.Interaction of X-rays with DNA. adapted from [70]. 
As photons interact with tissue, their interaction with water molecules leads to radiolysis. This is a fast process that occurs in a time scale of $10^{-12}$ to $10^{-6}$ seconds [71]. Radiolysis is a mechanism in which water molecules break into reactive species such as $\mathrm{OH} \bullet, \mathrm{H}^{+}$, and $\mathrm{O}_{2}^{-\bullet}$, among others. These species are produced in close proximity to DNA and they have the capability to damage molecules within the cells causing brokerage of single and double strands of DNA [5]. Free radical and ROS have unpaired electrons in the outer shell of their structures, and when they interact with cells, they will try to acquire electrons from nearby molecules, in an attempt to neutralize themselves in a process that could also involve other free radicals [71]. Furthermore, ROS interact with sugar-phosphate bonds compromising the integrity of the cell. The cell damage imparted by ROS strongly correlates with the irradiation particle and its corresponding LET [71]. Beams composed of high LET particles (i.e alpha particles) produce more direct damage than a beam composed of low LET radiation such as X-rays. As the LET increases, more energy is deposited per unit length in the media, producing DNA damage and cell destruction [71].

\subsubsection{External Radiotherapy.}

External radiation therapy is carried out using a radiation-producing device that guides high energy X-rays from outside the body into the tumor site; this irradiator is also known as a linear accelerator (LINAC) and it is shown in Figure 2.2. Clinically, linear accelerators produce photons having energies ranging from 1 to $20 \mathrm{MeV}$, these high energies allow for high photon penetration depth, while controlling adverse effects to the skin, an organ sensitive to the effects of radiation [55]. Nowadays, LINACs are designed to focus the treatment in the specific size and shape of the tumor, for this purpose multileaf collimators are used in a technique known as intensity-modulated radiotherapy (IMRT) [72,73]. The production of high energy photons in a LINAC starts with electron emission from a heated gun filament, the energy of these electrons is gradually increased 
by the use of radio waves [55]. The beam of electrons is accelerated and guided to a bending magnet before it hits a target of a high atomic number such as tungsten $(\mathrm{W})$ or lead $(\mathrm{Pb})[74]$. The X-rays produced by the interaction of electrons with the target are collimated and the radiation intensity is modulated using different types of filters, aiming to produce a beam of uniform intensity [55]. Cobalt-60 irradiators are also widely utilized and currently, health centers benefit from their use. Cobalt irradiators produce photons with a mean energy of $1.25 \mathrm{MeV}$ and do not require complex electronics that often are installed in LINACs [75]. The effectiveness of the treatment using external radiation devices relies heavily on an accurate dose delivery to the affected tissue. The identification of the tumor volume and adjacent areas where the tumor can spread and the study of the volume of interest is made using imaging techniques such as computed tomography (CT), magnetic resonance imaging (MRI) and positron emission tomography (PET) [76].

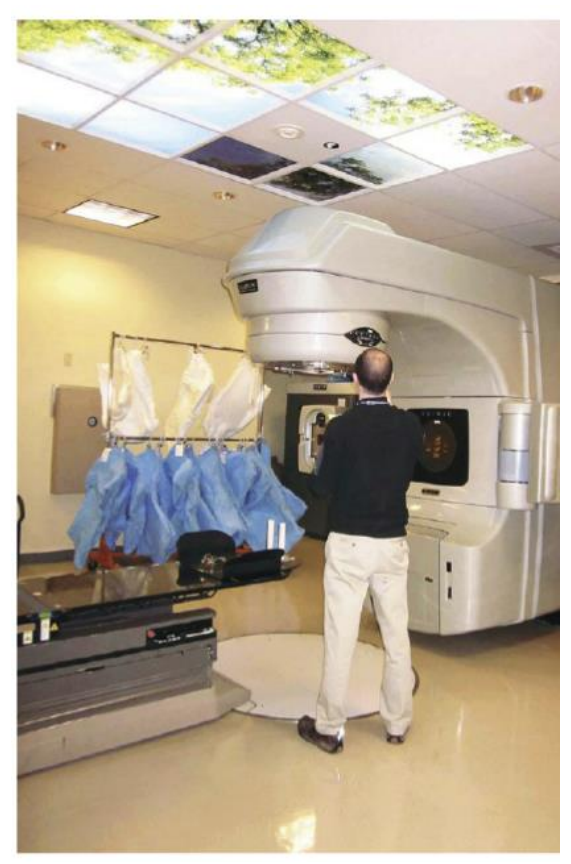

Figure 2.2.Photograph of a LINAC used in external radiation therapy [77]. 
The success of radiation therapy is related to the accurate delivery of dose to the tumor site while minimizing the dose delivered to healthy tissue. Therefore, the treatment is designed to deliver small doses of radiation once or twice a week for several weeks [78]. In the case of standard dose fractionation for tumors located in areas such as breast, head, and neck, oncologists may use absorbed doses ranging from 1.8 to 2.0 Gy per day, five days a week, until a dose of 60 Gy [78]. Radiation therapy is also used for palliative purposes, herein, low radiation doses ranging 2.5 to 8.0 Gy are fractioned until a dose of 20-30 Gy is reached [79]. Based on the type and stage of cancer, variations from the standard prescript dose (1.8-2.0 per day) can be used in treatments known as hyperfractionated and accelerated treatment [79]. In hyperfractionation, the total dose is delivered within the same time span, but the treatment is delivered in more fractions, as a result, the patient receives lower doses per session, distributed in two sessions per day $[79,80]$. On the other hand, in the accelerated treatment the total dose leads to larger doses per fraction in a smaller number of fractions [81]. It is important to notice that the standard dose is modified based on the patient's needs, therefore researchers continue to develop new treatment fractionation schemes based on the cancer stage, aiming to improve the quality of life of the patients.

Besides photon therapy, beams composed of different particles such as fast neutrons, electrons, and protons are currently being investigated for therapeutic purposes [82-84]. Neutrons have been studied due to their high linear energy transfer (LET) [85]. When a tumor is damaged by photons with low LET, the cells have a probability of repair, this scenario is not likely to occur when high LET particles such as neutrons are used [85]. The effectiveness of neutrons in therapy is reflected in the number of sessions since neutron therapy can be delivered in fewer sessions when compared to the number of treatments needed for photons [86]. However, neutron beam utilization in the clinic is still limited due to shielding requirements and irreversible damage to healthy tissue [87]. 
Charged particles such as protons and electrons have been studied as an alternative to photon therapy. Particularly, protons produced a highly localized energy deposition at a fixed depth, this phenomenon is associated with their characteristic Bragg peak [57,88]. Electrons are used in the clinic in combination with photons, and they have been successfully used for tumors and cancer close the skin surface [83].

\subsubsection{Internal Radiotherapy.}

Internal radiation therapy or brachytherapy is a form of radiotherapy where a radiation source is placed within the human body in close proximity or inside the affected area [89]. This type of therapy has been successfully used to treat cancer cells located in cervix, prostate, breast, and skin and it can be used alone and in combination with surgery and chemotherapy [56]. This technique is classified regarding the placement of the radioactive source inside the patient as follows: Interstitial brachytherapy is a type of treatment where sources are placed within the affected tissue [56], Treatment of tumors located in the head and neck, and prostate are examples of interstitial radiotherapy. Similarly, in intracavitary brachytherapy, the radioactive sources are placed in a body cavity near the affected area and it is commonly used to treat gynecological diseases [56]. Other types of brachytherapy include intraluminal, plaque, and intravascular when the sources are placed inside the lumen, tissue surface or within the blood vessels respectively. Another way to classify brachytherapy is according to the dose rate. Low-dose rate (LDR) brachytherapy uses dose rates ranging from 0.4 and $2 \mathrm{~Gy} / \mathrm{h}$, medium dose rate (MDR) brachytherapy delivers dose rates between 2 and $12 \mathrm{~Gy} / \mathrm{h}$, and high dose rate (HDR) brachytherapy uses therapeutic dose rates above $12 \mathrm{~Gy} / \mathrm{h}$ [56]. The choice of the radiation source is strongly related to the type and energy of radiation emitted by the radioisotope [89]. The size and shape of the sources used in brachytherapy are encapsulated by a material that will prevent radioisotope leakage and translocation $[89,90]$. 
Furthermore, the encapsulating material absorbs non-penetrating radiation emitted from the source such as low-energy gammas, betas, and alphas, which could potentially increase the dose delivered to the tissue. Some radioactive sources used in brachytherapy include Cs-137, Au-198, Ir-192 and I-125 [91]. A schematic representation of brachytherapy is shown in Figure 2.3.

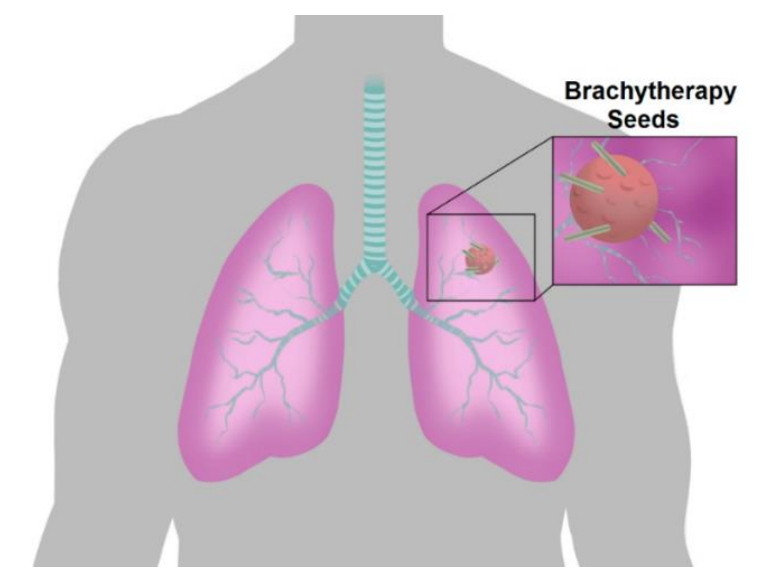

Figure 2.3. Schematic Representation of Brachytherapy. adapted from [92].

\subsubsection{Challenges of Radiotherapy.}

Radiotherapy is a technique that has been widely studied since 1896 , the first documented case of the use of radiation for curative purposes was published by Emil Grubbe to treat a patient with breast cancer [93]. Since this first case, progress in the fields of imaging and radiation therapy has favored the life expectancy of the patients [93]. Nonetheless, increasing the rate of radiation therapy as a treatment for cancer has shown side effects that include damage to normal tissues, systemic side effects such as fatigue and resistance of cells to radiation. In order to reduce side effects, physicians often prescribe smaller radiation doses and target small tumor volumes, but adverse reactions still occur [94]. Radiation therapy adverse reactions are divided into stochastic and deterministic effects. Within the stochastic, there is no particular dose threshold at which they occur, but larger doses are thought to increase the probability of occurrence, but not the severity: for example, secondary malignancies. Deterministic effects, on the other hand, have a threshold, 
and higher doses worsen the effects. Some of the side effects presented in patients treated with radiotherapy include: neurological and cardiac disorders, nausea, diarrhea, headache, and fever [95]. Since severe side effects are still observed in patients, using new radiation therapy technologies such as IMRT and image-guided radiation therapy have shown to reduced treatment adverse reactions. In spite of the use of new radiation therapy aids for treatment, side effects are still a problem with many patients [96]. Furthermore, the resistance of the tumor to the effects of radiation lead physicians to increase the radiation dose in order to destroy malignant cells [96]. As the patient's life expectancy increases, the interest in new targeted radiotherapy strategies also increases. Briefly, targeted therapy includes a set of techniques aiming to reduce tumor growth and progression by using chemical compounds in conjunction with traditional cancer treatments such as radiotherapy or chemotherapy. The overall goal of targeted therapy is to increase its efficacy while minimizing side effects.

\subsection{Radiosensitization.}

Although radiation therapy has been studied for more than a hundred years now, there are still challenges and medical complications, that make difficult the use of radiotherapy alone to decrease tumor size, while allowing for a good quality of life in patients with cancer $[4,5,13,15,93]$. One way to improve radiation therapy effectiveness is through radiosensitization, a targeted therapy technique that uses chemical agents to increase the sensitivity of tumors to the effects of radiation. Radiosensitizers can be classified into small molecules, macromolecules, and nanomaterials [5].

\subsection{Types of radiosensitizers.}

\subsubsection{Small molecules.}


Small molecules such as oxygen and oxygen-based molecules were one of the first available radiosensitization alternatives, these molecules are known to fix and amplify the damage produced by ionizing radiation within the human body $[97,98]$. Radiosensitizers in this category benefit from the indirect cell damage that occurs in conventional radiation therapy which is based on the generation of free radicals, followed by cell death. In conventional radiation treatments, the damage caused by radiation is easily repaired by molecules such as glutathione, a molecule known to neutralize free radicals inside cells, hence reducing radiotherapy effectiveness [5]. The presence of oxygen will enhance the damage by increasing the number of ROS produced during treatment, especially in low oxygen areas (hypoxia) found in most tumors [99].

One of the first methods to increase the amount of oxygen level in tumor cells is through hyperbaric oxygen, a methodology that involves pure oxygen intake in a specially designed chamber. This treatment is also used to improve oxygen supply to damaged tissue for posterior healing [100]. Another method to increase oxygen levels in tumors is the injection of hydrogen peroxide to the tumor [5]. Moreover, increasing the oxygen supply using hemoglobin, a substance naturally present in the human body known to be the primary carrier of oxygen prior to or during radiotherapy, has shown some promising results regarding the reduction of hypoxia on tumors [101]. Nowadays, small molecules radiosensitization is focused on chemical compounds containing a nitro-groups such as nitrobenzene and nitro-imidazole, molecules with the capability to enhance cell damage caused by free radicals in a similar manner to oxygen. One of the most successful molecules is 2-nitro imidazole, which showed beneficial radiosensitization effects in almost all solid tumors [102,103].

Small-molecules were the first radiosensitizers studied, hence they have well-established results showed in preclinical and clinical trials [5]. Research on small molecule radiosensitization is based 
on the design and analysis of pharmacokinetics and pharmacodynamics of the different molecules, by using computer simulation [5]. Furthermore, the discovery of the radiosensitization response in macromolecules and nanomaterials has enabled new fields of study related to the conjugation of small molecules with other types of sensitizers.

\subsubsection{Macromolecules.}

In addition to small molecules, macromolecules such as miRNAs, proteins, peptides, and oligonucleotides have been studied as radiosensitizers [5]. Macromolecules are long-chain molecules that bind to the DNA to increase the radiosensitivity of the cells, these molecules have been used for a long time as antibodies in conjunction with radiation therapy. Proteins, antibodies and short peptides, present affinity to tumor cells, and upon interaction with radiation, they inhibit cell repair, inducing apoptosis [5]. Furthermore, proteins can be used as drug carriers of radionuclides for brachytherapy and targeted therapy applications. When combined with other types of radiosensitizers such as nanomaterials, targeted proteins can play a great role by focalizing and enhancing the radiation delivered to the tumor site. For example, the antibody known as antiRhoJ was used to orient $\mathrm{Au}$ nanoparticles to tumor vessels, generating promising research on targeted radiosensitizers [104]. Radiosensitizers such as oligonucleotides interfere with gene reproduction by binding to cells and degrading their replication processes [105-107]. Other macromolecules which evidence radiosensitization are the following: antibodies SYM004 and AllB2, proteins such as miRNAs, DZ1 and NKTR-214, among others [5]. Overall, when compared with small-molecules sensitizers, macromolecules have shown better targeting effects, and they are easy to design and synthesize [5]. The development of technologies for macromolecules analysis such as pharmacokinetics and stability studies within the human body could help to the better understating the binding and radiosensitization processes on macromolecules on tumors. 


\subsubsection{Nanomaterials.}

Nanomaterials are inorganic compounds able to scatter, absorb, and emit high energy particles such as photons, electrons, and fluorescent $\mathrm{x}$-rays $[7,13,39]$. This could potentially allow for a better targeting strategy by localizing the damage to a small volume of tissue, the use of nanomaterial radiosensitizers is being referred to as Nanoparticle Enhanced X-ray Therapy or NEXT [4]. Particularly, nanoparticles composed of high atomic number elements can absorb the X-rays photons leading to the different photo-emissions that will ionize the medium creating ROS that will interact with both the nanoparticles and the media, causing enhanced cellular damage and increasing cell death $[3,5]$

Nanomaterials have a mean particle size below the kidney filtration threshold, hence nanoparticles can be secreted through the urinary system, showing low accumulation toxicity. Furthermore, within the human body nanoparticles have shown favorable kinetic profiles, and low toxicity, which make biocompatible metal-oxide and metallic nanomaterials, promising candidates as radiosensitizers [108]. Moreover, nanoparticles in the absence of radiation produce physical and chemical changes on cells that include morphology modification, cell gap creation, and cell movement restrictions [5,109]. Another important application of nanoparticles for therapeutic applications is as drug carriers, herein, nanoparticles are used to delivered chemical agents to enhance tumor damage [104]. Overall, nanoparticles used as radiosensitizers or nanocarriers represent a promising route to the development of new targeted treatments. In this work, a new platform, where metallic nanoparticles are supported on metal oxides creating a nanocomposite was investigated evidencing the effectiveness as radiosensitizers. In this case, we used the combined effect of ROS produced by $\mathrm{TiO}_{2}$ and the photo-emissions released by Au upon X-ray 
irradiation. Below, a literature review of $\mathrm{Au}$ and $\mathrm{TiO}_{2}$ used separately as a radiosensitizer is presented.

\subsubsection{Radiosensitization of Gold}

The pioneer experiments with Au as radiosensitizers were conducted by Hainfeld et al. in 2004. In this work, $\sim 2 \mathrm{~nm}$ nanoparticles at an absorbed dose of $30 \mathrm{~Gy}$ (using $250 \mathrm{kVp} \mathrm{X}$-rays) were injected in mice mammary carcinomas. Results have shown an increase in the one-year survival of mice from $20 \%$ to $86 \%$ using Au nanoparticles at a concentration of $2.7 \mathrm{~g}$ of Au per mice $\mathrm{kg}$ [110]. Since Hainfeld et al, several studies have demonstrated the ability of Au nanoparticles to increase the radiosensitivity of cells for in-vitro and in-vivo experiments. The idea of using Au as radiosensitizer arises from the higher mass energy-absorption coefficient of Au compared to soft tissue and water. For charged particle equilibrium conditions. The mass energy-absorption coefficient is a measure of the average fraction of the photon's energy absorbed by the medium. Otherwise, the mass energy-absorption coefficient is the fraction of the photon energy that is transferred to charged particles that is not loss due to radiative losses. The mass energy-absorption coefficient is denoted by $\mu_{\mathrm{en}} / \rho$ and usually, it has units of $\mathrm{cm}^{2} / \mathrm{g}$ or $\mathrm{m}^{2} / \mathrm{kg}$ [111]

Therefore, Au radiosensitization has shown to vary with parameters such as incoming photon energy, Au particle size, and concentration. In regards to the X-ray beam energy, different interactions mechanisms take place based on the energy of the beam. When the energy of the incoming photon is in the range of $10-500 \mathrm{KeV}$, the photoelectric effect is the dominant effect. For photons between $0.5 \mathrm{MeV}$ and $1.02 \mathrm{MeV}$ Compton scattering is observed, where the production of Compton electrons will lead to subsequent photoelectric effect. Finally, pair production occurs for photon energies higher than $1.02 \mathrm{MeV}$ where additional positrons will be produced [13]. 
The particle size plays an important role in the radiosensitization effect. To date, experiments have been carried out using Au nanoparticles with a mean particle size ranging from $\sim 2 \mathrm{~nm}$ to $\sim 50 \mathrm{~nm}$ [3]. Results showed that smaller Au nanoparticles produce higher levels of ROS, confirming the chemical enhancement role of Au nanoparticles surface. The enhanced chemical activity of Au nanoparticles is due to $\mathrm{O}_{2}^{-\bullet}$ and $\mathrm{OH}^{\bullet}$ produced by the interaction of X-rays with the surface atoms of the nanoparticles [3]. Furthermore, in-vitro toxicity experiments evidenced that small nanoparticles had higher toxicity effect in cells than larger nanoparticles. In regards to dose enhancement, researchers have found that smaller particles emit a larger fraction of Auger and delta electrons than bigger particles. The reason behind this phenomena is that auger and delta electrons are absorbed within larger nanoparticles [18]. Monte Carlo simulations of radiosensitization effects in Au nanoparticles showed that 2.6 Auger and delta electrons escape per photoelectric event from Au nanoparticles with a particle size of $1.9 \mathrm{~nm}$, while less than 1 Auger and delta electrons escaped from Au nanoparticles of $100 \mathrm{~nm}$ [18]. It is important to notice that there are discrepancies regarding the optimal size of Au nanoparticles for radiosensitization purposes. Some authors claim that nanoparticles of $\sim 50 \mathrm{~nm}$ in diameter, represent the most efficient radiosensitizer with an enhancement factor of 6 [13]. In contrast, other studies affirm that when the diameter of the Au nanoparticles is increased from 8 to $92 \mathrm{~nm}$, the enhancement factor raised up a value of to 3 [15]. The inconsistency of the results is due to different parameters used in experiments between investigations such as nanomaterial concentration, type of cells, and voltage of the X-ray source. Therefore, further experimentation in the field is needed to clarify the relevance of these parameters in the dose enhancement provided by Au nanoparticles.

\subsubsection{Radiosensitization of $\mathrm{TiO}_{2}$}


Titanium dioxide has been shown to be useful for killing cancer cells in phototherapy applications. Interestingly, $\mathrm{TiO}_{2}$ nanoparticles have in their surface hydroxide groups that increase the production of free radical such as $\mathrm{OH} \bullet$ and $\mathrm{H} \bullet[112]$. The uses of $\mathrm{TiO}_{2}$ as radiosensitizer involves three processes: the excitation, bulk diffusion, and surface transfer, of induced charge carriers [22]. The excitation of a semiconductor initiates with the absorption of a photon with an energy larger than its band gap $(\sim 3.2 \mathrm{eV})$. This leads to the production of charge carriers. When excited, electrons migrate to the conduction band and holes are left behind in the valence band. Afterward, the carriers travel to the surface of the nanomaterials [22]. Diffusion of charges is affected by crystal structure and particle size of $\mathrm{TiO}_{2}$. The holes react with adsorbed $\mathrm{H}_{2} \mathrm{O}$ to produce $\mathrm{OH}^{\circ}$. Meanwhile, electrons usually react with $\mathrm{O}_{2}$ to produce $\mathrm{O}_{2}{ }^{-}$, ROS that ultimately contribute to damage of malignant tissue.

There are several studies that show the photosensitization effect of $\mathrm{TiO}_{2}$, a phenomenon only useful to treat superficial tumors [22]. On the other hand, X-rays and gamma-ray sources used in radiotherapy allows for treatment of tumors at any specific depth by tuning the energy of the incoming photons. Most of the available literature focuses on $\mathrm{TiO}_{2}$ as photosensitizer with scarce reports on their behavior as a radiosensitizer. One of the few in-vitro studies that evaluated $\mathrm{TiO}_{2}$ radiosensitization showed that $\mathrm{TiO}_{2}$ combined with an absorbed dose of $2 \mathrm{~Gy}$, produced more DNA damage than irradiation alone. They also found that elongated nanoparticles internalize into cells more effectively than spherical nanoparticles $[9,112]$.

\subsection{Nanoparticle radiation enhancement.}

Radiation enhancement by nanoparticles is based on the localized increase of absorbed dose upon irradiation. At first, this enhancement was thought to be caused by the increased absorption of Xrays and the emission of secondary radiation by the nanomaterials, leading to the generation of 
electrons and radicals to the surrounding media. This effect leads to increasing the dose deposited in matter [113]. However, it was observed that nanoparticle dose enhancement was a complex mechanism, and needed to be studied based on the type of emissions and subsequent damage caused to the surrounding media [113]. To date, there are three different mechanisms of enhancement known as physical enhancement, chemical enhancement, and biological enhancement $[6,113]$.

\subsubsection{Physical Enhancement.}

The addition of nanomaterials into a medium and their interaction with X-rays irradiation causes an overall increase in the photon absorption of the medium $[4,18]$. The absorption becomes more evident when nanomaterials are introduced to an environment composed of low atomic mass number elements such as water or tissue [13]. X-rays absorption of nanomaterials leads to an increment in the energy deposition and dose delivered to the surrounding medium, this enhancement caused by nanomaterials is known as physical enhancement [7]. Physical enhancement can lead to single or double-strand damage of DNA molecules, cell death, protein damage, and ultimately tumor destruction [5]. In physical enhancement, the damage is linearly proportional to the increased absorption of X-rays by the particles [114].

In order to properly understand physical enhancement, the concept of mass energy-absorption coefficient needs to be explained in detail. The mass energy-absorption coefficient is a measure of the average fraction of the photon energy transferred to the charged particles in the medium, minus the energy that the photon lose by radiative interactions $[111,115]$. The kinetic energy gained by the charged particles as a result of their interaction with the photons depends on different factors such as the absorber chemical composition, the dimensions of the absorber, incident photon energy, among others [114]. The energy imparted to the media causes different physical, chemical 
and biological effects associated with exposure to ionizing radiation. The mass energy-absorption coefficient $\left(\mu_{e n} / \rho\right)$ can be calculated by estimating the incident X-ray intensity, or experimentally by measurements made with an ionization chamber [115]. The mass energy-absorption coefficient is also related with the absorption cross section per atom $\sigma_{\text {en }}$, which is the probability of interaction of photons with the media, the units of the absorption cross section are barns/atom, where 1 barn $=10^{-28} \mathrm{~m}^{2}$ [115]. The equation that relates mass energy-absorption coefficient with the absorption cross section is shown in equation 1 . Where $\mathrm{N}_{\mathrm{A}}$ is the avogadro's number and $\mathrm{M}$ is the atomic or molecular weight of the media. Furthermore, the mass energy-absorption cross section can be expressed as the summation of different interaction mechanism as shown in equation 2 , where $\sigma_{\text {incoh }}, \tau, k$ and $\sigma_{p h . n}$ are the incoherent scattering cross section, the photoeffect cross section, the pair production cross section and the photonuclear cross section respectively. The $f$ represents their respective probability of interaction [115].

$$
\begin{gathered}
\frac{\mu_{e n}}{\rho}=\sigma_{e n}\left(\frac{N_{A}}{M}\right)(1) \\
\sigma_{\text {en }}=\sigma_{\text {incoh }} f_{\text {incoh }}+\tau f_{\tau}+k f_{k}+\sigma_{p h . n} f_{p h . n}(2)
\end{gathered}
$$

Physical enhancement is caused by the interaction of X-rays with the electrons in atoms within the nanomaterials, and it is related to the cross-section for each interaction [114]. The interaction mechanisms of X-rays and electrons depends on parameters such as the atomic number and energy of the photons. Physical enhancement benefits from atoms with a high atomic mass number. For instance, a material with high atomic number elements has a higher X-ray absorption cross-section than low atomic number compounds [6]. For example, the photoelectric cross section of an atom $\left(\sigma_{p e}\right)$ is proportional to $\sim(\mathrm{Z} / \mathrm{E})^{3}$, where $\mathrm{E}$ is the energy of the incident photon and $\mathrm{Z}$ is the atomic number of the material being excited [4]. Physical enhancement is 
related to the increase in absorbed dose produced by nanoparticles from physical processes such as photoelectric effect, Compton scattering and pair production. Particularly, a material that has consistently exhibit physical enhancement is Au, an element with high mass-energy absorption coefficient. In Figure 2.4a, a comparison between the mass energy absorption coefficient of water and $\mathrm{Au}$ is presented and in Figure 2.4b, the ratio between the mass energy-absorption coefficient of $\mathrm{Au}$ and water is presented, showing the superior photon absorption of Au with respect to water [17].
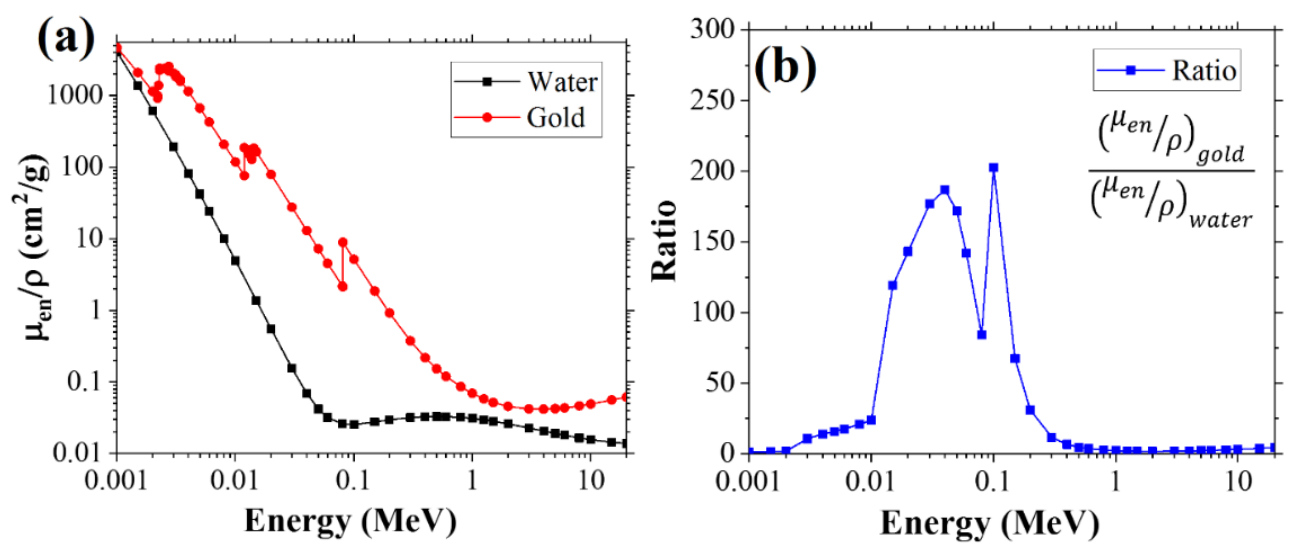

Figure 2.4. a) The mass-energy absorption coefficient for water and Au. b) The ratio between mass energy-absorption coefficients of Au over water is shown as a function of energy [17]. The process that follows absorption is emission, herein, electrons are emitted from the nanoparticles as a result of X-ray photons being absorbed by the atoms within the particle [18]. For materials composed of high atomic number, and $\mathrm{X}$-ray energies in the $\mathrm{keV}$ range, the highest probability event is the emission of photoelectrons. Since the process of photoelectric effect involves a hole created in the atomic orbitals, other processes such as the Auger electron emission occur $[13,18]$. Auger electrons production takes place when a photon is emitted after the hole is filled, this photon gets absorbed by the atom and a bound electron is ejected. In this process, two electrons are emitted: one photoelectron and one Auger electron. For Au atoms, a K hole on 
average produces nearly five electrons [114]. The first electron released from the atom is the primary photoelectron, and the following emissions are Auger electrons, Figure 2.5 shows the different processes that can take places within an Au atom when physical enhancement occurs.

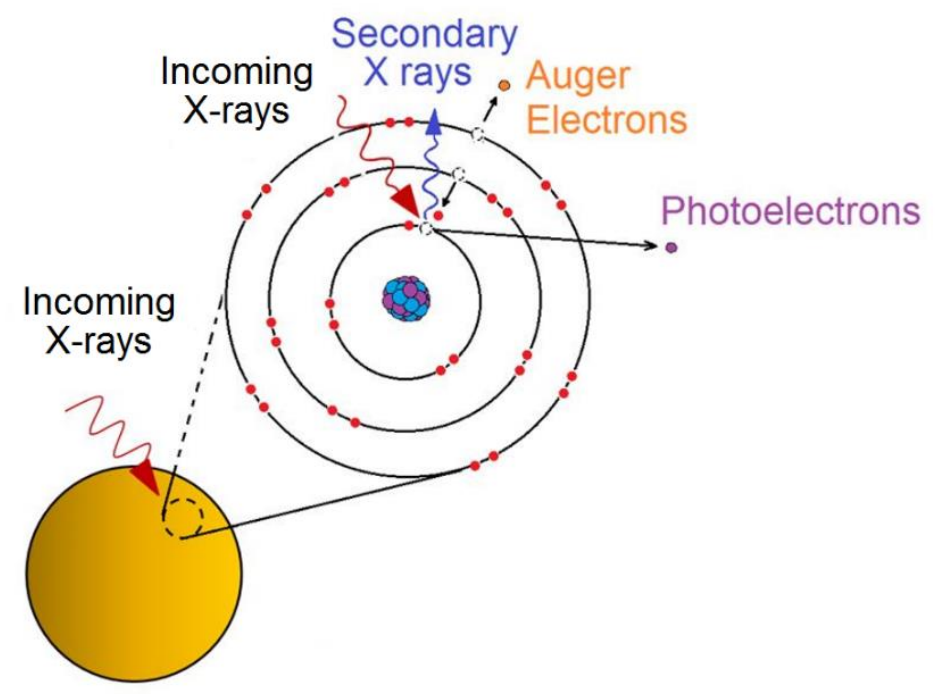

Figure 2.5. Schematic representation of physical enhancement on High Z nanoparticles.

\subsubsection{Chemical Enhancement.}

Chemical enhancement is related to the catalytic properties of the nanomaterial embedded in specific media. Chemical enhancement is measured through indirect methods such as fluorescence by quantifying a fluorescent signal in the presence and absence of nanomaterials [5]. The detected signals are the result of ROS interaction with the fluorescent probe. On in vitro experiments, when DNA strand breaks, the detected ROS signal is associated with the fluorescence emitted from dyestained cells [5]. Chemical enhancement is characterized by an increased ROS production due to the interaction of nanomaterials with the products from radiolysis of water [116]. The reduction of ROS when nanoparticles are present has been observed in a process known as anti-enhancement [117]. 
One of the challenges in radiosensitization is to isolate physical enhancement from chemical enhancement. Chemical enhancement, and hence ROS production, is dependent on X-ray dose rate [118]. In contrast, physical enhancement does not depend on dose rate but rather on the energy X-ray beam [117]. Catalytic reactions are critical for radiosensitization processes and especially chemical enhancement, where the production of ROS is triggered by the nanomaterial interaction with X-rays and ROS created by radiolysis of water [5]. Nanoparticles have shown to be effective catalysts, because they have large surface areas per unit volume, making a higher number of catalytic sites available when compared with bulk materials [119]. Furthermore, the atoms in the nanomaterial surface are more reactive, consequently, these surface atoms can potentially interact with the medium in a process that lowers reaction energy barriers and facilitates catalytic processes [116]. ROS production and chemical enhancement are depicted in Figure 2.6.

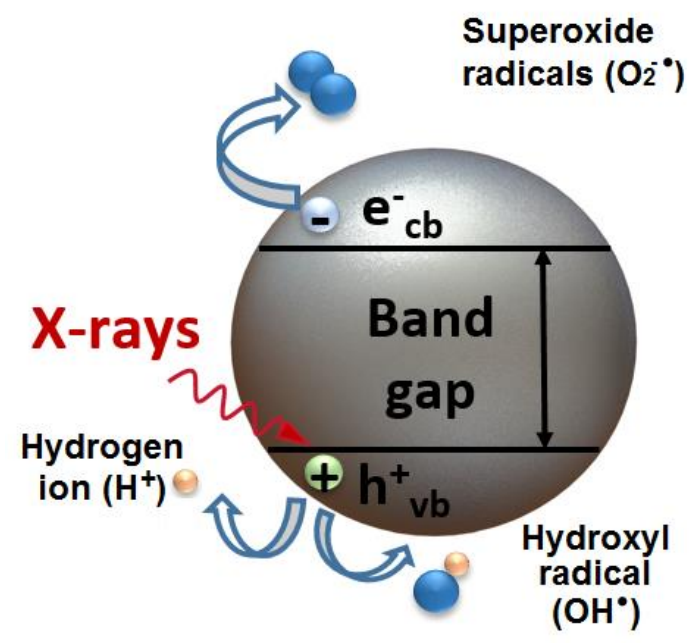

Figure 2.6. Chemical Enhancement Produced by metal oxides nanoparticles.

The size of nanoparticles is one of the most important parameters in radiosensitization since it controls the number of atoms available in the surface, a feature that will determine the catalytic properties of the nanomaterial. Furthermore, the particle size determines the surface area per unit 
mass [119]. The shape of nanoparticles also influences the catalytic activity, since nanomaterials with different shapes can support different reaction pathways [116]. For example, needle-shaped nanostructures exhibit complex local catalysis pathways at their narrowest point [112]. Besides size and shape, the catalytic activity is influenced by surfactants and ligands used to synthesize nanomaterials. Surfactants can affect the particle surface charge density, improve the solubility of nanoparticles in media, increase cellular uptake of nanomaterials, and enable functionalization processes that could guide particles to a specific tumor site [5]. Surfactants and ligands could increase chemical enhancement because they can scavenge or produce more ROS [120]. As a result, the chemicals and synthesis route to fabricate the nanomaterial may influence the catalytic properties.

The interaction of X-rays with nanomaterials embedded in an aqueous media triggers ROS production. The main species encountered in the radiolysis of water are: $\mathrm{OH}^{\bullet}, \mathrm{O}_{2}^{*}, \mathrm{e}_{\mathrm{aq}}^{-}, \mathrm{H}_{2} \mathrm{O}_{2}$, hydrogen atoms $\left(\mathrm{H}^{+}\right)$, and singlet oxygen $\left(\mathrm{O}_{2}\right)$ [116]. ROS production varies as a function of time for pulse radiolysis, on the other hand, ROS production does not vary as a function of time for continuous radiation [116]. Table 2.1. Reactive species yield in pulse radiolysis and steady-state radiation [116]. The table presents the yield of these species with their $G$ values, which are the number of species generated per $100 \mathrm{eV}$.

Table 2.1. Reactive species yield in pulse radiolysis and steady-state radiation [116].

\begin{tabular}{|c|c|c|c|c|c|}
\hline & $\mathbf{e}^{-}{ }_{\text {aq }}$ & $\mathbf{H \bullet}$ & $\mathbf{H}_{2}$ & $\mathbf{O H} \bullet$ & $\mathbf{H}_{2} \mathbf{O}_{2}$ \\
\hline Pulsed (1 ps) & 4.8 & 0.62 & 0.15 & 5.7 & \\
\hline Steady State & 2.7 & 0.55 & 0.45 & 2.8 & 0.7 \\
\hline
\end{tabular}


Absorption of X-rays by nanomaterials is dependent on X-ray energy. For example, in a medium such as water, $\mathrm{X}$-rays with lower energies than $20 \mathrm{keV}$ and higher than $100 \mathrm{keV}$ are absorbed more strongly by Au than by water [111]. This information can be derived from the ratio between the mass absorption coefficient of $\mathrm{Au}$ to respect to water presented in Figure 2.4a. Chemical enhancement is driven by reactive oxygen species generated in water through Compton scattering of X-rays and through direct absorption of X-ray by nanomaterials. X-rays with an energy spectrum from 10 to $150 \mathrm{keV}$ generate both chemical and physical enhancements. Nonetheless, photon energies from 30-100 keV have shown to favor physical enhancement and low energy photons in the range of $10-15 \mathrm{keV}$ and high energy photons $100-150 \mathrm{keV}$ favor chemical enhancement [116].

\subsubsection{Biological Enhancement.}

Biological enhancement is associated with the damage that occurs to the DNA in the cells due to the combined action of radiation and nanoparticle presence [5]. It has been found that biological enhancement may or may not require increased absorption of X-rays by nanomaterials. Nanomaterials bind to cells in order to cause enhanced damage under X-ray irradiation, and the damage can be further amplified [19]. Cells components such as mitochondria and nuclear DNA can be damaged by electrons emitted from nanomaterials or by the action of ROS that nanomaterials generate when irradiated with X-rays [121]. One hypothesis is that the mechanisms of physical and chemical enhancement are combined to amplify cell damage. Hainfeld et al. were the first to report an animal study of Au nanoparticle radiosensitization. They observed a $86 \%$ increase in survival rate compared to $20 \%$ with $\mathrm{X}$-rays alone when using $\mathrm{Au}$ nanoparticles with a particle size of $1.9 \mathrm{~nm}[121]$. 
Biological enhancement can also be produced by the presence of the nanomaterial in the absence of irradiation, in a process known as indirect enhancement $[5,19]$. This type of enhancement is observed when nanomaterials block DNA repair pathways by binding to proteins, diminishing DNA repair functions [5]. A schematic representation of biological enhancement is depicted in Figure 2.7.

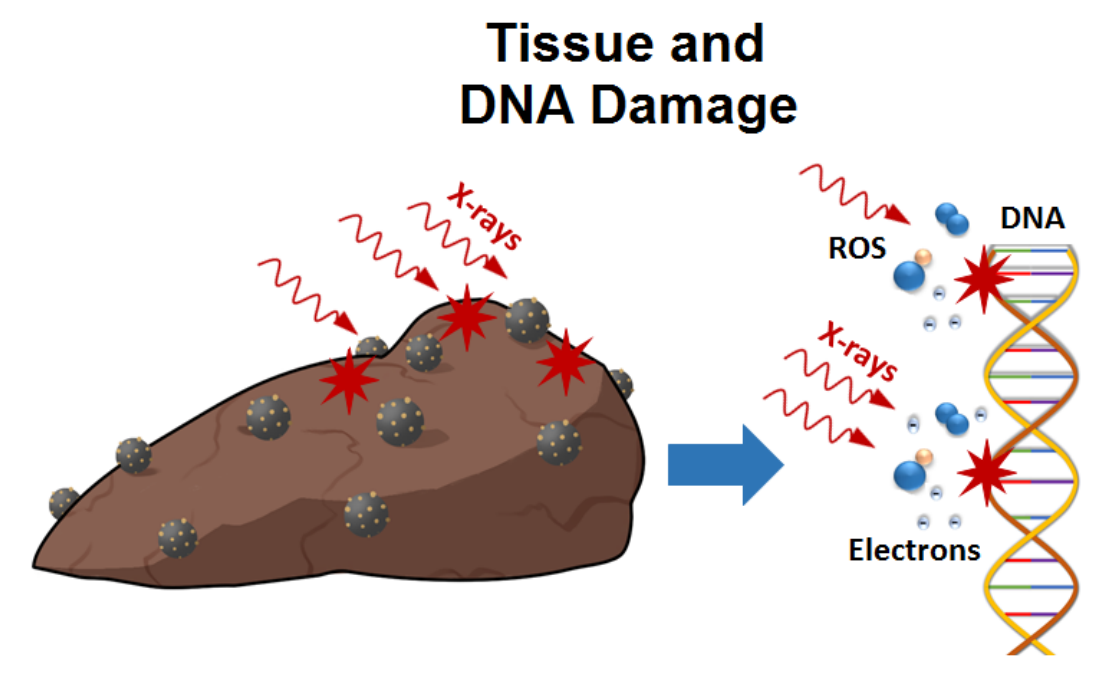

Figure 2.7. Biological enhancement of nanoparticles. 


\section{Chapter 3: Overview of fabrication methods to synthesize gold supported titania}

The study of Au nanoparticles synthesis is of valuable interest since the catalytic properties of $\mathrm{Au}$ nanoparticles can be utilized in fields such as targeted therapy, imaging, and catalysis [14,50]. It is well known that the catalytic activity of Au nanoparticles is directly related to their particle size [122]. Thus, research on $\mathrm{Au}$ nanoparticle fabrication methods is directed towards techniques that offer nanoparticles with small particle size, narrow particle size distribution, and an adequate stabilization that prevents nanoparticle aggregation [21,39]. The use of solid supports has shown to be a successful method that helps to control Au nanoparticle growth and prevents aggregation [21,39]. This is particularly important for Au since their catalytic activity diminishes as the particle size grows beyond $10 \mathrm{~nm}$ [122]. Specific characteristics of the support such as surface area, particle size, the presence of surface hydroxyl groups, the density of defects, and crystal phase could increase the number of catalytic active sites and influence the deposition of Au nanoparticles onto its surface $[119,122]$. Furthermore, a wise combination of nanoparticles with supports can provide synergistic properties not presented by the substrate nor the nanoparticles alone.

The most commonly used methods to support Au nanoparticles onto metal oxides supports are adsorption and deposition-precipitation [29,122,123]. These methods rely on chemical reducing agents to reduce metallic ions into atoms, followed by a controlled process where particles coalesce and grow. Other methods that have been explored when supporting $\mathrm{Au}$ nanoparticles on $\mathrm{TiO}_{2}$ include: sonochemical synthesis, the sol-gel method, deposition-precipitation, photochemical and radiolytic synthesis, below an explanation of each method is given: 


\subsubsection{Sonochemical method.}

Sonochemical synthesis of nanoparticles is carried out in an ultrasound bath. Herein, a metallic salt, support, and organic species are added to a container and sonicated all together. Organic species such as oleic acid and polyvinylpyrrolidone (PVP) break up into smaller fragments during sonolysis process, activating metal precursors reduction processes [124]. This also enables the deposition of metal nanoparticle on the support material [123]. Sonochemical synthesis is cleaner than conventional chemical methods, and it requires few post-processing cleaning treatments. $\mathrm{Au}$ nanoparticles with a mean particle size of $14.3 \mathrm{~nm}$ have been successfully deposited on $\mathrm{TiO}_{2}$, in the presence of polyethylene glycol (PEG)[125]. Furthermore, nanoparticles made of $\mathrm{Pt}(2.1 \mathrm{~nm})$ and $\mathrm{Pd}(3.6 \mathrm{~nm})$ have been synthesized by this method onto $\mathrm{TiO}_{2}$ [125]. Sonochemical methods offer low by-product deposition on the synthesized particles, nonetheless, there are some disadvantages associated with this technique such as lack of control on the particle size distribution and loading, this technique also present issues associated to industrial scalability $[124,126]$.

\subsubsection{Sol-Gel Method.}

The sol-gel method is a synthesis method that allows for a strong chemical interaction between $\mathrm{Au}$ and $\mathrm{TiO}_{2}$ by creating oxygen and hydroxyl bridges formed between Au colloids and the titania support during the process of hydrolysis [123]. This Au nanoparticle synthesis method begins with a mixture of a metallic salt, the support precursor, a reducing agent, and a polymeric agent in an aqueous solution. Then, the solution is heated, hydrolysis of water takes place and Au complexes are formed. The solution is collected, and a post-synthesis calcination at $600{ }^{\circ} \mathrm{C}$ for $6 \mathrm{~h}$ allows for nanoparticle formation [127]. The material precursors used in this method are traditionally metal alkoxides and chlorides, these compounds can be easily decomposed in water allowing for a fast hydrolysis process [128]. The calcination process favors the formation of the anatase of $\mathrm{TiO}_{2}$ and 
a reduction of the $\mathrm{TiO}_{2}$ surface area is observed. Presence of copolymers as gelatin agents enhances the surface area of the synthesized $\mathrm{TiO}_{2}$ up to twice that of pure $\mathrm{TiO}_{2}$ [129]. Moreover, reports using the sol-gel method have shown an increase in the particle size when increasing nanoparticle loading, and growth of smaller golf particles observed on $\mathrm{TiO}_{2}$ anatase with high surface area [123]. A schematic representation of the overall process is depicted in Figure 3.1.

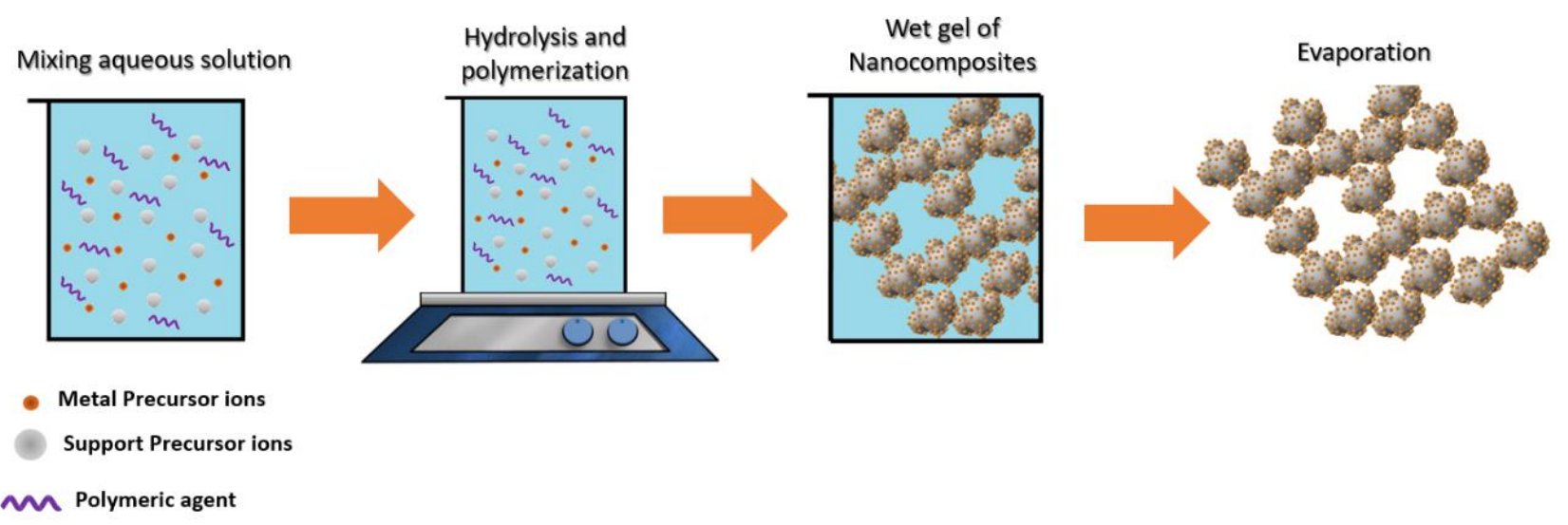

Figure 3.1. Schematic representation of Au supported nanoparticles by the sol-gel method.

\subsubsection{Deposition-precipitation Method.}

Deposition-precipitation as a method for the synthesis of Au-supported titania nanoparticles, is known to produce "reference catalysts" by the world gold council, due to its high degree of reproducibility and good quality for catalytic applications [46]. This method is based on the deposition of hydrated oxides and hydroxides onto the support in a procedure that allows for a gradual $\mathrm{pH}$ increase in the solution, heated to relatively low temperatures, in which the metallic precursor and solid support are suspended [46]. The deposition of $\mathrm{Au}$ ions onto the $\mathrm{TiO}_{2}$ surface starts with a heating process $\sim 80{ }^{\circ} \mathrm{C}$ in the presence of ligands such as $\mathrm{NaOH}$, urea or sodium carbonate $\left(\mathrm{Na}_{2} \mathrm{CO}_{3}\right)$, that will slowly raise the $\mathrm{pH}$ of the solution [24]. The subsequent precipitation of Au nanoparticles onto the $\mathrm{TiO}_{2}$ surface is achieved through calcination at high temperatures for several hours (i.e $300{ }^{\circ} \mathrm{C}$ for 3 hours), then the resultant powder is collected and washed in order 
to remove undesired species [123]. This method has been widely studied because it leads to the production of small Au nanoparticles with an average particle size of $\sim 5 \mathrm{~nm}$, a particle size suitable for a variety of catalytic reactions [123]. The particle size and shape of the as-synthesized Au nanoparticles strongly depends on the parameters used deposition of $\mathrm{Au}$ nanoparticles on the $\mathrm{TiO}_{2}$ surface, high catalytic activity has been linked with a $\mathrm{pH}$ of the reaction between $7-8$, since the electrostatic attraction between $\mathrm{Au}$ and $\mathrm{TiO}_{2}$ is higher at this $\mathrm{pH}$ level [46]. Synthesis of $\mathrm{Au} @ \mathrm{TiO}_{2}$ with the deposition precipitation technique has been made tailoring the $\mathrm{pH}$ from 4.5, 6 to 9, and calcination temperature from 200 to $400{ }^{\circ} \mathrm{C}$ [123]. Results regarding particle size showed that higher $\mathrm{pH}$ and temperature causes agglomeration due to the sintering processes occurring at the $\mathrm{Au}$ and $\mathrm{TiO}_{2}$ interface [123], whereas acidic $\mathrm{pH}$ causes the gold-chlorides species to dilute during the heating process, preventing the formation of hydroxides. The smallest Au particle size was formed by calcining the composite at $200{ }^{\circ} \mathrm{C}$ at a $\mathrm{pH}$ of 9 [123]. A schematic representation of the deposition-precipitation method is showed in Figure 3.2.

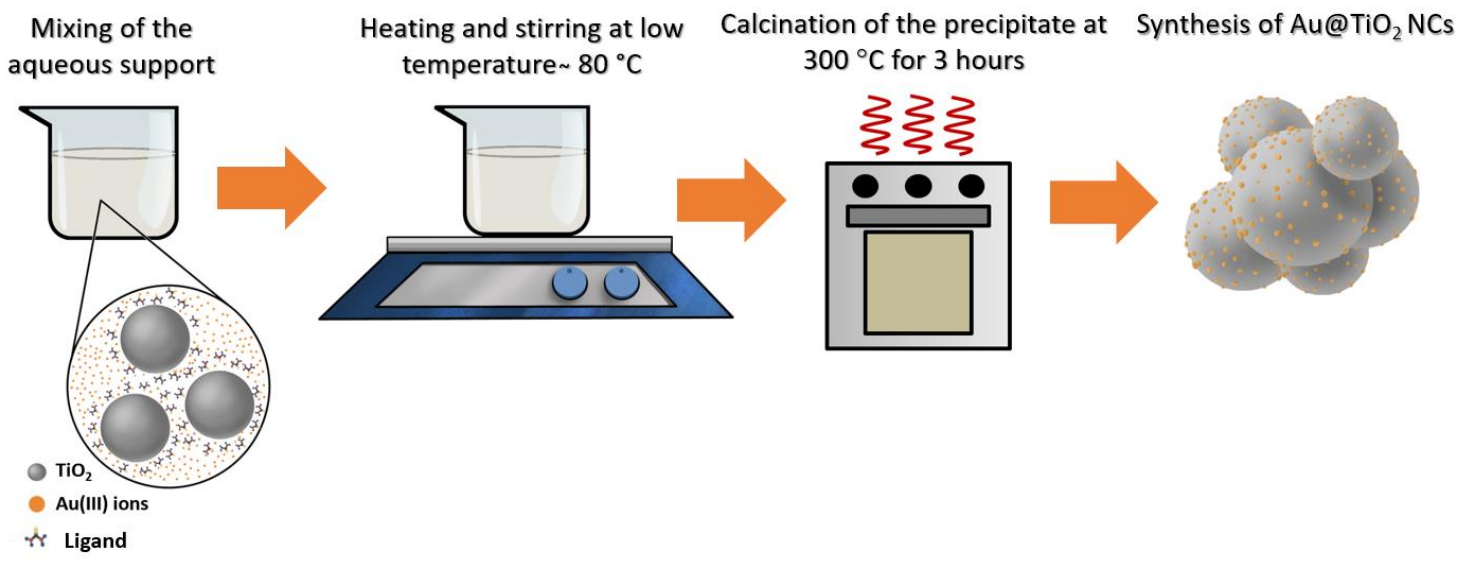

Figure 3.2. Schematic representation of the synthesis of Au supported titania by the Depositionprecipitation method. 


\subsubsection{Photochemical deposition method.}

One of the alternatives to traditional chemical synthesis is the photochemical deposition method of $\mathrm{Au}$ onto $\mathrm{TiO}_{2}$. The photochemical reduction of $\mathrm{Au}$ is carried out under UV-light at energies below $60 \mathrm{eV}$ [130]. Through this method, nanoparticles with high purity can be fabricated, due to the absence of chemical reducing agents [123]. The interaction of the semiconductor support with light produces electron-hole pairs, these electrons will cause the reduction of Au precursors to $\mathrm{Au}$ metal, the atoms will be subsequently adsorbed onto the surface of the semiconductor and particles will nucleate and grow [123]. On the other hand, holes will interact with the metallic precursor bringing it to higher oxidation levels, thus scavengers such as alcohols need to be present to avoid accumulation of positive charge during the photo-deposition process [123]. Synthesis of $\mathrm{Au} @ \mathrm{TiO}_{2}$ nanocomposites using photochemical reduction has been carried out using a metal precursor $\left(\mathrm{HAuCl}_{4}\right)$, in a medium of water/alcohol [131]. Upon light interaction with the medium, photolysis of water occurs and water molecules break down into species such as $\mathrm{e}_{\mathrm{aq}}^{-}, \mathrm{H}^{+}$and $\mathrm{OH}^{*}$. Alcohols such as isopropanol or methanol are added to the mixture so they interact with oxidizing species in solution producing a higher number of reducing species such as $\mathrm{e}_{\text {aq }}^{-}$and $\mathrm{H}^{+}$, that reduce metal ions to lower oxidation states [123]. Au nanoparticles have been supported on $\mathrm{TiO}_{2}$ through the photochemical method using UV lamps with power ranging from $3 \mathrm{~W}$ to $300 \mathrm{~W}$, using reaction times from 0.5 to 3 hours [123]. The obtained particle size varies from $3 \mathrm{~nm}$ to $20 \mathrm{~nm}$ and it has been found that the size strongly depends on synthesis parameters such as reaction time, the chemistry of the media and power of the lamp [123]. 


\subsection{Radiolytic synthesis of nanomaterials.}

Most of the techniques to synthesize supported Au nanoparticles are based on the reduction of a metallic precursor in the presence of reducing and stabilizing agents. Among the irradiation-based techniques, the most widely studied method is UV-irradiation [36]. Nonetheless, ionizing radiation using high energy photon beams such as $\gamma$-rays and X-rays have shown to be an outstanding method for nanomaterials production. An important advantage of radiolytic synthesis is that one of the main reducing agents is $\mathrm{e}^{-}$aq, species allow for uniform ion reduction in solution, leading to the formation of homogeneously distributed $\mathrm{Au}$ seeds. Thus, homogeneously dispersed nanoparticles with narrow particle size distributions are obtained [35,52]. Due to the advantages of this method, radiolytic synthesis using gamma rays has been recently used to produce nanocomposites on carbonaceous, polymeric and oxides supports. Some nanocomposites synthesized to date include Ag@SWCNTS, Ir@graphene oxide, Pd@Polyaniline, Ni@TiO2 and $\mathrm{Pd} @ \mathrm{Al}_{2} \mathrm{O}_{3}$ [35]. Nonetheless, radiolytic synthesis of nanomaterials with X-rays represents a feasible alternative to gamma rays [41]. The limited available literature about X-rays radiolytic synthesis refers to Gold-Platinum, copper, silver and Au nanoparticles deposited onto different polymeric supports such as polymethyl methacrylate (PMMA)[132], Polymeric acidpolyethyleneimine (PAA-PEI) films [132], polytetrafluoroethylene (PTFE) and polyimide (PI) films respectively $[133,134]$.

\subsubsection{Radiolysis of water.}

The reaction that occurs upon interaction of ionizing radiation and water is known to as radiolysis. When water interacts with a photon carrying an energy of $\sim 13 \mathrm{eV}$ or higher, water molecules are fragmented [35]. The interaction of ionizing radiation with water leads to the creation of species 
such as $\mathrm{e}^{-}{ }_{\mathrm{aq}}, \mathrm{H}^{\bullet}, \mathrm{H}^{+}, \mathrm{OH}^{\bullet}, \mathrm{O}_{2}{ }^{-}, \mathrm{H}_{3} \mathrm{O}^{+}, \mathrm{H}_{2}$ and $\mathrm{H}_{2} \mathrm{O}_{2}$. Equation 3, shows the different species produces during radiolysis of water.

$$
\mathrm{H}_{2} \mathrm{O} \stackrel{\text { radiation }}{\longrightarrow} \mathrm{OH} \bullet+e^{-}{ }_{a q}+\mathrm{H} \bullet+\mathrm{H}_{2} \mathrm{O}_{2}+\mathrm{H}_{3} \mathrm{O}^{+}+\mathrm{H}_{2} \text { (3) }
$$

Among the species formed during radiolysis of water, it is important to differentiate between reducing and oxidizing species. Reducing species such as $\mathrm{e}_{\mathrm{aq}}^{-}$and $\mathrm{H} \bullet$ will reduce the metal ions in solution to atoms that eventually will coalesce and form nanoparticles, whereas species such as $\mathrm{OH} \bullet$ will oxidize the ions present in the solution, bringing them to a higher valence state [30]. In order to prevent these oxidation processes, scavengers such as isopropanol are added to the reaction mixture [40]. Oxidizing species such as $\mathrm{OH} \bullet$ and reducing species such as $\mathrm{H} \bullet$ interact with isopropanol creating secondary strong reducing radicals [52]. The amount of these reducing and oxidizing species is strongly correlated with the absorbed dose by the aqueous solution. In general, a higher absorbed dose implies an increase in reactive species. Table 3.1, shows the concentration of species formed during radiolysis of water and its variation with increasing absorbed dose.

Table 3.1. The concentration of reactive species generated during radiolysis of water regarding the absorbed dose [135].

\begin{tabular}{|c|c|c|c|c|}
\hline \multirow{2}{*}{$\begin{array}{c}\text { Absorbed } \\
\text { Dose (kGy) }\end{array}$} & \multicolumn{4}{|c|}{ Concentration of species $(\mathbf{m M})$} \\
\cline { 2 - 5 } & $\mathbf{e}_{\mathbf{a q}}$ & $\mathbf{H \bullet}$ & $\mathbf{O H} \bullet$ & $\mathbf{H}_{\mathbf{2}} \mathbf{O}_{\mathbf{2}}$ \\
\hline 0.1 & 0.03 & 0.01 & 0.03 & 0.01 \\
\hline 0.5 & 0.14 & 0.03 & 0.14 & 0.04 \\
\hline 1 & 0.27 & 0.06 & 0.28 & 0.07 \\
\hline 5 & 1.35 & 0.3 & 1.4 & 0.35 \\
\hline 10 & 2.7 & 0.6 & 2.8 & 0.7 \\
\hline
\end{tabular}




\subsubsection{Nucleation and growth of Nanoparticles.}

The processes of nucleation and growth of nanoparticles initiate with the reduction of the metal ions in solution by species such as $\mathrm{e}^{-}$aq and $\mathrm{H} \bullet$. These species have strong reducing potential of $\mathrm{E}^{\circ}\left(\mathrm{H}_{2} \mathrm{O} / \mathrm{e}^{-}{ }_{\text {aq }}\right)=-2.87 \mathrm{~V}_{\mathrm{NHE}}$ and $\mathrm{E}^{\circ}\left(\mathrm{H}^{+} / \mathrm{H}\right)=-2.3 \mathrm{~V}_{\mathrm{NHE}}$ [30]. The energy deposited by the photon source is uniformly deposited in the solution, leading to a homogeneous distribution of radicals and consequently metallic seeds. The process of radiolytic reduction is shown in equation (4) and (5), where $\mathrm{M}^{+}$is the monovalent ion and $\mathrm{M}^{0}$ are zero-valent metal ion [30]. Similarly, multivalent ions are reduced by multistep reactions, by the interaction of the different reactive species within the solutions.

$$
\begin{gathered}
M^{+}+e^{-} a q \rightarrow M^{0}(4) \\
M^{+}+\mathrm{H} \bullet \rightarrow M^{0}+H^{+}(5)
\end{gathered}
$$

The atoms formed within the solution can either dimerize or interact with the remaining metal precursor ions, as seen in equation (6) and (7). Finally, by a multi-step process, these species progressively coalesce and form nucleation centers following equation (8), (9) and (10), with m, $\mathrm{n}$, and $\mathrm{p}$ being the nuclearities and $\mathrm{x}, \mathrm{y}$ and $\mathrm{z}$, are the number of associated ions [30,35]. The redox potential of the clusters increases with the number of atoms in the nucleation center, leading to a rapid coalesce process. The competition of the reduction of free metal and absorbed ions is controlled by the radiation dose [53].

$$
\begin{aligned}
& M^{0}+M^{0} \rightarrow M_{2}(6) \\
& M^{0}+M^{+} \rightarrow M_{2}{ }^{+}(7) \\
& M_{2}^{+}+M_{2}^{+} \rightarrow M_{4}^{2+}(8)
\end{aligned}
$$




$$
\begin{gathered}
M_{m}+M^{+} \rightarrow M_{m+1}^{+}(9) \\
M_{m+x}^{x+}+M_{n+}^{y+} \rightarrow M_{p+z}^{Z+}(10)
\end{gathered}
$$

The charged dimer clusters $\left(\mathrm{M}^{2+}\right)$ can be reduced and form centers of nucleation. The competition between the reduction of free metal ions in solution and absorbed ones are controlled by the dose rate and absorbed dose, parameters that affect the formation of reducing species [52,53]. At lower absorbed doses, the reduction of ions absorbed in the clusters favors cluster growth rather than the formation of new centers of nucleation. The final size of the nanoparticle depends on the limitations imposed by the synthesis process, for example, for nanocolloids in solution, the coalescence may be limited by a stabilizing polymeric molecule [30]. The process of reduction, nucleation and growth of nanoparticles in solution by ionizing radiation, is depicted in Figure 3.3.

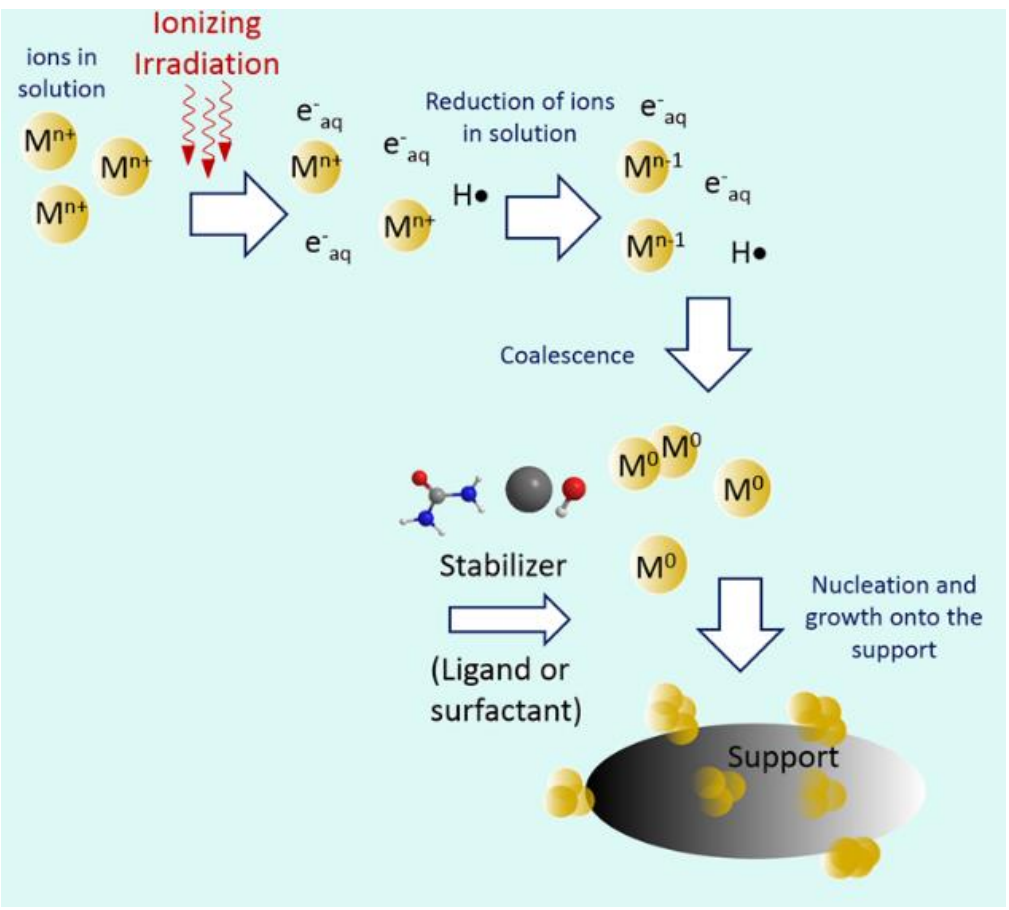

Figure 3.3. Schematic representation of ion reduction by X-ray radiolytic synthesis. 
Nanoparticle formation in radiolytic synthesis follows the classical paths of nanoparticle nucleation and growth. Nanoparticle formation begins when nuclei also known as seeds, act as templates for the crystal to grow. Nucleation can occur through homogeneous or heterogeneous processes. Heterogeneous nucleation takes place when crystals grow within the system such using features such as container surfaces, impurities or seeds intentionally place within solution [51]. In radiolytic synthesis, the nucleation process is through homogeneous processes, where nuclei formation and nanoparticle growth depend on the surface free energy and the bulk free energy [51]. For a spherical particle with a corresponding radius $r$ to form, a maximum free energy needs to be achieved in which the nuclei becomes stable nucleus [51]. The critical radius ( $\left.\mathrm{r}_{\text {crit }}\right)$ is the minimum size that a particle stabilizes and it will not dissolve in solution (equation 11). Where $\mathrm{T}$ is the temperature of the solution, $k_{B}$ is the Boltzmann's constant, $\mathrm{S}$ is the supersaturation of the solution, $v$ is the molar volume and $\gamma$ is the surface energy. The critical radius has a corresponding particle's free energy, where a critical free energy is required to obtain stable particles within solution (equation 12).

$$
\begin{gathered}
r_{c r i t}=-\frac{2}{\Delta G_{v}}=\frac{2 \gamma v}{k_{B} T \ln S}(11) \\
\Delta G_{\text {crit }}=\frac{4}{3} \pi \gamma{r_{\text {crit }}}^{2}(12)
\end{gathered}
$$

The process of reduction of ions in solution relies on the number of reducing species produced by radiolysis processes. Thus, being $\mathrm{OH}^{\bullet}$ and $\mathrm{H}_{2} \mathrm{O}_{2}$ oxidizing species, scavengers are added during the synthesis process [30]. Among various possible molecules, preferred choices are solvents who are unable to oxidize the metal ions. Secondary alcohols interact with oxidizing species such as $\mathrm{OH}^{\bullet}$ through reactions (13) and (14) producing species such as $\left(\mathrm{CH}_{3}\right)_{2} \mathrm{COH}$ and $\mathrm{COO}^{*}$, that act as metal reducing agents [30]. 


$$
\begin{aligned}
\left(\mathrm{CH}_{3}\right)_{2} \mathrm{CHOH}+\mathrm{OH}^{\bullet} & \rightarrow\left(\mathrm{CH}_{3}\right)_{2} \dot{\mathrm{COH}}+\mathrm{H}_{2} \mathrm{O} \\
\mathrm{HCOO}^{-}+\mathrm{OH}^{\bullet} & \rightarrow \mathrm{COO}^{\bullet-}+\mathrm{H}_{2} \mathrm{O}(14)
\end{aligned}
$$

$\mathrm{H}^{*}$ radicals are reducing agents also scavenged by these molecules, to form more reducing species, as shown in equations (15) and (16). These radicals have strong reducing potentials of $\mathrm{E}^{\circ}\left(\left(\mathrm{CH}_{3}\right)_{2} \mathrm{CO} /\left(\mathrm{CH}_{3}\right)_{2} \dot{\mathrm{C}} \mathrm{OH}\right)=-1.8 \mathrm{~V}_{\mathrm{NHE}}$ and $\mathrm{E}^{\circ}\left(\mathrm{CO}_{2} / \mathrm{COO}^{\bullet-}=-1.9 \mathrm{~V}_{\mathrm{NHE}}[30]\right.$.

$$
\begin{gathered}
\left(\mathrm{CH}_{3}\right)_{2} \mathrm{CHOH}+\mathrm{H}^{\bullet} \rightarrow\left(\mathrm{CH}_{3}\right)_{2} \dot{\mathrm{COH}}+\mathrm{H}_{2}(15) \\
\mathrm{HCOO}^{-}+\mathrm{H}^{\bullet} \rightarrow \mathrm{COO}^{\bullet-}+\mathrm{H}_{2}(16)
\end{gathered}
$$

The formation of nanoparticles with radiolytic synthesis is highly dependent on the chemical reduction paths followed by the metallic precursors in solution. Table 3.2 shows the reduction potential of some metal nanoparticles used in nanocomposites systems.

Table 3.2. Standard reduction potential values of some metals [136]

\begin{tabular}{|c|c|}
\hline Electrode Reaction & Potential E $\left(\mathbf{V}_{\text {nhe }}\right)$ \\
\hline$A u^{+}+e^{-} \leftrightarrow A u$ & 1.692 \\
\hline$A u^{3+}+2 e^{-} \leftrightarrow A u^{+}$ & 1.401 \\
\hline$A u^{3+}+3 e^{-} \leftrightarrow A u$ & 1.498 \\
\hline$A u^{2+}+e^{-} \leftrightarrow A u^{+}$ & 1.8 \\
\hline$A u C l_{4}^{-}+3 e^{-} \leftrightarrow A u+4 C l^{-}$ & 1.002 \\
\hline$A g^{2+}+e^{-} \leftrightarrow A g$ & 1.980 \\
\hline$A g^{+}+e^{-} \leftrightarrow A g$ & 0.7996 \\
\hline$C u^{+}+e^{-} \leftrightarrow C u$ & 0.521 \\
\hline$C u^{2+}+e^{-} \leftrightarrow C u^{+}$ & 0.153 \\
\hline$C u^{3+}+e^{-} \leftrightarrow C u^{2+}$ & 2.4 \\
\hline$N i^{2+}+2 e^{-} \leftrightarrow N i$ & -0.257 \\
\hline $\mathrm{NiO}_{2}+4 H^{+}+2 e^{-} \leftrightarrow N i^{2+}+2 \mathrm{H}_{2} O$ & 1.678 \\
\hline$P d^{2+}+2 e^{-} \leftrightarrow P d$ & 0.951 \\
\hline$\left[\mathrm{PdCl}_{4}\right]^{2-}+2 e^{-} \leftrightarrow P d+4 \mathrm{Cl}^{-}$ & 0.591 \\
\hline
\end{tabular}

\subsubsection{Influence of radiation dose.}

The nucleation and growth processes are related to the absorbed dose during the synthesis process.

The rates of growth are determined either by the collisions between atoms, the interaction of one 
atom or ion and nuclei and collision between several nuclei [137]. At low radiation doses, the concentration of metal ions is higher than the nuclei concentration. Thus, the metal ions will aggregate to form nanoparticles with a large diameter [39]. On the other hand, at high radiation doses, most of the metal ions are consumed during the nucleation process, forming a high number of nucleation centers. As a result, nanoparticles with small particle size and narrow particle size distribution are formed [138].

\subsubsection{Stabilization.}

Nanomaterials have high surface energy due to their large surface area, therefore, nanoparticle synthesis is a challenging process, since particles tend to aggregate. Nanoparticles in liquid or colloidal suspensions are attracted to each other by the van der Waals forces. If there is no counteracting force, the particles will aggregate and the colloidal system will be destabilized [53]. Nanoparticle stability is attained when the repulsion and attraction forces are balanced due to electrostatic or steric stabilization. There are several ways to stabilize nanoparticles, which depend on the surface chemistry of metal, the charge of the nanomaterial, and the application of the assynthesized nanoparticles [53]. Compounds such as polymers with functional groups such as $\mathrm{NH} 2$, $-\mathrm{COOH}$, and $-\mathrm{OH}$ have a high affinity for metal atoms, however, the use of polymeric stabilizers could compromise their use in the field of medicine and catalysis. One of the most used polymeric stabilizer for metal nanoparticle synthesis is polyvinylpyrrolidone (PVP) [53]. Due to functional groups such as $\mathrm{C}=\mathrm{O}$ and $\mathrm{N}$ bonds, $\mathrm{PVP}$ can easily bond with the metal nanoparticle surface. Another way to improve nanoparticle aggregation is the combined use of support and ligands. Ligands are molecules or ions with a lone pair of electrons that attach to other ions to form complexes. The synthesis method of deposition-precipitation of $\mathrm{Au}$ supported $\mathrm{TiO}_{2} \mathrm{NCs}$, uses ligands such as sodium hydroxide $(\mathrm{NaOH})$ and urea to produce well-dispersed $\mathrm{Au}$ nanoparticles 
and ensure a strong electrostatic interaction between the metal and the support [39]. For example, when the metal precursor $\left(\mathrm{HAuCl}_{3}\right)$ reacts with $\mathrm{NaOH}$ at a heating temperature of $80^{\circ} \mathrm{C}, \mathrm{NaOH}$ act as a ligand by enabling the bonding of $\mathrm{Au}$ ions to the surface of $\mathrm{TiO}_{2}$ [39].

\subsubsection{Effect of precursor's concentration.}

The final nanoparticle size is dependent upon the initial ion concentration in solution, which applies to chemical and radiolytic synthesis $[53,139]$. A high concentration of metal precursor could lead to the production of large metal nanoparticles [139]. A higher precursor concentration causes a high rate of ion association at the nuclei formation stage, this could cause the formation of large particles [139]. Furthermore, a high concentration of precursor could lead to particle aggregation. It is important to mention that there are strategies to avoid particle aggregation or excessive nanoparticle growth. One of these strategies is the use of stabilizing agents, these agents modify particle movement, preventing aggregation [53]. 


\section{Chapter 4: X-ray radiolytic synthesis of gold supported nanoparticles}

\subsection{Introduction.}

Metal and metal oxide nanoparticles have recently become of great interest to the scientific community because of their potential as catalysts, photosensitizers, and radiosensitizers. $\mathrm{Au} @ \mathrm{TiO}_{2}$ nanocomposites in the field of photocatalysis, have shown an outstanding catalytic activity especially when Au particles of 2-3 nm are dispersed onto supports. In this chapter, an alternative method to synthesize Au nanoparticles onto anatase $\mathrm{TiO}_{2}$ using Xray was thoroughly investigated. This approach is implemented at ambient temperature and pressure and it eliminates the need for harsh chemicals and reducing agents. $\mathrm{Au} @ \mathrm{TiO}_{2}$ nanocomposites were fabricated using absorbed doses ranging from $120 \mathrm{~Gy}$ to $7600 \mathrm{~Gy}$, in the presence different ligands such as $\mathrm{NaOH}$ and urea, and supported by 6.5 or $21.6 \mathrm{~nm}$ titania, to determine their influence of on the Au particle formation, particle size distribution, and loading. Results showed that large absorbed doses control Au nanoparticle size by generating a high of reducing species resulting in an increase of Au seeds onto the titania support. X-ray radiolytic synthesis produced well dispersed Au nanoparticles, a feature that increases the active sites on nanocomposites.

\subsection{Experimental Procedure.}

\subsubsection{Materials and Methods.}

Chloroauric acid $\left(\mathrm{HAuCl}_{4} \cdot 3 \mathrm{H}_{2} \mathrm{O}, \geq 99.9 \%\right.$ trace metal basis $)$, isopropanol $\left(\mathrm{C}_{3} \mathrm{H}_{8} \mathrm{O}, \geq 99.7 \%\right)$, urea and $\mathrm{NaOH}$ were obtained from Sigma-Aldrich. Anatase $\left(\mathrm{TiO}_{2}\right)$, as nanoparticle supports, with a mean particle size of $6.5 \mathrm{~nm}(99.8 \%)$ and $21.6 \mathrm{~nm}(99.0 \%)$ were both obtained from Nanostructured and Amorphous Materials Inc. Deionized water (DI) (18 M $\Omega$ ) was obtained from a Millipore Direct QTM 3 UV purification system and was used to prepare all aqueous solutions. 


\subsubsection{Au ion Deposition onto $\mathrm{TiO}_{2}$ in the presence of $\mathrm{NaOH}$.}

The deposition of $\mathrm{Au}$ onto $\mathrm{TiO}_{2}$ was carried out in amber glassware since light can decompose metallic ions in solution [29]. Initially, a precursor stock solution of $\mathrm{HAuCl}_{4}(2 \mathrm{mM})$ was prepared and the $\mathrm{pH}$ was adjusted to 8 using a $\mathrm{NaOH}$ solution with a concentration of $1 \mathrm{M}$. Subsequently, $4 \mathrm{mg}$ of $\mathrm{TiO}_{2}$ per $\mathrm{ml}$ of stock solution, with either a particle size of 6.5 or $21.6 \mathrm{~nm}$, was added to the $\mathrm{HAuCl}_{4}$. The solution was sonicated for 5 minutes using an ultrasonic probe in order to obtain a homogeneous dispersion of the support in solution. After sonication, the $\mathrm{pH}$ was re-adjusted to a value of 8 using a $\mathrm{NaOH}$ solution $(1 \mathrm{M})$. The solution was then placed on a magnetic hot plate for subsequent heating and stirring for 1 hour at $80{ }^{\circ} \mathrm{C}$. Once the heating process was completed the solutions were centrifuged at $9000 \mathrm{rpm}$ for 10 minutes, decanted and washed with DI water, in order to remove unreactive species. It is important to notice that the $\mathrm{TiO}_{2}$ support and $\mathrm{Au}$ ion concentrations were chosen to give a $10 \%$ loading of Au nanoparticles (by mass) onto the $\mathrm{TiO}_{2}$ supports.

\subsubsection{Au ion Deposition onto $\mathrm{TiO}_{2}$ in the presence of Urea.}

Au ion deposition with urea was also performed in amber glassware. Herein, urea was added to a $2 \mathrm{mM}$ solution of $\mathrm{HAuCl}_{4}$ to give a concentration $0.42 \mathrm{M}$. Subsequently, $\mathrm{TiO}_{2}$ with either a particle size of 6.5 or $21.6 \mathrm{~nm}$ was added at a concentration $4 \mathrm{mg} / \mathrm{ml}$, to ensure a $10 \%$ nominal Au loading. The solution was sonicated with an ultrasonic probe for 5 minutes, followed by heating and magnetic stirring at $80^{\circ} \mathrm{C}$ for $4 \mathrm{hrs}$. The solutions were centrifuged at $9000 \mathrm{rpm}$ for $\sim 10$ minutes, decanted and washed with DI water, in order to remove unreactive species, the washing process was repeated three times.

Au deposition process using either $\mathrm{NaOH}$ or urea as a ligand is an important synthesis step since it allows for the formation of incipient bonds between the $\mathrm{TiO}_{2}$ and $\mathrm{Au}$ complexes within the 
solution. Furthermore, the ligands ensure that the $\mathrm{Au}$ nanoparticles nucleate and grow onto the $\mathrm{TiO}_{2}$ during the irradiation process[46]. The experimental procedure of $\mathrm{Au}$ ion deposition is shown in Figure 4.1.

\section{Mixing of $\mathrm{HAuCl}_{4}$ and $\mathrm{TiO}_{2}$}

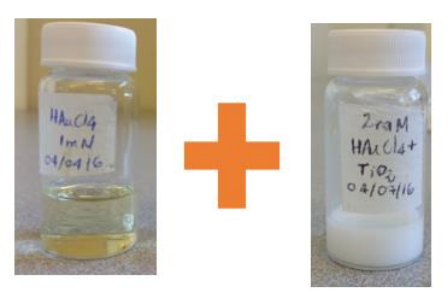

Heating at $80^{\circ} \mathrm{C}$ for 1 hour $(\mathrm{NaOH})$ And 4 hours for Urea

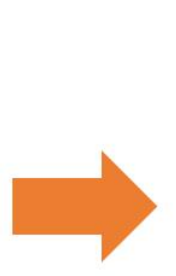

Electrostatic interaction of $\mathrm{TiO}_{2}$ and $\mathrm{Au}$ ions
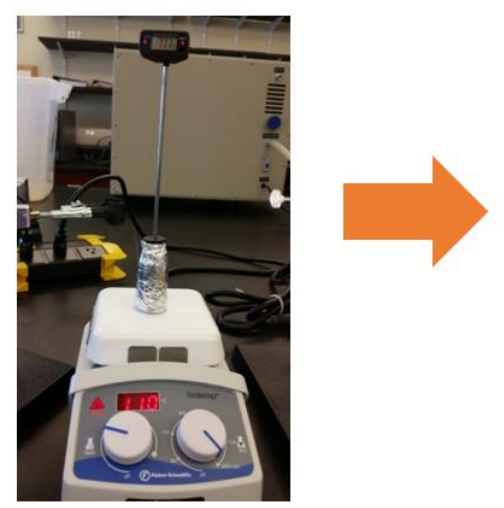

Figure 4.1. $\mathrm{Au}$ ion deposition on $\mathrm{TiO}_{2}$ using $\mathrm{NaOH}$ and Urea.

\subsubsection{Au@ $\mathrm{TiO}_{2}$ Nanocomposites by X-ray Radiolysis.}

After the heating process and before irradiation, isopropanol (IPA) in a 10\% vol/vol, was added to each reaction solution in order to scavenge oxidants formed during radiolysis of water. Following the addition of the scavenger, each reaction solution turned an opaque gray color. The solution was then transferred from amber vials to clear $1.5 \mathrm{ml}$ polypropylene (PP) vials. For irradiation purposes, two X-ray generators were used. The first one was a Panalytical X'Pert Pro X-ray diffractometer equipped with a $\mathrm{Cu}$ target operated at $45 \mathrm{kV}$ and $40 \mathrm{~mA}$ at a dose rate of $4 \mathrm{~Gy} / \mathrm{min}$. Using this device, the solutions were either irradiated for $30,60,120$ or 180 minutes in order to achieve absorbed doses of 120, 240, 480 or 720 Gy respectively. The second X-ray irradiator was an X-RAD 225XL equipped with a W-target operated at $225 \mathrm{kV}$ and $13.3 \mathrm{~mA}$. Samples were irradiated for 60 minutes giving an absorbed dose of $7260 \mathrm{~Gy}$. The absorbed doses were verified in all cases using a Fricke dosimeter. A Fricke dosimeter measures the dose delivered to an aqueous 
solution through the oxidation of $\mathrm{Fe}^{2+}$ into $\mathrm{Fe}^{3+}$ by the reactive species produced in the radiolysis of water. This solution is composed of $0.001 \mathrm{M} \mathrm{FeSO}_{4}, 0.8 \mathrm{~N}$ of $\mathrm{H}_{2} \mathrm{SO}_{4}$ and $0.001 \mathrm{M}$ of $\mathrm{NaCl}$. [17]. After irradiation, the solutions containing the synthesized $\mathrm{Au} @ \mathrm{TiO}_{2}$ nanocomposites solutions were washed with DI water using a centrifuge at $9000 \mathrm{rpm}$ for 10 minutes, the water containing unreactive species was replaced with fresh DI water. Based on the $\mathrm{TiO}_{2}$ support particle size and ligand type in the synthesis, the solutions were named using the form $\mathrm{Au} @ \mathrm{TiO}_{2}\left(\mathrm{size} \mathrm{of}_{\mathrm{TiO}_{2}}\right)^{-}$ ligand to give $\mathrm{Au} @ \mathrm{TiO}_{2}{ }^{(6.5 \mathrm{~nm})-\mathrm{NaOH}}, \mathrm{Au} @ \mathrm{TiO}_{2}{ }^{(6.5 \mathrm{~nm})-\mathrm{urea}}, \mathrm{Au} @ \mathrm{TiO}_{2}{ }^{(21.6 \mathrm{~nm})-\mathrm{NaOH}}$ and $\mathrm{Au} @ \mathrm{TiO}_{2}{ }^{(21.6 n m)-}$ urea. For radiosensitization experiments, $\mathrm{Au} @ \mathrm{TiO}_{2}$ samples were dried in an oven at $40{ }^{\circ} \mathrm{C}$ for several days. Then, the obtained purple pellet was ground into a fine powder using a mortar and pestle. After irradiation, the obtained powders and solutions were stored in the dark. The overall experimental procedure is shown in figure 4.2 .

1. Mixing of the aqueous Solutions

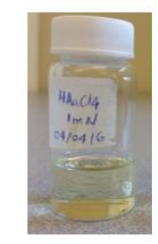

$\mathrm{HAuCl}_{4}+\mathrm{NaOH}$

3. X Ray irradiation

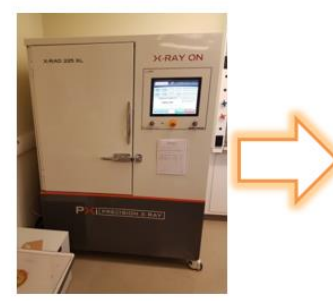

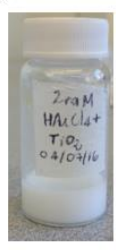

$\mathrm{TiO}_{2}$

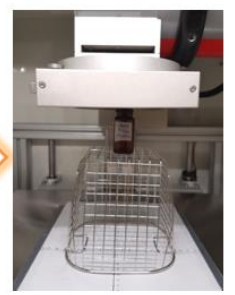

2. Heating of the solution for 1 hour at $80^{\circ} \mathrm{C}$
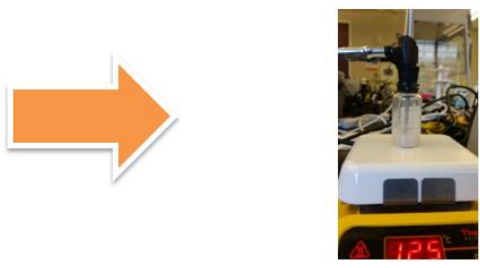

4. Synthesis of TiO2/Au nanoparticles

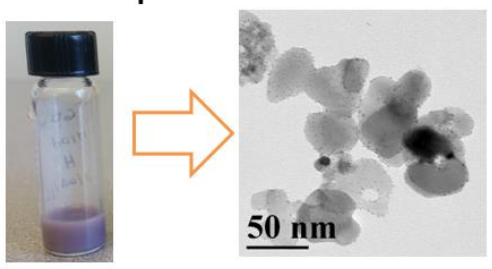

Figure 4.2. Experimental Procedure of X-ray radiolytic synthesis of $\mathrm{Au} @ \mathrm{TiO}_{2}$ nanocomposites.

\subsubsection{Characterization of $\mathrm{Au} @ \mathrm{TiO}_{2}$ Nanocomposites.}

Particle size and particle size distribution of the supported Au nanoparticles were investigated by TEM using a Zeiss Libra 120 operated at $120 \mathrm{KV}$ and an FEI Titan 300, operated at $300 \mathrm{keV}$ for 
high-resolution imaging (HR-TEM). For TEM analysis, the $\mathrm{Au} @ \mathrm{TiO}_{2}$ solution was diluted 50 times in acetone followed by sonication for 5 minutes. A drop of each suspension was deposited onto a formvar-carbon copper grid (mesh 300 ) and allowed to dry at room temperature. The growth of Au nanoparticles was qualitatively evaluated using a UV-Vis spectrophotometer GENESYS 10S. The analysis was carried out evaluating the evolution of Au surface plasmon peak of the samples irradiated at different times and at a dose rate of $127 \mathrm{~Gy} / \mathrm{min}$. Furthermore, the crystalline structure of the synthesized $\mathrm{Au} @ \mathrm{TiO}_{2}$ nanocomposites was investigated using a Panalytical X'Pert Pro X-ray diffractometer equipped with a $\mathrm{Cu} X$-ray target (voltage $45 \mathrm{kV}$ and current $40 \mathrm{~mA}$ ). The $\mathrm{Au} @ \mathrm{TiO}_{2}$ nanocomposites powders were prepared by drying aqueous suspension under vacuum at $40^{\circ} \mathrm{C}$. The dried powder was carefully placed onto a low background oriented silicon wafer substrate and mounted onto a $360^{\circ}$ spinner stage with angular velocity kept at $120 \mathrm{rpm}$. The surface chemistry of the synthesized $\mathrm{Au} @ \mathrm{TiO}_{2}$ nanocomposites was carried out using X-ray photoelectron spectroscopy (XPS) (ESCALab 250 X-ray photoelectron spectrophotometer, with a monochromatic Al target X-ray source). The XPS samples were prepared by depositing a drop of the solution onto a Si wafer and allowed to dry. Finally, ICP-OES analysis was made in order to quantify the concentration of $\mathrm{Au}$ supported on the $\mathrm{TiO}_{2}$. Analysis of loading and decoration of $\mathrm{Au} @ \mathrm{TiO}_{2}$ nanocomposites at an absorbed dose of 7260 Gy was made with ICP-OES using a Varian

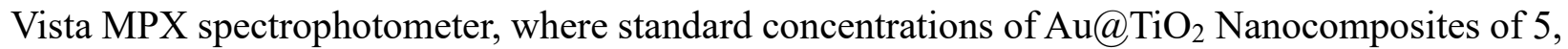
50,100 and $250 \mathrm{ppm}$ were dissolved in aqua regia in order to measure the $\mathrm{Au}$ concentration deposited onto the $\mathrm{TiO}_{2}$ support The sample preparation for the different characterization techniques is shown in Figure 4.3. 

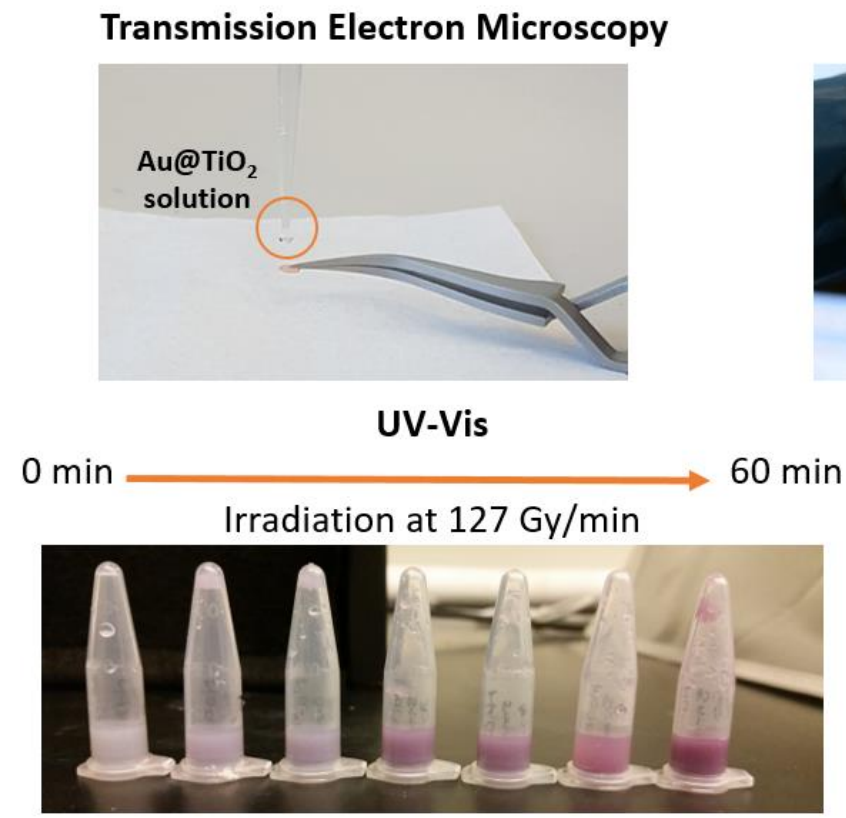

X-Ray Diffraction

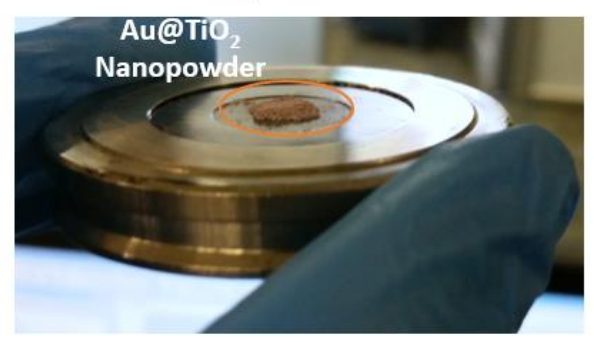

X-Ray Photoelectron Spectroscopy

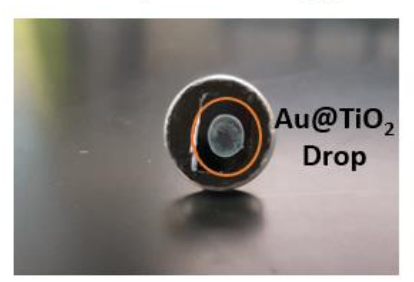

Figure 4.3. Sample Preparation of the different Characterization techniques.

\subsection{Results and Discussion.}

\subsubsection{Crystal Structure of $\mathrm{Au} @ \mathrm{TiO}_{2}$ Nanocomposites.}

The crystalline structure of the $\mathrm{Au} @ \mathrm{TiO}_{2}$ nanocomposites was evaluated with XRD. The patterns of both $\mathrm{TiO}_{2}$ supports were verified to be of an anatase phase (pdf: 01-075-2552). A mean particle size estimation was made using the Scherrer equation, revealing crystallite sizes of $18.6 \mathrm{~nm}$ and $7.1 \mathrm{~nm}$ for $\mathrm{TiO}_{2}$ with a nominal mean particle size of 21.6 and $6.5 \mathrm{~nm}$ respectively. Figure 4.4 shows the XRD patterns of Au nanoparticles supported onto either $21.6 \mathrm{~nm}(\mathrm{a}-\mathrm{b})$ or $6.5 \mathrm{~nm}$ (c-d) $\mathrm{TiO}_{2}$ supports with $\mathrm{NaOH}$ or urea as ligands respectively. All patterns of $\mathrm{Au} @ \mathrm{TiO}_{2}$ nanocomposites shown in Figure 4.4 were synthesized using an absorbed dose of 7260 Gy. In Figure 4.4, a shift to lower $2 \theta$ of $\mathrm{TiO}_{2}(6.5 \mathrm{~nm})$ was observed when compared with $\mathrm{TiO}_{2}(21.6 \mathrm{~nm})$ which is due to the increase in the lattice parameter [140]. Additionally, the strong intensity of the (101) plane in $\mathrm{TiO}_{2}$ with a mean particle size of $21.6 \mathrm{~nm}$ is caused by the preferred orientation evidenced by the elongated shape of the supports as shown in Figure 4.5a, where a TEM image of 
the support prior to Au deposition is shown. In contrast, the XRD spectra of $6.5 \mathrm{~nm} \mathrm{TiO}_{2}$, show no variations in the relative intensity of the peaks, this correlates with the nearly spherical shape of the supports show in Figure 4.5b. The Au peaks were observed at $2 \theta$ s (degrees) of $44.9,64.8$ and $77.6^{\circ}$ correspond to Au with a face-centered cubic (fcc) structure (pdf: 01-071-4614). When $\mathrm{Au} @ \mathrm{TiO}_{2}{ }^{(21.6 \mathrm{~nm})-\mathrm{NaOH}}$ nanocomposites were evaluated, Scherrer analysis of the peak at a $2 \theta$ position of $64.8^{\circ}$ revealed an $\mathrm{Au}$ average crystallite size of $8.1 \mathrm{~nm}$ and for $\mathrm{Au} @ \mathrm{TiO}_{2}{ }^{(6.5 \mathrm{~nm})-\mathrm{NaOH}}$ gave $8.6 \mathrm{~nm}$. Furthermore, Scherrer Au particle size measurement of $\mathrm{Au} @ \mathrm{TiO}_{2}{ }^{(21.6 \mathrm{~nm}) \text {-urea }}$ gave a mean crystallite size of $12.6 \mathrm{~nm}$ and for $\mathrm{Au} @ \mathrm{TiO}_{2}{ }^{(6.5 \mathrm{~nm}) \text {-urea }}$ a value of $6.8 \mathrm{~nm}$ was found.

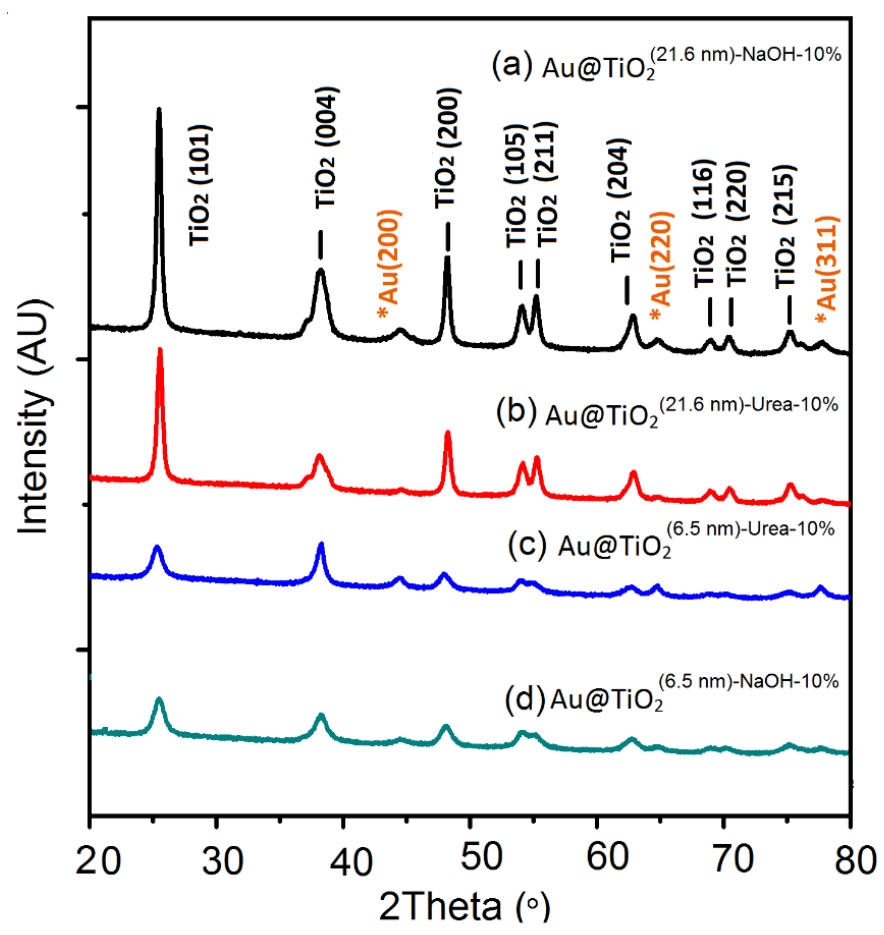

Figure 4.4. XRD Pattern of $\mathrm{Au} @ \mathrm{TiO}_{2}$ nanocomposites synthesized with either $\mathrm{NaOH}$ or urea as ligands and different support sizes at an absorbed dose of $7260 \mathrm{~Gy}$.

\subsubsection{Morphology of $\mathrm{Au} @ \mathrm{TiO}_{2}$ nanocomposites.}

Analysis of TEM images of $\mathrm{TiO}_{2}$ prior Au deposition show $\mathrm{TiO}_{2}$ with a mean particle size of 21.5 $\pm 5.4 \mathrm{~nm}$ and $\mathrm{TiO}_{2}$ with a mean particle size of $6.5 \pm 1.2 \mathrm{~nm}$ are shown in Figure 4.5a and Figure 
4.5b respectively. Analysis of the morphology of $\mathrm{Au}$ supported $\mathrm{TiO}_{2}$ nanoparticles revealed a strong relationship between the Au particle size and loading with the absorbed dose. Figure 4.6a, Figure 4.6b, and Figure 4.6c show $\mathrm{Au} @ \mathrm{TiO}_{2}{ }^{(21.6 n m)-\mathrm{NaOH}}$ synthesized using absorbed doses of 120, 240 and 480 Gy respectively. At lower absorbed doses such as 120 and 240 Gy observed in Figure 4.6a and Figure 4.6b, the mean particle size was almost constant, $5.7 \pm 1.5 \mathrm{~nm}$ and $5.6 \pm 2.2 \mathrm{~nm}$ respectively. Nonetheless, an increase in the number of supported Au nanoparticles was observed at an absorbed dose of $240 \mathrm{~Gy}$. At a dose of $480 \mathrm{~Gy}$ in Figure 4.6c, a mean particle size of $1.7 \pm$ $1.3 \mathrm{~nm}$ was observed. Yet, a few Au particles with sizes varying from 5-9 nm were still obtained. This result indicates that high radiation doses lead to smaller Au nanoparticles on the surface. The mechanism of synthesis of Au nanoparticles on the $\mathrm{TiO}_{2}$ support relies on the interaction of high energy photons with $\mathrm{H}_{2} \mathrm{O}$ molecules leading to the radiolytic cleavage of water. When an aqueous solution is irradiated, the production of reactive species such as $\mathrm{e}_{\mathrm{aq}}^{-}, \mathrm{H} \bullet$ promotes the reduction of metal ions in solution to a zero valence state $[30,35,53]$. Nonetheless, $\mathrm{OH} \bullet$ radicals are oxidizing species that bring the metal ions or atoms to a higher valence state. In order to prevent these reoxidation processes, isopropanol was added to the reaction mixture [35]. Oxidizing species such as $\mathrm{OH} \bullet$ and reducing species such as $\mathrm{H} \bullet$ interact with isopropanol creating secondary strong reducing radicals such as $\mathrm{H}_{3} \mathrm{C}-{ }^{-} \mathrm{C}(\mathrm{OH})-\mathrm{CH}_{3}$ and ${ }^{\circ} \mathrm{CO}_{2}^{-}$[52]. Radiolytic reduction provides a homogeneous distribution of the $\mathrm{Au}^{(0)}$ clusters in the solution that will act as seeds in the nanoparticle growth process [141]. The Au particle size difference of $70 \%$ between those synthesized at absorbed doses of 240 Gy and 480 Gy may be attributed to a number of nuclei formed during the radiolysis process. When the reaction is stopped at low absorbed doses such as $240 \mathrm{~Gy}$, the consumption of the Au atoms of ions in the solution contribute to the growth of already formed nanoparticles rather than to the formation of new nuclei, thus the particle size of the 
supported Au increases [51]. In general, the number of nuclei is lower at $120 \mathrm{~Gy}$ and $240 \mathrm{~Gy}$ compared to an absorbed dose of $480 \mathrm{~Gy}$. This shows that the amount of $\mathrm{Au}^{(0)}$ nuclei is controlled by the absorbed dose delivered to the solution. Nuclei formation in Au may follow two pathways. The first one happens when a cluster of Au ions is formed and then intra-particle ripening takes place, whereas the second one occurs when the Au is reduced, Au seeds are formed, and ions in solution will interact with seeds causing subsequent reduction [51]. The mechanism by which Au ions such as $\mathrm{Au}^{(\mathrm{I})}$ and $\mathrm{Au}^{(\mathrm{III})}$ interact with the $\mathrm{Au}^{(0)}$ seeds within the solution is a phenomenon known as autocatalytic reduction [43]. The principal mechanism of autocatalytic reactions is based on $\mathrm{e}^{-}$transfer from the $\mathrm{Au}$ ions to the $\mathrm{Au}^{(0)}$ surface, this process is known to last less than $200 \mathrm{~ms}$ [51].
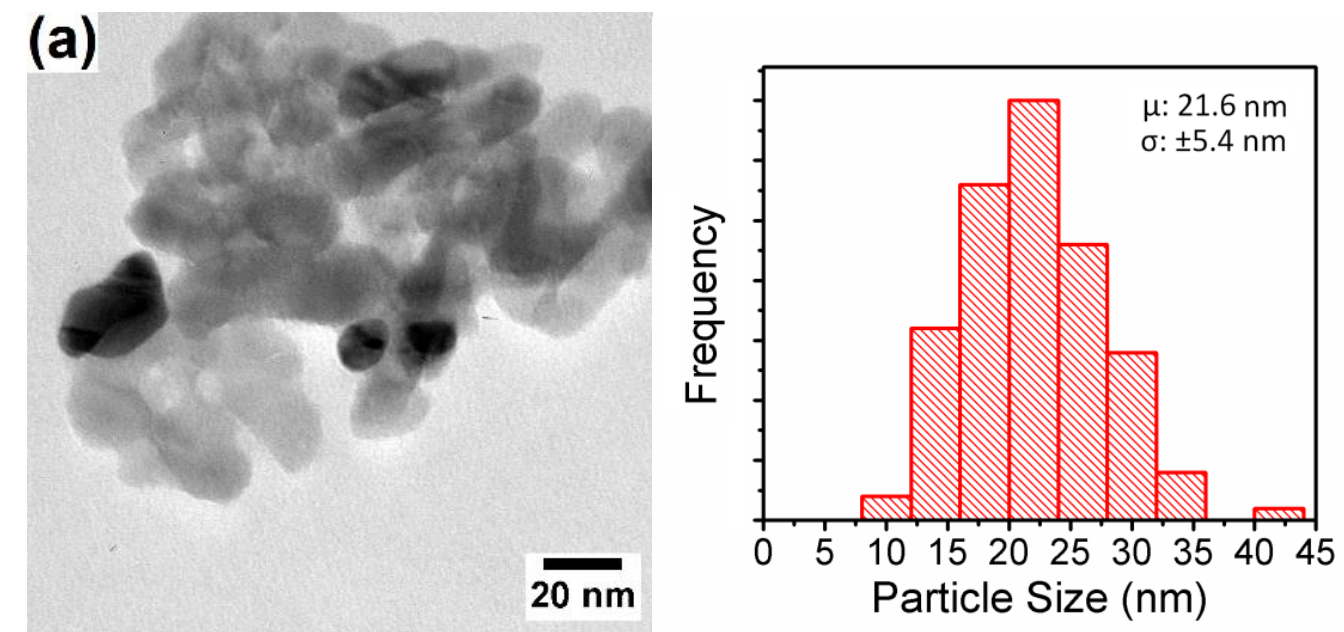

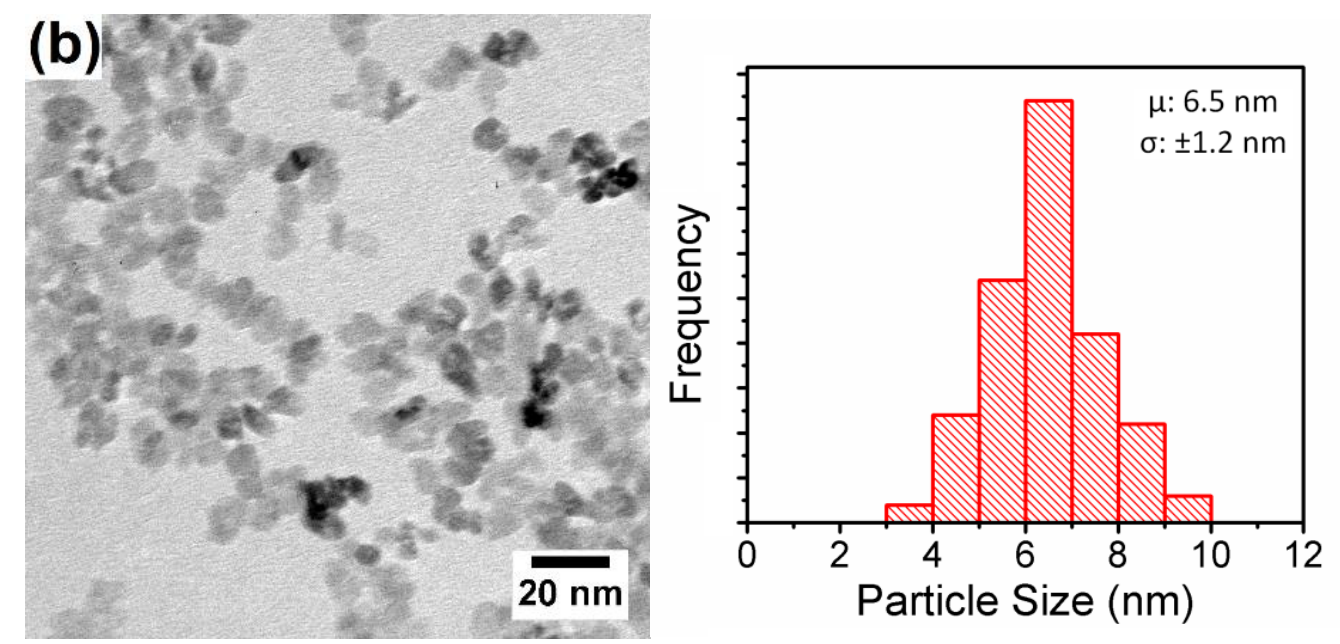

Figure 4.5. TEMs of $\mathrm{TiO}_{2}$ with a particle size distribution of a) $21.6 \mathrm{~nm}$ and b) $6.5 \mathrm{~nm}$. These images were taken prior to irradiation in order to ensure the integrity of the supports before synthesis.
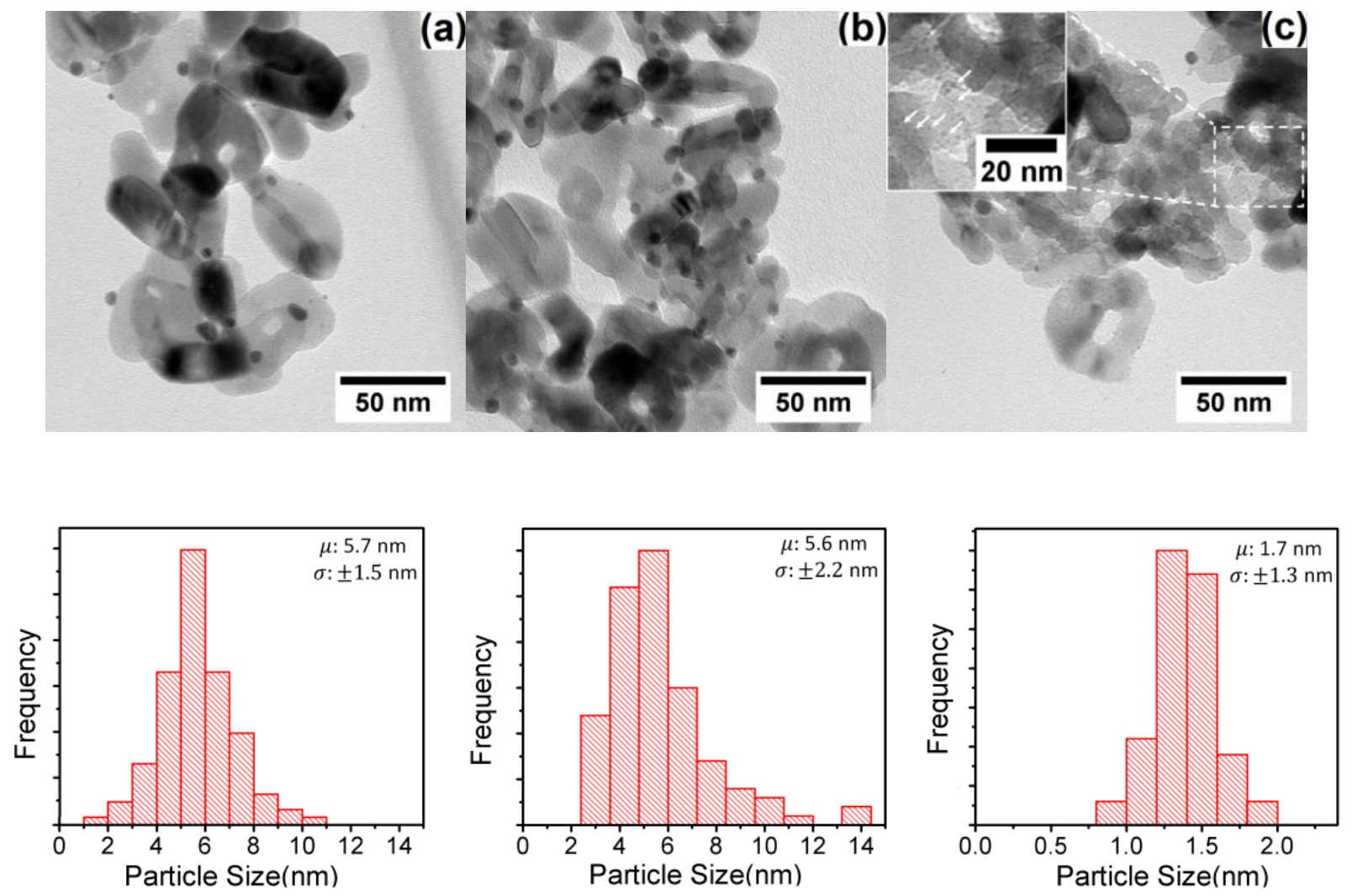

Figure 4.6. TEMs of $\mathrm{Au} @ \mathrm{TiO}_{2}{ }^{(21.6 \mathrm{~nm})-\mathrm{NaOH}}$ nanocomposites with an absorbed dose of a) 120 b) 240 and c) $480 \mathrm{~Gy}$. 
TEM images of the Au nanoparticle size at an absorbed dose of $720 \mathrm{~Gy}$ using either $\mathrm{NaOH}$ or urea as ligands are shown in Figure 4.7a and Figure 4.7b respectively. Selected area electron diffraction (SAED) patterns of the $\mathrm{Au} @ \mathrm{TiO}_{2}$ nanocomposites is given in the inset of Figure 4.7b. The SAED analysis was made in order to verify the crystalline structure of both $\mathrm{Au}$ and $\mathrm{TiO}_{2}$ with the results obtained by XRD. In the SAED patterns, Bragg reflections of planes of Au (fcc), such as (200), (220) and (311) were found. Furthermore, the red circles indicate the titania phase with reflections caused by the planes (101), (004), (200) and (202). These results are in agreement with the X-ray diffraction patterns. Using $\mathrm{NaOH}$ as the ligand a mean particle size of $1.3 \pm 0.3 \mathrm{~nm}$ was found, whereas Au nanoparticles made with urea revealed a slightly larger particle size of $1.6 \pm 0.3 \mathrm{~nm}$. The particle size obtained by TEM is smaller when compared with the size obtained in XRD of $8.1 \mathrm{~nm}$ and $12.6 \mathrm{~nm}$ for Au synthesized by $\mathrm{NaOH}$ and Urea respectively. This is due to the presence of a few Au particles of $\sim 25 \mathrm{~nm}$ as detected by SEM. The morphology of the Au particles is spherical, uniformly distributed nanoparticles onto the titania supports, regardless of ligand treatment or support size.
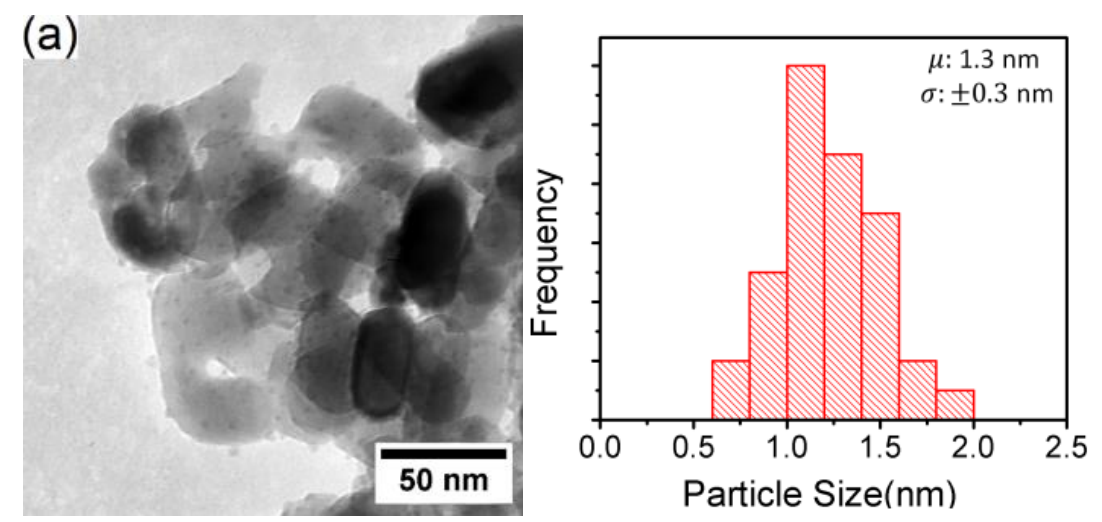

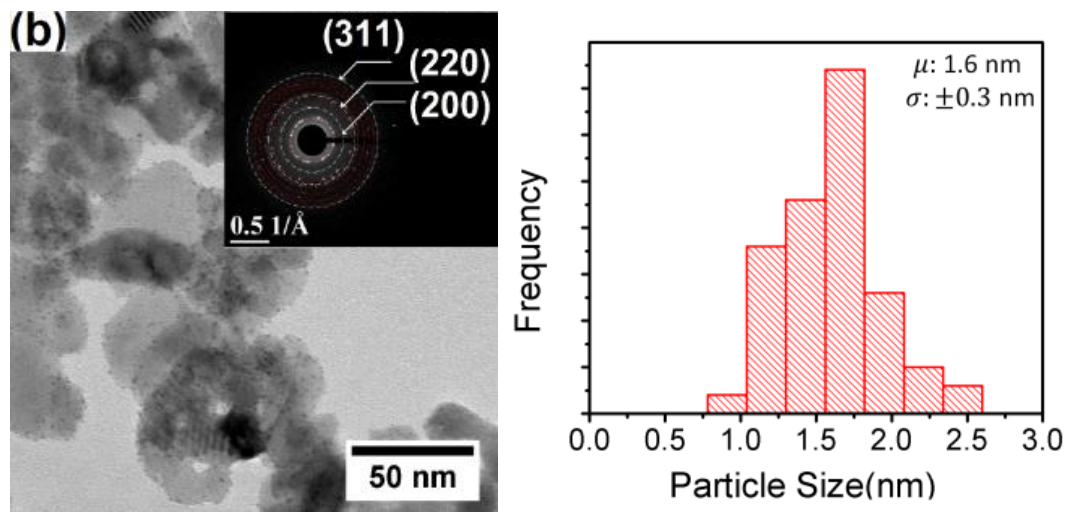

Figure 4.7. TEMs of $\mathrm{Au} @ \mathrm{TiO}_{2}$ nanocomposites at an absorbed dose of 720 Gy for a) $\mathrm{Au} @ \mathrm{TiO}_{2}{ }^{(21.6 \mathrm{~nm})-\mathrm{NaOH}}$, and b) $\mathrm{Au} @ \mathrm{TiO}_{2}{ }^{(21.6 n m)-u r e a}$, with inset of SAED pattern showing $\mathrm{Au}$ reflections by white circles and anatase by red dashed circles.

$\mathrm{Au}$ nanoparticle binding processes to the $\mathrm{TiO}_{2}$, are different depending on the ligand of choosing, this process also impacts the loading of the final nanocomposite $[29,142]$. The analysis of Au nanoparticle loading was made with ICP-OES. Results show that synthesis with urea leads to a higher loading than that with $\mathrm{NaOH}$ by $26.6 \%$ (Table 2.1). The reason behind a loading difference when using urea is due to the fact that most of the $\mathrm{Au}^{(\mathrm{III})}$ species are precipitated during the 4 hours of the heating process. Results from ICP-OES show that the use of $\mathrm{NaOH}$ produce a loading of 7.5 wt $\%$ of $\mathrm{Au}$ onto the surface of the titania, while the use of urea can achieve up to $8 \mathrm{wt} \%$ on the surface of $\mathrm{TiO}_{2}$ for a targeted concentration of $10 \mathrm{wt} \%$ of Au. The difference of loading between $\mathrm{Au} @ \mathrm{TiO}_{2}{ }^{(10-30 \mathrm{~nm})-\mathrm{NaOH}}$ and $\mathrm{Au} @ \mathrm{TiO}_{2}{ }^{(10-30 \mathrm{~nm})-\text { rea }}$ regarding ICP-OES results was $26.6 \%$, where $\mathrm{Au} @ \mathrm{TiO}_{2}{ }^{(10-30 \mathrm{~nm})-\text { urea }}$ was found to provide a better loading. 
Table 4.1. ICP OES of $\mathrm{Au} @ \mathrm{TiO}_{2}$ loading and decoration at a total absorbed dose of $7260 \mathrm{~Gy}$.

Note (bi): before irradiation and (ai): after irradiation.

\begin{tabular}{|c|c|}
\hline Sample & $\begin{array}{c}\text { Loading of } \mathbf{~ A u ~ o n ~} \mathrm{TiO}_{2} \\
\text { (wt\%) }\end{array}$ \\
\hline $\mathrm{Au} @ \mathrm{TiO}_{2}{ }^{(21.6 \mathrm{~nm})-\mathrm{NaOH}}(\mathrm{bi})$ & 9.4 \\
\hline $\mathrm{Au} @ \mathrm{TiO}_{2}{ }^{(21.6 \mathrm{~nm})-\mathrm{NaOH}}(a i)$ & 7.5 \\
\hline $\mathrm{Au} @ \mathrm{TiO}_{2}{ }^{(21.6 \mathrm{~nm})-\text { urea }}(\mathrm{bi})$ & 8.4 \\
\hline $\mathrm{Au} @ \mathrm{TiO}_{2}{ }^{(21.6 \mathrm{~nm})-\text { urea }}(a i)$ & 9.5 \\
\hline
\end{tabular}

During the heating process, the deposition-precipitation method involves the deposition of $\mathrm{Au}^{\text {(III) }}$ and $\mathrm{Au}^{(\mathrm{I})}$ onto the titania surface but the deposition of these species in the presence of $\mathrm{NaOH}$ and urea takes place in different ways [46]. The binding process with $\mathrm{NaOH}$ is due to the hydroxyl groups on the surface of the support by means of either reaction shown in (17) or (18)

$$
\begin{aligned}
& \mathrm{Ti}-\mathrm{OH}+\left[\mathrm{AuCl}_{2}(\mathrm{OH})_{2}\right]^{-} \rightarrow \mathrm{Ti}-\mathrm{O}-\mathrm{Au}(\mathrm{Cl})_{2}+\mathrm{H}_{2} \mathrm{O}+{ }^{-} \mathrm{OH} \\
& \mathrm{Ti}-\mathrm{OH}+\left[\mathrm{AuCl}(\mathrm{OH})_{3}\right]^{-} \rightarrow \text { Ti-O-AuCl}(\mathrm{OH})+\mathrm{H}_{2} \mathrm{O}+2^{-} \mathrm{OH}
\end{aligned}
$$

The reactions in (13) and (14) lead to the formation of a compound only formed by Au-OH species [46]. In contrast, the deposition-precipitation with urea the $\mathrm{Au}^{(\mathrm{III})}$ species deposited onto the support surface make a strong compound containing nitrogen, oxygen, and carbon [46]. The hydrolysis of urea is given in reaction (19)

$$
\mathrm{CO}\left(\mathrm{NH}_{2}\right)_{2}+3 \mathrm{H}_{2} \mathrm{O} \rightarrow 2 \mathrm{NH}_{4}^{+}+\mathrm{CO}_{2}(\mathrm{~g})+2^{-} \mathrm{OH}
$$

The small size and dispersion of the Au nanoparticles fabricated using urea as ligand and observed in Figure 4.7b, arise from a process of segregation and fragmentation of $\mathrm{Au}(\mathrm{OH})_{3}$ during heating [46]. At low $\mathrm{pH}$, large aggregates of incipient seeds precipitate onto the surface of titania, 
afterward, a slow peptization (disaggregation) takes places as the $\mathrm{pH}$ increases and the aggregates are redistributed [46]. As a result of peptization, the number of precipitates when using urea is slightly larger than the number of precipitates found with $\mathrm{NaOH}$. Therefore, the loading by mass of Au nanoparticles is larger when urea is utilized. Overall, the number of $\mathrm{Au}(\mathrm{OH})_{3}$ precipitates onto $\mathrm{TiO}_{2}$ serve as nucleation sites during the heterogeneous nucleation process that takes place when the reducing species produced during $\mathrm{X}$-ray radiolysis reduce the $\mathrm{Au}^{(\mathrm{III})}$ ions. Thermogravimetric analyses of the $\mathrm{Au} @ \mathrm{TiO}_{2}$ nanocomposites fabricated with $\mathrm{NaOH}$ and urea are shown in Figure 4.8. Results indicate that traces of urea were present in the nanocomposite, when urea is heated at $200{ }^{\circ} \mathrm{C}$, decomposes into biuret, cyanuric acid, and isocyanic acid. Biuret decomposes at $250^{\circ} \mathrm{C}$ into isocyanic acid [143].

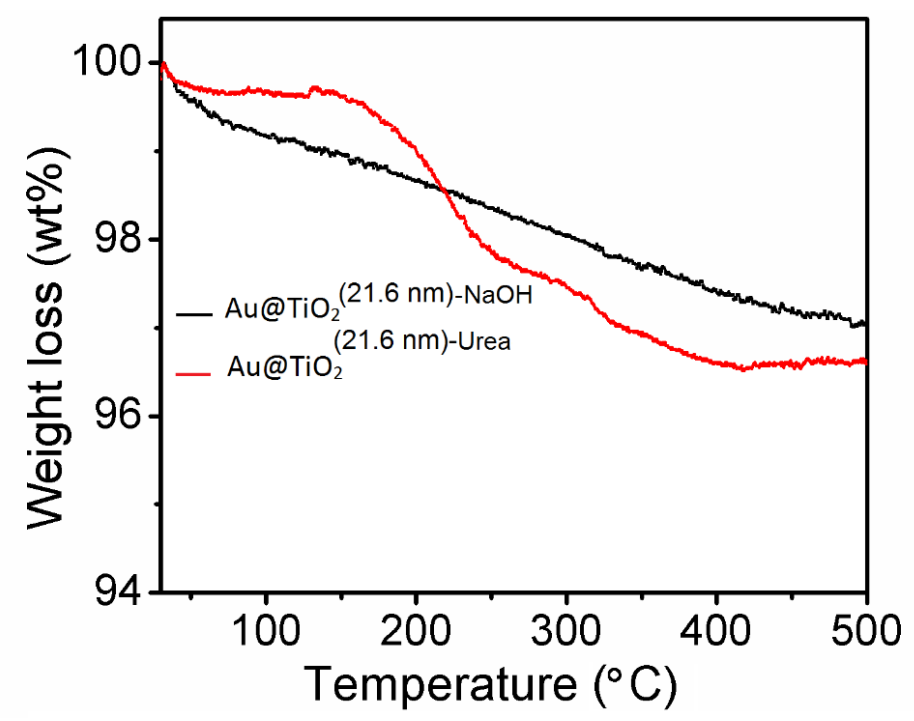

Figure 4.8. TGA analysis of $\mathrm{Au} @ \mathrm{TiO}_{2}$ fabricated with $\mathrm{NaOH}$ and Urea.

The growth and progressive loading of $\mathrm{Au}$ nanoparticles onto $\mathrm{TiO}_{2}$ were followed by the evolution of the Au surface plasmon resonance peak $\sim 545 \mathrm{~nm}$ for $\mathrm{Au} @ \mathrm{TiO}_{2}{ }^{(6.5 \mathrm{~nm})-\mathrm{NaOH}}$ as shown in Figure 4.9a. From the spectra in Figure 4.9a, a shift to lower wavelength is observed as the irradiation time increases. At 10 minutes of irradiation, the Au peak is located at $545 \mathrm{~nm}$, whereas after 60 minutes of irradiation the peak is located at a wavelength of $538 \mathrm{~nm}$. This shifting is due to a 
reduction of the particle size of $\mathrm{Au}$ and an increase in the peak absorbance is related to the higher loading of Au nanoparticles on $\mathrm{TiO}_{2}$. Figure $4.9 \mathrm{~b}$ shows a STEM image of $\mathrm{Au} @ \mathrm{TiO}_{2}{ }^{(6.5 \mathrm{~nm})-\mathrm{NaOH}}$ after 60 minutes of irradiation and an absorbed dose of $7260 \mathrm{~Gy}$. After 60 minutes of irradiation, a particle size of $1.0 \pm 0.2 \mathrm{~nm}$ was found. UV-Vis spectroscopy was also performed for $\mathrm{Au} @ \mathrm{TiO}_{2}{ }^{(21.6 n m)-\mathrm{NaOH}}$, irradiated at various times up to 90 minutes (Figure $4.9 \mathrm{c}$ ), nevertheless, the peak at $\sim 538 \mathrm{~nm}$ did not change in absorbance or position when compared with the peak observed after 60 minutes of sample irradiation. Hence, at an absorbed dose of $7260 \mathrm{~Gy}$ and at the given experimental conditions most of the metallic ions present in the solution were successfully reduced, therefore nanocomposites synthesized at an absorbed dose of 7260 Gy were subsequently analyzed.
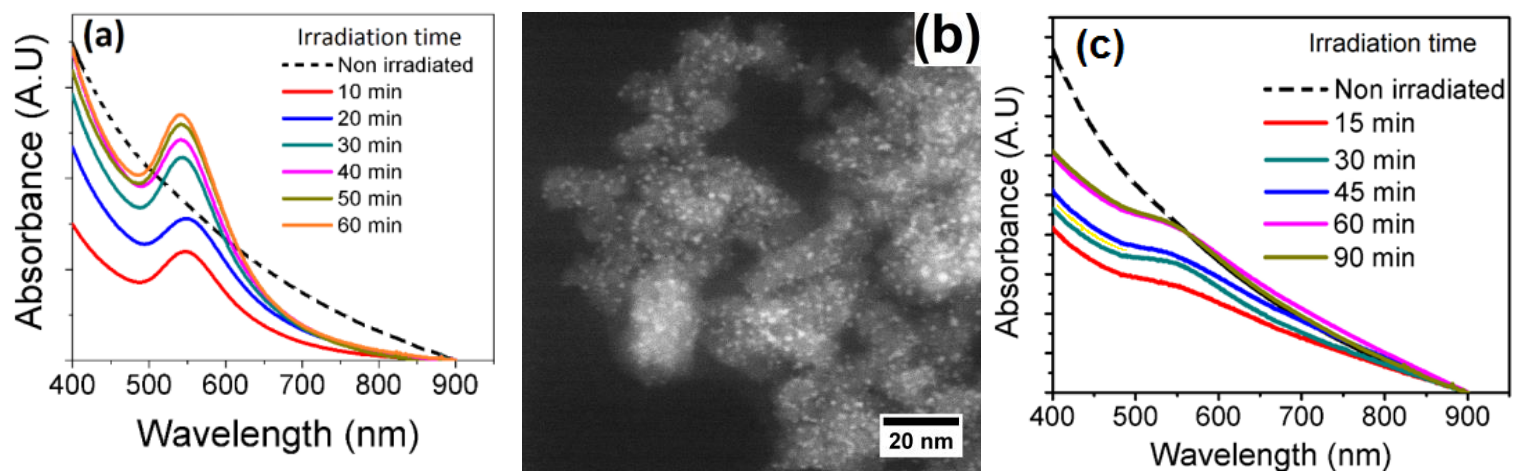

Figure 4.9. a) UV Vis of $\mathrm{Au} @ \mathrm{TiO}_{2}{ }^{(6.5 \mathrm{~nm})-\mathrm{NaOH}}$ nanocomposites and the evolution of Au plasmon resonance at a dose rate of $127 \mathrm{~Gy} / \mathrm{min}$ b) STEM of $\mathrm{Au} @ \mathrm{TiO}_{2}{ }^{(6.5 \mathrm{~nm})-\mathrm{NaOH}}$ at 60 minutes of irradiation time (7260 Gy) c) UV Vis of $\mathrm{Au} @ \mathrm{TiO}_{2}{ }^{(21.6 \mathrm{~nm})-\mathrm{NaOH}}$ nanocomposites and the evolution of Au plasmon resonance up to 90 minutes of irradiation.

The TEM analysis of the Au particle size at an absorbed dose of 7260 Gy using urea onto different particle size supports is shown in Figure 4.10. The d-spacing for both $\mathrm{TiO}_{2}$ and Au nanoparticles in the $\mathrm{Au} @ \mathrm{TiO}_{2}$ nanocomposites was measured with HR-TEM as shown in Figure 4.10a, giving $\mathrm{d}_{(101)}$ of $0.37 \mathrm{~nm}$ and $\mathrm{d}_{(200)}$ of $0.20 \mathrm{~nm}$, respectively. Analysis of the Au particle size at an absorbed 
dose of $7260 \mathrm{~Gy}, \mathrm{Au} @ \mathrm{TiO}_{2}{ }^{(21.6 n m)-u r e a}$ showed in Figure 4.10a, gave a mean particle size of $1.1 \pm$ $0.2 \mathrm{~nm}$. Similarly, Au particle size analysis of $\mathrm{Au} @ \mathrm{TiO}_{2}{ }^{(6.5 \mathrm{~nm})-\text { urea }}$, in Figure $4.10 \mathrm{~b}$, gave a particle size of $1.2 \pm 0.2 \mathrm{~nm}$. It was found that a mean particle size of the Au nanoparticles obtained at $7260 \mathrm{~Gy}$ is smaller as compared to those obtained at a dose of $720 \mathrm{~Gy}$. In Figure 4.10, it is observed that increased absorbed doses generate smaller Au nanoparticles and provide better loading onto the titania surface than the absorbed dose of 720 Gy shown in Figure 4.7. Overall, nanocomposites fabricated at intermediate ( $720 \mathrm{~Gy}$ ) and high absorbed doses ( $7260 \mathrm{~Gy}$ ) show narrow particle size distribution, high Au loading, and produces homogeneously dispersed particles onto the support, valuable features for catalytical and medical applications.
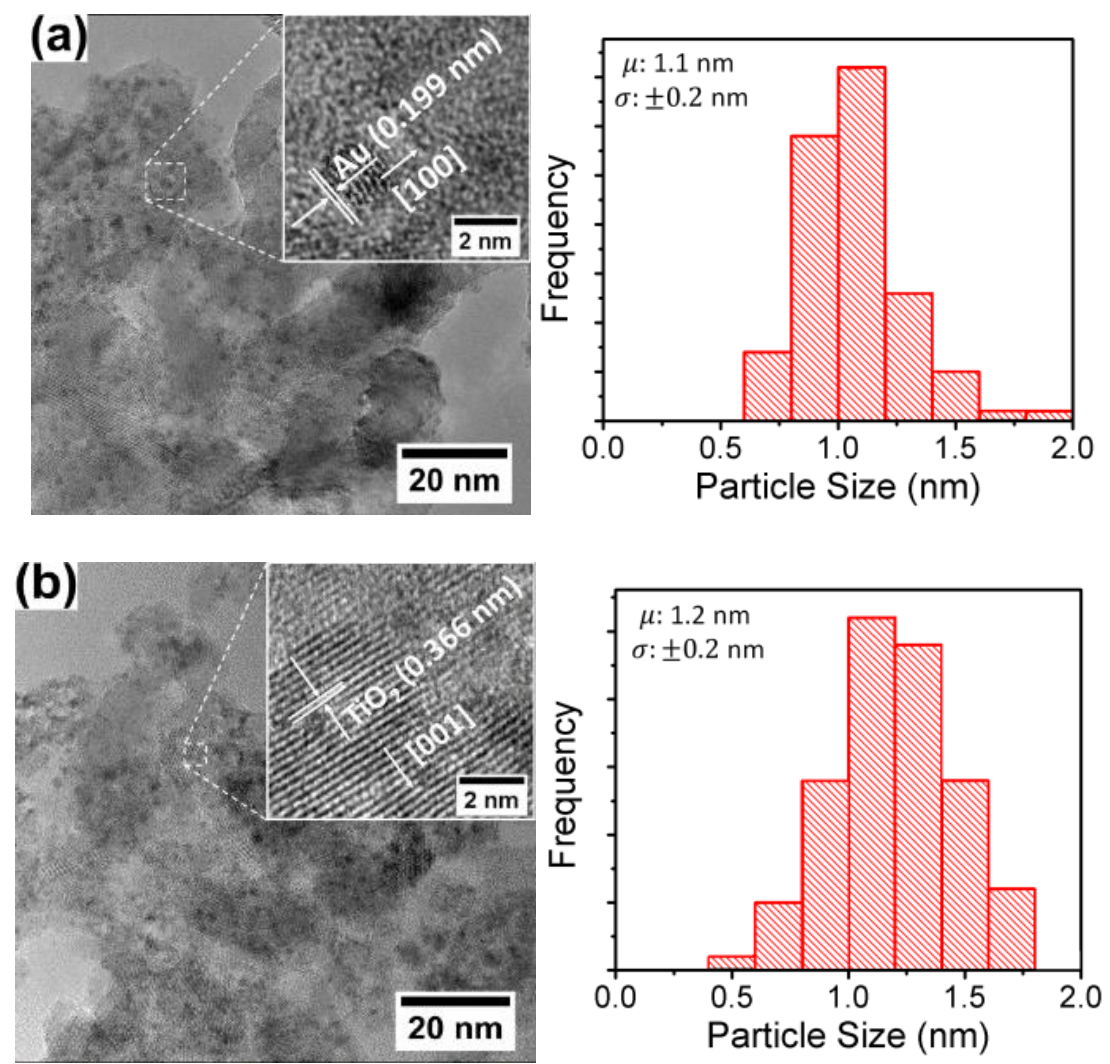

Figure 4.10. TEMs of nanocomposites with their respective Au particle size distribution with an absorbed dose of $7260 \mathrm{~Gy}$ for a) $\mathrm{Au} @ \mathrm{TiO}_{2}{ }^{(21.6 n m)-u r e a}$ and b) $\mathrm{Au} @ \mathrm{TiO}_{2}{ }^{(6.5 n m)-u r e a}$. 


\subsubsection{Surface chemistry of $\mathrm{Au} @ \mathrm{TiO}_{2}$ nanocomposites.}

Analysis of the chemical states and binding energies of the fabricated nanocomposite using $\mathrm{NaOH}$ was done using XPS and results are shown in Figure 4.11. Analysis of the high-resolution Au4f spectrum was fitted for $\mathrm{Au} 4 \mathrm{f}^{7 / 2}$ and $\mathrm{Au} 4 \mathrm{f}^{5 / 2}$ states (Figure 4.11a). The peaks corresponding to $\mathrm{Au}$ $4 \mathrm{f}^{7 / 2}$ were found at $84.04 \mathrm{eV}$ and $85.0 \mathrm{eV}$, and in the case of $\mathrm{Au}, 4 \mathrm{f}^{5 / 2}$ were found at $87.72 \mathrm{eV}$ and $88.70 \mathrm{eV}$. The peaks at $\mathrm{BEs} \approx 84 \mathrm{eV}$ and $\approx 87.7 \mathrm{eV}$ are assigned to $\mathrm{Au}^{(0)}$. In addition, the peaks at binding energies of $\approx 85.0 \mathrm{eV}$ and $\approx 87.7 \mathrm{eV}$ are associated with a small fraction of $\mathrm{Au}^{(\mathrm{I})}$ that was not reduced by the X-rays during synthesis [144]. The fraction corresponding to $\mathrm{Au}^{(\mathrm{I})}$ species is a result of an incomplete reduction of $\mathrm{Au}^{(\mathrm{III})}$ species initially present in the solution Nevertheless, the ratio between the intensities of the peaks of $\mathrm{Au}^{\left(\mathrm{I} / \mathrm{Au}^{(0)}\right.}$ is 0.20 , hence 0.80 of the Au precursor was reduced via X-ray irradiation. Moreover, Figure 4.11b shows the Au-O peak at $530.2 \mathrm{eV}$ which suggests that, during deposition, $\mathrm{Au}$ species bind with $\mathrm{OH}$ groups present on the $\mathrm{TiO}_{2}$ surface [46].
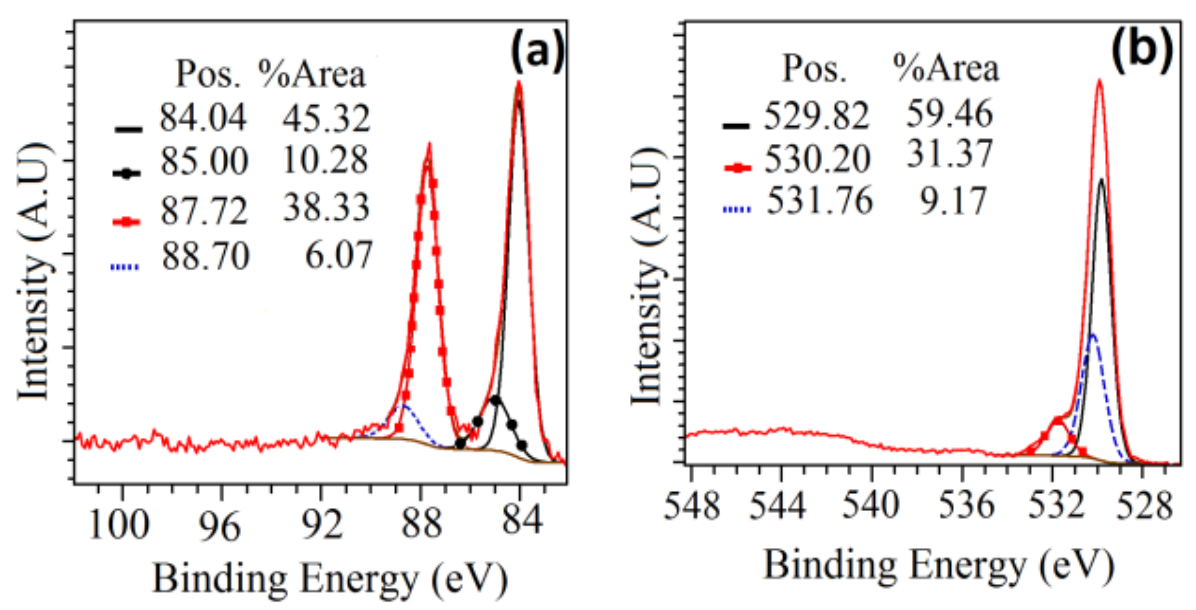

Figure 4.11. HR-XPS spectra of $\mathrm{Au} @ \mathrm{TiO}_{2}{ }^{(21.6 n m)-N a O H}$ nanocomposites using an absorbed dose of 7260 Gy a) Au4f and b) O1s. 
The results presented in this section have been adapted from a manuscript entitled: “ $\mathrm{Au} @ \mathrm{TiO}_{2}$ nanocomposites synthesized by X-ray radiolysis as potential radiosensitizers”. Published in applied surface science.

\subsection{Conclusions}

In this chapter, the development of an innovative synthesis route of $\mathrm{Au} @ \mathrm{TiO}_{2}$ nanocomposites was investigated in detail. Au nanoparticles with narrow particle size distribution were produced on titania supports using clean and simple solvents such as isopropanol and water by means of X-ray irradiation. Control over particle size and particle size distributions was achieved by tuning the absorbed dose and choice of ligand. X-ray radiolytic synthesis is a promising method for increasing the amount of catalytically active sites through enhancement of the nanocomposites features such as nanoparticle loading and nanoparticle-support interactions and monodispersity. The results showed that an increase in the absorbed dose leads to homogenously dispersed nanoparticles with a mean particle size of $\sim 1 \mathrm{~nm}$. This is due to an increase in the production of reactive species such as $\mathrm{e}^{-}$aq that act upon the metal ion in solution producing homogenously distributed seeds. Moreover, the ligands explored in this work, $\mathrm{NaOH}$ and urea showed similar particle size and loading when high absorbed doses (i.e 720 and 7260 Gy) were used. On the other hand, the mean particle size of the support did not affect Au mean particle size and loading onto $\mathrm{TiO}_{2}$. Crystalline structure and surface chemistry characterization of Au nanoparticles were carried out using XRD and XPS respectively. Results showed crystalline Au nanoparticles with a fcc structure, and XPS results suggested that Au binds to the surface of the $\mathrm{TiO}_{2}$ with the $\mathrm{OH}$ groups present on the titania surface produced by the ligands. In this chapter, X-ray radiolysis was positioned as a clean alternative for nanocomposites fabrication. Nowadays, several methods are available to fabricate $\mathrm{Au} @ \mathrm{TiO}_{2}$ nanocomposites, but most are based on wet-chemical methods 
that use chemical reducing agents and require extensive washing or high temperature processing (calcination). In contrast, X-ray radiolysis methodology is compelling by offering a simpler alternative to traditional nanocomposite fabrication routes. This alternative method to produce $\mathrm{Au} @ \mathrm{TiO}_{2}$ nanocomposites is justified by their applications in nanomedicine and catalysis. 


\section{Chapter 5: Comparison of X-ray Radiolytic synthesis with traditional Deposition- precipitation method}

\subsection{Introduction.}

$\mathrm{Au} @ \mathrm{TiO}_{2}$ nanocomposites have been traditionally synthesized through wet chemical routes such as deposition-precipitation. Briefly, the deposition-precipitation approach relies on the precipitation of $\mathrm{Au}$ precursor onto the support in the presence of a ligand ( $\mathrm{NaOH}$ or urea), followed by a calcination process at a temperature of $300{ }^{\circ} \mathrm{C}$ for several hours (3-4 hours) [46]. Although wet chemical methods are commonly used to produce nanocomposites, there are some disadvantages associated with these methods such as broad particle size distribution of the supported nanoparticle, precipitation of carbonaceous species during calcination processes and the need of chemical reducing agents that often result in extensive cleaning processes of the resulting nanocomposite [39]. Nonetheless, there are clean manufacturing techniques capable of producing nanocomposites in the absence of long heat treatments such as radiolytic synthesis. This technique was investigated, as reported in the previous chapter, to successfully produce nanocomposites in the absence of high temperature and pressure. Furthermore, in this synthesis method, the reducing agents are created during radiolysis of water, eliminating the need for toxic chemical reducing agents.

The aim of this chapter is to provide a direct comparison of the nanocomposites obtained by deposition-precipitation and those obtained by X-ray radiolytic synthesis. Herein, we report the synthesis of $\mathrm{Au} @ \mathrm{TiO}_{2}$ nanocomposites, using the deposition-precipitation method and $\mathrm{NaOH}$ as a ligand. Moreover, Au nanoparticles were deposited onto $\mathrm{TiO}_{2}$ with a different particle size $(6.5 \mathrm{~nm}$ and $21.6 \mathrm{~nm}$ ). Parameters such as Au loading, particle size, and particle size distribution were evaluated in the nanocomposites produced by traditional deposition-precipitation and compared with $\mathrm{Au} @ \mathrm{TiO}_{2}$ nanocomposites prepared with X-ray radiolytic synthesis. 


\subsection{Experimental Procedure.}

\subsubsection{Deposition-precipitation of $\mathrm{Au}_{0} \mathrm{TiO}_{2}$ Nanocomposites.}

The deposition-precipitation of $\mathrm{Au} @ \mathrm{TiO}_{2}$ was adapted from the literature [29]. First, a $2 \mathrm{Mm}$ metal precursor solution of $\mathrm{HAuCl}_{4}$ was prepared and the $\mathrm{pH}$ was adjusted to 8 with a $\mathrm{NaOH}$ solution (1 M). Subsequently, $4 \mathrm{mg}$ of $\mathrm{TiO}_{2}$ per $\mathrm{ml}$ of stock solution, with a particle size of either 6.5 or 21.6 $\mathrm{nm}$, was added and sonicated in order to obtain a homogeneous dispersion. After sonication, the $\mathrm{pH}$ was adjusted to a value of 8 . Then, the solution was heated at $80{ }^{\circ} \mathrm{C}$ for 1 hour in order to enable the electrostatic interaction between Au ions and the support. After the heating process, the solution was washed thoroughly. For this purpose, the samples were centrifuged, the supernatant was removed, and fresh DI water was added to the samples to compensate for that removed. The solution was then collected and calcined for $4 \mathrm{~h}$ at $300{ }^{\circ} \mathrm{C}$. After the calcination process, the samples were ground to a fine powder and stored in the dark. The overall experimental procedure is depicted in Figure 5.1.

1. Mixing of the aqueous Solutions

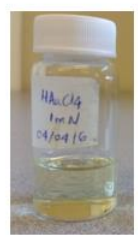

$\mathrm{HAuCl}_{4}$

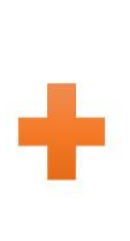

3. Calcination fo 4 hours and $300^{\circ} \mathrm{C}$

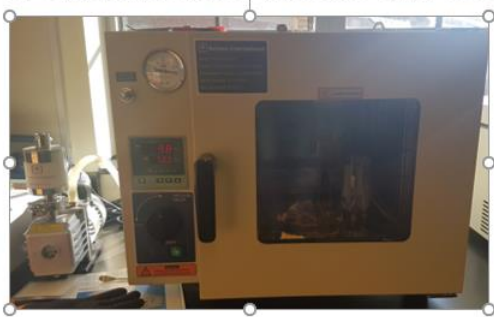

2. Heating of the solution for 1 hour at $80^{\circ} \mathrm{C}$
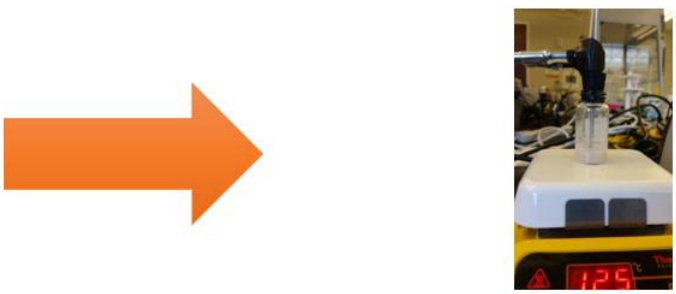

4. Fine powder of Au@TiO 2 NCs
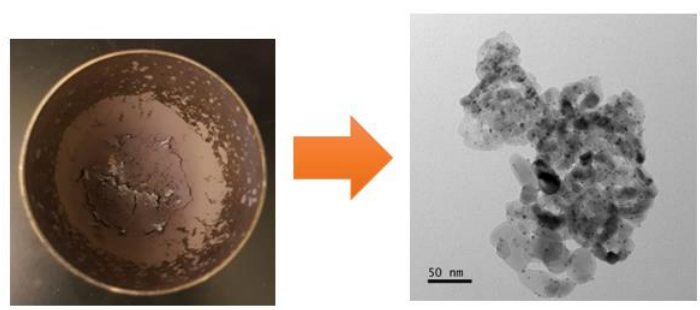

Figure 5.1. Experimental Procedure of $\mathrm{Au} @ \mathrm{TiO}_{2}$ nanocomposites fabricated by the Depositionprecipitation method. 


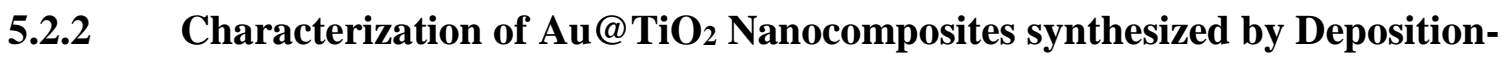 precipitation and $X$-ray radiolytic syntheses.}

The crystalline structure analysis of $\mathrm{Au} @ \mathrm{TiO}_{2}$ fabricated by X-ray radiolysis and the depositionprecipitation method was investigated using a PAnalytical X'Pert Pro X-ray diffractometer with a $\mathrm{Cu}$ target at a current of $40 \mathrm{~mA}$ and an operating voltage of $45 \mathrm{kV}$. For this purpose, the nanopowders were placed onto a low background silicon wafer and loaded onto a spinning sample stage with an angular velocity of $120 \mathrm{rpm}$. The diffraction patterns were analyzed with the software X'pert Highscore. The particle size of $\mathrm{TiO}_{2}$ and $\mathrm{Au}$ supported $\mathrm{TiO}_{2}$ synthesized by both methods was evaluated using TEM using a Zeiss Libra 120 plus operating at a voltage of $120 \mathrm{kV}$. TEM samples were prepared by dispersing the nanopowders in acetone using an ultrasonic probe. A $10 \mu 1$ drop of these solutions was deposited onto a formvar/carbon copper grid. Au Particle size was measured using the software Image J 1.5i. Finally, evaluation of the experimental Au loading onto $\mathrm{TiO}_{2}$ was analyzed by using energy dispersive spectroscopy (EDX) in a Phenom microscope ProX SEM at an operating voltage of $15 \mathrm{kV}$. For this purpose, $\mathrm{Au} @ \mathrm{TiO}_{2}$ nanopowders were dispersed in acetone using a vortex mixer. Afterward, a drop of the solution was deposited onto a low background silicon wafer.

\subsection{Results and discussion.}

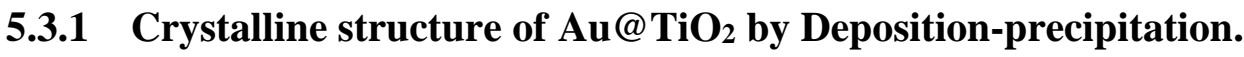

The XRD patterns of $\mathrm{Au} @ \mathrm{TiO}_{2}$ nanocomposites synthesized either with X-ray radiolysis (a-b) or deposition-precipitation method (c-d) using $\mathrm{TiO}_{2}$ with different particle size are shown in Figure 5.2. The patterns of $\mathrm{Au} @ \mathrm{TiO}_{2}$ synthesized with the deposition-precipitation method, showed the characteristic peaks of Anatase $\mathrm{TiO}_{2}$ (pdf: 01-075-2552) and Au with an fcc crystalline structure (pdf: 01-071-4614). Interestingly, the Au peaks in the samples fabricated with deposition- 
precipitation have low relative intensity when compared with the nanocomposites fabricated with radiolysis. This is due to the inferior loading of $\mathrm{Au}$ onto $\mathrm{TiO}_{2}$ when synthesized using calcination.

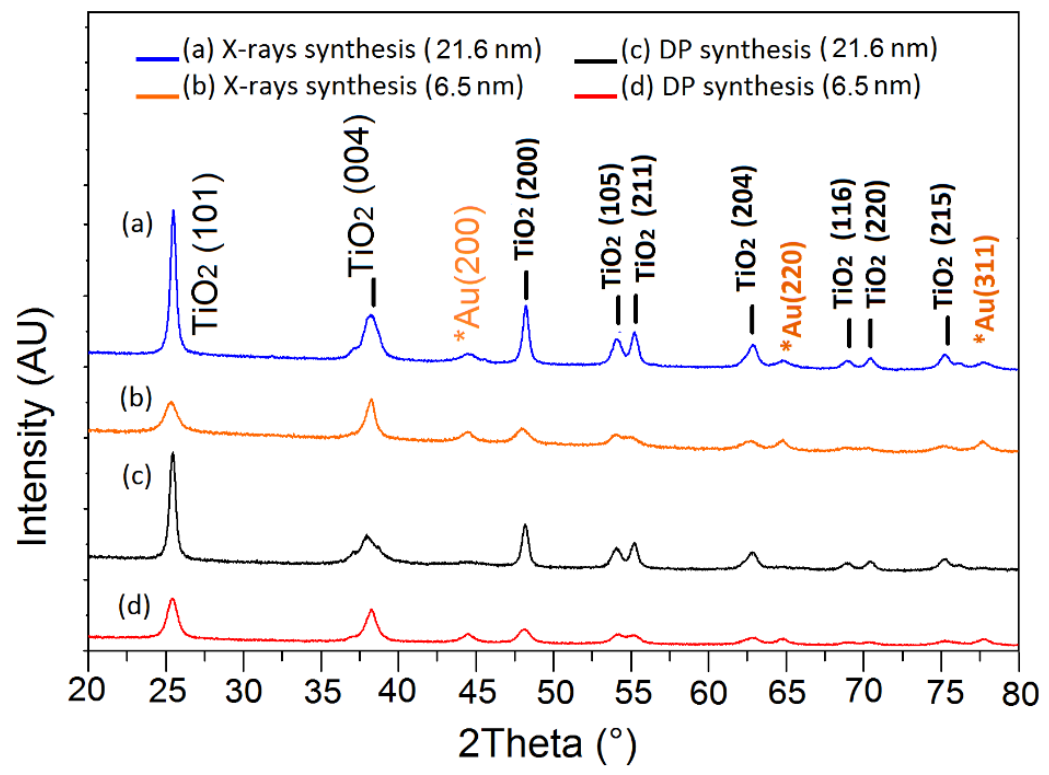

Figure 5.2. $\mathrm{XRD}$ of $\mathrm{Au} @ \mathrm{TiO}_{2}$ nanocomposites using radiolytic synthesis and Depositionprecipitation method.

\subsubsection{Morphology of $\mathrm{Au}_{0} \mathrm{TiO}_{2}$ synthesized by Deposition-precipitation.}

The morphology of $\mathrm{Au} @ \mathrm{TiO}_{2}$ synthesized by the deposition-precipitation method is shown in Figure 5.3. TEM results of $\mathrm{Au} @ \mathrm{TiO}_{2}$ using the traditional deposition-precipitation method using $21.6 \mathrm{~nm} \mathrm{TiO}_{2}$ are shown in figureFigure 5.3a and $\mathrm{Au} @ \mathrm{TiO}_{2}$ supported on $6.5 \mathrm{~nm}$ titania are observed in figure Figure 5.3b. During calcination, the precipitates of $\mathrm{Au}(\mathrm{OH})_{3}$ created during the heating process transform into Au metal seeds when the calcination temperature is above $200{ }^{\circ} \mathrm{C}$ [145]. These seeds coalesce, ultimately forming Au nanoparticles. Analysis of the Au particle size supported on $21.6 \mathrm{~nm}$ and $6.5 \mathrm{~nm}$ titania showed values of $4.1 \pm 0.8 \mathrm{~nm}$ and $6.7 \pm 7.0$ respectively. The large mean particle size and standard deviation of Au supported on $6.5 \mathrm{~nm} \mathrm{TiO}_{2}$ is due to the isoelectric point of the supports. The deposition-precipitation method using $\mathrm{NaOH}$ has been shown to be ineffective on some metal oxides because Au hydroxides cannot be deposited on materials 
with an isoelectric point (IEP) below 5 [145]. Evaluation of the IEP of the $6.5 \mathrm{~nm}$ and $21.6 \mathrm{~nm}$ $\mathrm{TiO}_{2}$ supports was made using zeta potential and IEP values of 2.1 and 5.5 were obtained respectively. Because the IEP of the $6.5 \mathrm{~nm}$ support is below $5, \mathrm{NaOH}$ is not a suitable ligand while using calcination processes for the synthesis of $\mathrm{Au} @ \mathrm{TiO}_{2}$ nanocomposites.
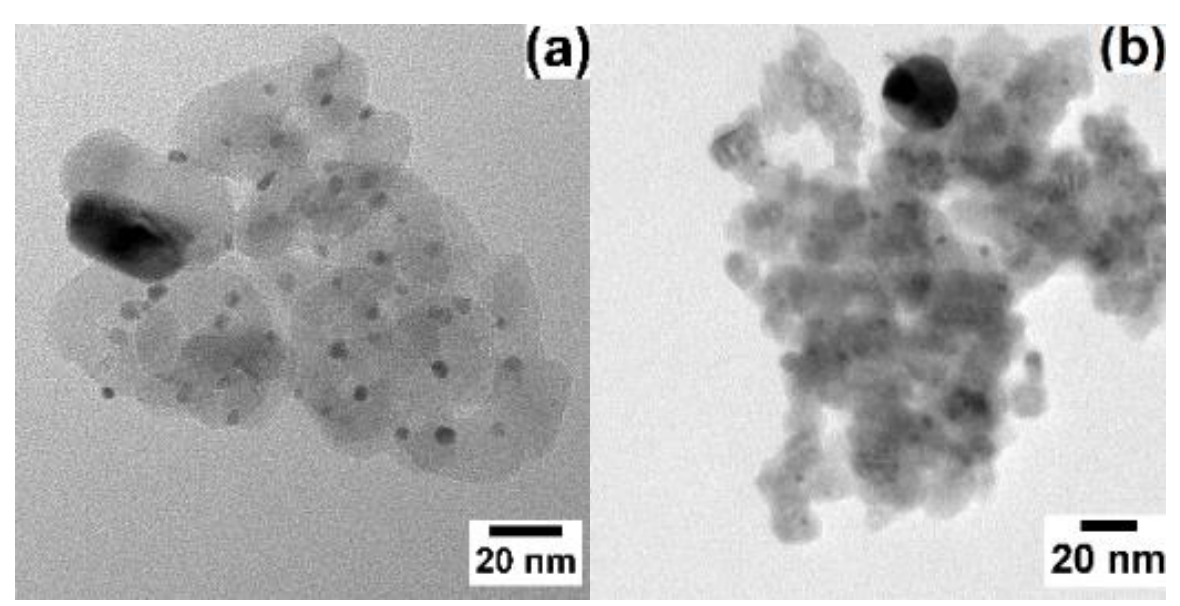

Figure 5.3. Au@ $\mathrm{TiO}_{2}$ nanocomposites fabricated with the Deposition-precipitation method onto a) $21.6 \mathrm{~nm}$ supports b) $6.5 \mathrm{~nm}$ supports.

\subsubsection{Loading comparison of $\mathrm{Au}_{\mathrm{u}} \mathrm{TiO}_{2}$ synthesized by $\mathrm{X}$-ray radiolysis and the Deposition-precipitation method.}

EDX analysis showed a superior loading when Au was supported on $\mathrm{TiO}_{2}$ using X-ray radiolytic synthesis, as shown in Table 5.1. The loading was evaluated by comparing Au deposition onto $\mathrm{TiO}_{2}$ with a mean particle size of $21.6 \mathrm{~nm}$. The loading found on $\mathrm{Au} @ \mathrm{TiO}_{2}$ fabricated with X-ray radiolytic synthesis was $8.9 \%$, whereas a loading of $6.6 \%$ was found when the synthesis method was deposition-precipitation. One of the ways to improve Au deposition yield in the depositionprecipitation method is to increase Au concentration in solution during synthesis, however, the average particle size grows larger and the particle size distribution becomes broader [29]. X-ray radiolytic synthesis showed a high Au deposition yield, without the need of increasing the concentration of Au precursor in solution, this shows that X-ray radiolytic synthesis is an effective 
route to deposit $\mathrm{Au}$, as it optimizes the use of the precursor, and led to a uniform distribution of particles with a small size.

Table 5.1. Loading of $\mathrm{Au}$ supported $\mathrm{TiO}_{2}$ by $\mathrm{X}$-ray radiolytic synthesis and the Depositionprecipitation method.

\begin{tabular}{|c|c|}
\hline Sample & Loading \\
\hline $\mathrm{Au} @ \mathrm{TiO}_{2}(21.6 \mathrm{~nm})$ by X-rays & $8.9 \%$ \\
\hline $\mathrm{Au} @ \mathrm{TiO}_{2}(21.6 \mathrm{~nm})$ by Deposition-precipitation method & $6.6 \%$ \\
\hline
\end{tabular}

\subsubsection{Heating process relevance in $\mathrm{X}$-ray radiolytic synthesis.}

The heating process with $\mathrm{NaOH}$ ensures that the Au nanoparticles will nucleate and grow onto the $\mathrm{TiO}_{2}$ during the subsequent irradiation process. During the heating process, the depositionprecipitation method involves the deposition of $\mathrm{Au}^{(\mathrm{III})}$ and $\mathrm{Au}^{(\mathrm{I})}$ onto the titania surface. Figure 5.4a shows $\mathrm{Au} @ \mathrm{TiO}_{2}$ nanocomposites synthesis with X-rays in the absence of the heating process, whereas Figure 5.4b shows the UV-Vis pattern of $\mathrm{Au} @ \mathrm{TiO}_{2}$ nanocomposites synthesized with $\mathrm{X}$ rays in the presence and absence of heating. The mean particle size of Au in the absence of heating was $88.5 \pm 23.1 \mathrm{~nm}, \mathrm{UV}-\mathrm{Vis}$ shows a peak at $\sim 545 \mathrm{~nm}$, associated with the surface Plasmon resonance of Au nanoparticles. UV-Vis results showed a broad peak with inferior intensity when $\mathrm{Au} @ \mathrm{TiO}_{2}$ were synthesized using x-rays in the absence of heating, which is also evidence of larger Au nanoparticle size and low loading onto the support. 

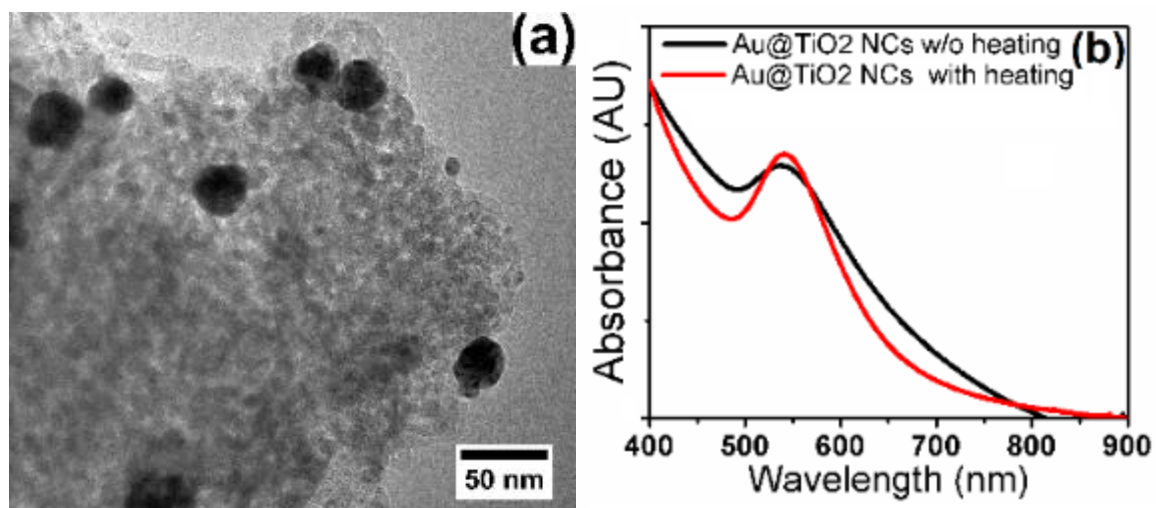

Figure 5.4. a) $\mathrm{TEM}$ of $\mathrm{Au} @ \mathrm{TiO}_{2}$ synthesized in the absence of the heating process. b) UV-Vis of $\mathrm{Au} @ \mathrm{TiO}_{2}$ synthesized in the presence and in the absence of heating.

\subsection{Conclusions.}

$\mathrm{Au} @ \mathrm{TiO}_{2}$ nanocomposites were successfully produced through X-ray radiolysis and depositionprecipitation method. In this chapter, X-ray radiolytic synthesis was compared with the depositionprecipitation method, a more traditional approach where Au nanoparticles are produced using high temperatures. In contrast, X-ray radiolytic synthesis is a methodology carried out at ambient temperature and pressure in the absence of toxic chemical agents. Results showed that $\mathrm{Au}$ nanoparticle loading onto titania was superior by $\sim 35 \%$ when $\mathrm{X}$-ray radiolysis was used as a synthesis method. Furthermore, Au nanoparticles synthesized by X-rays radiolysis were smaller by a factor of $\sim 3.2$ when compared with the Au mean particle size obtained by the depositionprecipitation method. Control on the $\mathrm{Au}$ nanoparticle mean particle size is important for applications such as catalysis and radiosensitization since smaller particles have evidenced a higher catalytic performance. X-ray radiolytic synthesis offers the advantage of producing pure $\mathrm{Au} @ \mathrm{TiO}_{2}$ nanocomposites that can be used in several medical and catalytic applications. 


\section{Chapter 6: Au@ $\mathrm{TiO}_{2}$ as Potential radiosensitizers}

\subsection{Introduction.}

The interaction of ionizing radiation with nanomaterials, holds promising applications in various nuclear and medical fields, particularly in radiation therapy. In this regard, radiosensitizers are of great importance due to their ability to increase cell sensitivity to the effects of radiation. In this chapter, the radiation enhancement response of $\mathrm{Au} @ \mathrm{TiO}_{2}$ nanocomposites was evidenced by monitoring $\mathrm{MB}$ decomposition in the presence of the nanomaterial. The response of the nanomaterial was investigated as a function of parameters such as $\mathrm{Au}$ loading, $\mathrm{TiO}_{2}$ particle size, ligand used for synthesis, nanocomposite concentration, irradiation voltage, and dose rate. $\mathrm{Au} @ \mathrm{TiO}_{2}$ nanocomposites were fabricated by the X-ray synthesis approach described in previous

chapters. Results showed that the addition of $\mathrm{Au} @ \mathrm{TiO}_{2}$ to $\mathrm{MB}$ accelerates the dye reaction rate by increasing the number of reactive oxygen species in solution. Furthermore, the highest values were achieved when using $\mathrm{TiO}_{2}$ with $6.5 \mathrm{~nm}$ in particle size, high $\mathrm{Au}$ loading, and $\mathrm{NaOH}$ as the ligand. This combination of parameters led to an increase in the number of active sites in the nanomaterial, facilitating $\mathrm{MB}$ decomposition. Moreover, $\mathrm{Au} @ \mathrm{TiO}_{2}$ radiosensitization was also tested in a biological environment with M. aeruginosa cells. Where the presence of the nanocomposites during irradiation increased significantly cell damage, diminishing the overall cell sample population. In this chapter the great promise of $\mathrm{Au} @ \mathrm{TiO}_{2}$ and other oxides as radiation enhancers is explained, a feature that is of relevance to a number of medical, biological and environmental applications. 


\subsection{Experimental Procedure.}

\subsubsection{Materials and Reagents.}

Methylene Blue, sodium chloride $(\mathrm{NaCl})$, and ammonium iron(II) sulfate hexahydrate $\left(\left(\mathrm{NH}_{4}\right)_{2} \mathrm{Fe}\left(\mathrm{SO}_{4}\right)_{2} \cdot 6 \mathrm{H}_{2} \mathrm{O}\right)$ were purchased from Acros Organics. M. aeruginosa was purchased from Carolina Biological. Finally, Ultra-pure (18 M $)$ ) deionized (DI) water was obtained from a MilliQ® water purification system.

\subsubsection{Radiosensitization Analysis of $\mathrm{Au} @ \mathrm{TiO}_{2}$ Nanocomposites using Methylene Blue.}

Radiosensitization evaluation of $\mathrm{Au} @ \mathrm{TiO}_{2}$ nanocomposites was made by using a $\mathrm{MB}$ solution as a chemical probe, the solution was then irradiated with X-rays in the presence of the nanomaterial. For this purpose, powders of $\mathrm{Au} @ \mathrm{TiO}_{2}$ nanocomposites synthesized using X-ray radiolysis were added in concentrations of 0.2 and $1 \mathrm{mg} / \mathrm{ml}$ to a $50 \mu \mathrm{M}$ aqueous solution of $\mathrm{MB}$ and placed in 1.5 $\mathrm{ml}$ polypropylene vial. Prior to irradiation, the aqueous solutions containing the nanomaterial were stirred in the dark to ensure the adsorption equilibrium of the MB on the surface of the nanomaterial. In order to evaluate the effect that the energy of the X-rays has on $\mathrm{Au} @ \mathrm{TiO}_{2}$ radiosensitization, the solution was irradiated in a Precision X-ray 225XL using a fixed dose rate of $\sim 35 \mathrm{~Gy} / \mathrm{min}$ and two different output voltages, $50 \mathrm{kV}$ and $225 \mathrm{kV}$. The dose rate was measured using an ionization chamber and further verified with a Fricke dosimeter solution. The dose rate influence on $\mathrm{Au} @ \mathrm{TiO}_{2}$ radiosensitization was tested using also a lower dose rate of $3 \mathrm{~Gy} / \mathrm{min}$ at an operating voltage of $225 \mathrm{kV}$. The MB degradation was analyzed by following the decrease of its characteristic absorbance peak at $664 \mathrm{~nm}$ upon interaction when radiation. MB degradation analysis was carried out using a Genesis 10S UV-Vis spectrophotometer. In order to quantitatively evaluate $\mathrm{MB}$ degradation, the reaction rate constants of $\mathrm{MB}$ were calculated using the absorbance values. A schematic representation of the experimental procedure is shown in Figure 6.1. 


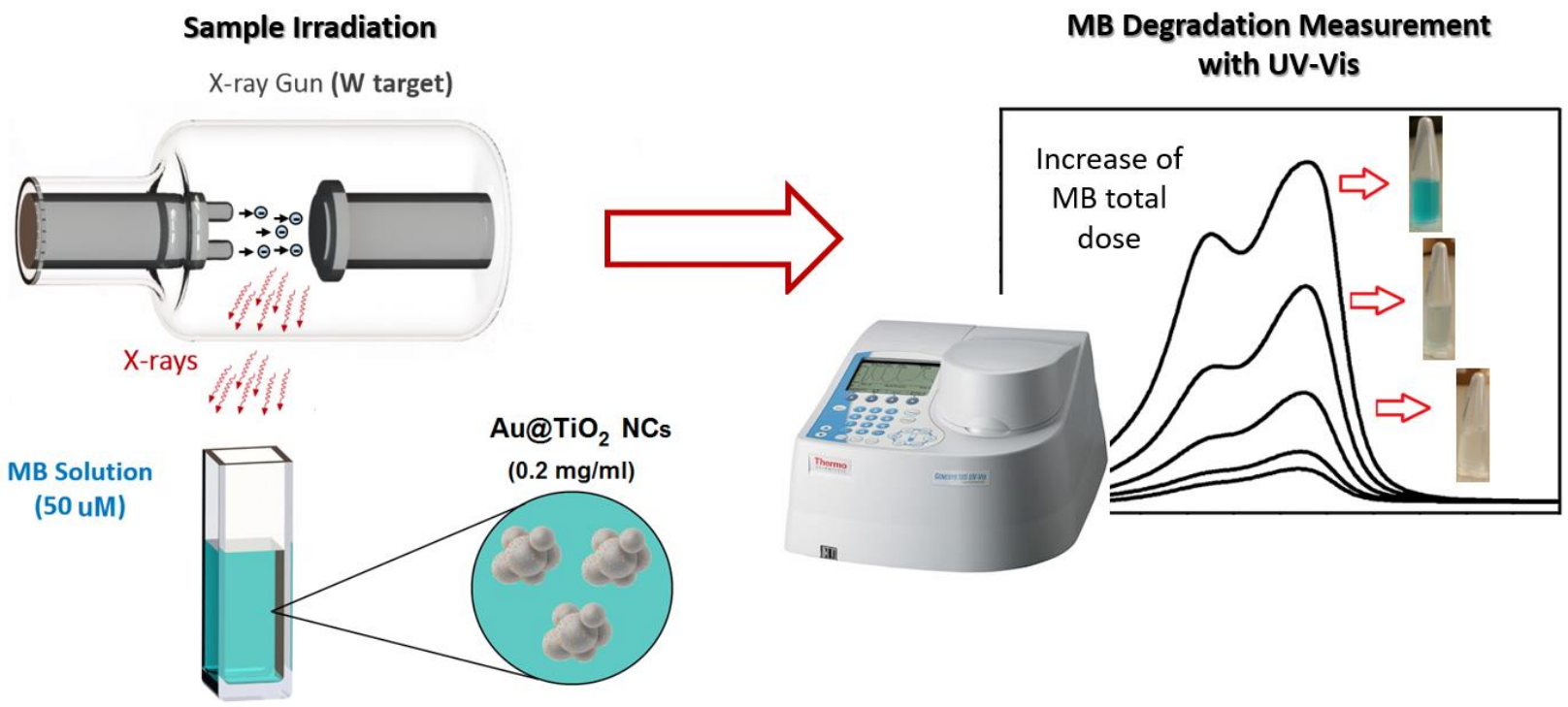

Figure 6.1. Schematic representation of $\mathrm{Au} @ \mathrm{TiO}_{2}$ irradiation on $\mathrm{MB}$ solution and their subsequent degradation measurement.

\subsubsection{Radiosentization of $\mathrm{Au} @ \mathrm{TiO}_{2}$ on Microcystis aeruginosa Cells.}

The radiosensitization evaluation of the nanomaterial on $\mathrm{M}$. aeruginosa was evaluated by adding either $\mathrm{TiO}_{2}{ }^{(21.6 \mathrm{~nm})-\mathrm{NaOH}-10 \%}$ and $\mathrm{Au} @ \mathrm{TiO}_{2}{ }^{(21.6 \mathrm{~nm})-\mathrm{NaOH}-10 \%}$ in a concentration of $0.2 \mathrm{mg} / \mathrm{ml}$ to an aqueous solution containing the cells. Subsequently, the solution was placed in a polypropylene vial and irradiated in a Precision X-ray 225XL at a voltage of $225 \mathrm{kV}$ and a dose rate of 127 $\mathrm{Gy} / \mathrm{min}$, until an absorbed dose of $6 \mathrm{kGy}$ was attained. In order to evaluate cell damage caused by the combined action of irradiation and the radiosensitizers, the chlorophyll concentration was measured on the UV-Vis for 5 consecutive days after irradiation at a wavelength of $679 \mathrm{~nm}$. Furthermore, the interaction of $\mathrm{TiO}_{2}$ and $\mathrm{Au} @ \mathrm{TiO}_{2}$ was evaluated using SEM before and immediately after irradiation. For this purpose, an aliquot containing the cells in the presence of the nanomaterial was placed in a silicon wafer and allowed to dry. Then, the extent of the cell 
physical damage was qualitatively evaluated. The experimental set up of the M. aeruginosa irradiation is shown in Figure 6.2.

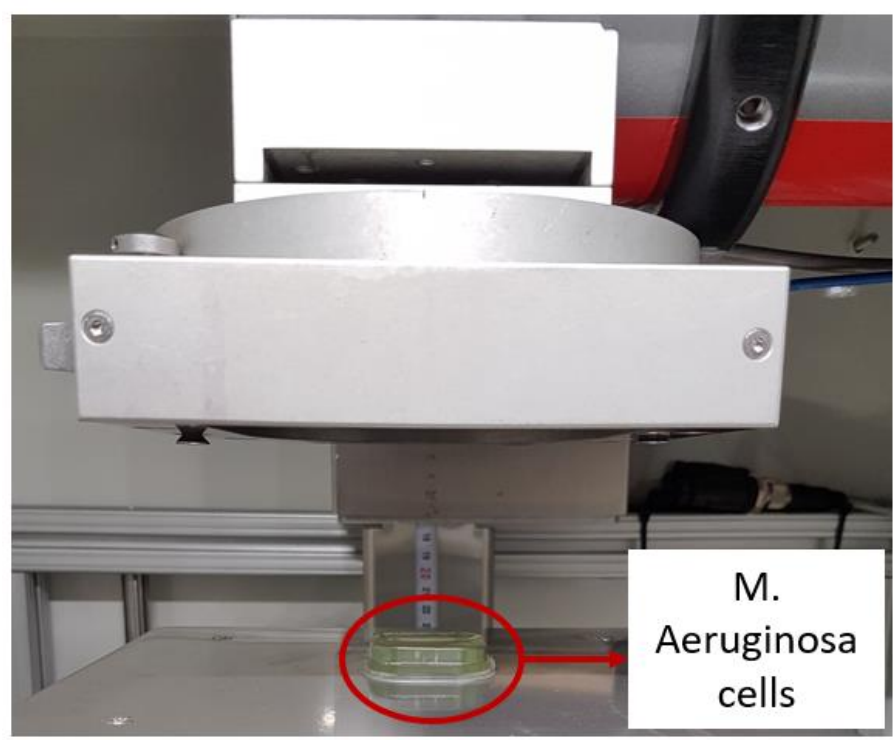

Figure 6.2. Irradiation set up of $\mathrm{M}$. aeruginosa cells containing $\mathrm{TiO}_{2}$ and $\mathrm{Au} @ \mathrm{TiO}_{2}$ nanoparticles.

\subsection{Results and Discussion.}

\subsubsection{Ligand influence on $\mathrm{Au} @ \mathrm{TiO} 2$ radiosensitization.}

The degradation rate of $\mathrm{MB}$ in the presence of $\mathrm{Au} @ \mathrm{TiO}_{2}$ fabricated with either $\mathrm{NaOH}$ or urea is shown in Figure 6.3, where the reaction rate of MB degradation under X-rays in the presence of Au supported on $\mathrm{TiO}_{2}$ with a mean particle size of $6.5 \mathrm{~nm}$ was evaluated. These results evidence that a higher $\mathrm{MB}$ reaction rate is obtained when $\mathrm{NaOH}$ is used as a ligand. A reaction rate constant enhancement of $9.7 \%$ and $65.2 \%$ was observed for $\mathrm{Au} @ \mathrm{TiO}_{2}{ }^{(6.5 \mathrm{~nm})-\mathrm{urea}-10 \%}$ and $\mathrm{Au} @ \mathrm{TiO}_{2}{ }^{(6.5 \mathrm{~nm})-}$ $\mathrm{NaOH}-10 \%$ respectively. During synthesis, ligands are added to the aqueous solutions prior to irradiation in order to ensure that the Au nanoparticles are tethered onto the $\mathrm{TiO}_{2}$ surface. The ligands are activated through a heating process that allows for the binding and the deposition of $\mathrm{Au}^{3+}$ and $\mathrm{Au}^{1+}$ ions onto titania $[39,46]$. The metal precursor $\mathrm{HAuCl}_{3}$ reacts with $\mathrm{NaOH}$, in a 
process that results in a deposition of chloro-hydroxo Au species onto the surface of the oxide. Furthermore, the heating process, enables a gradual increment of $\mathrm{OH}$ groups on the surface of the support, evidenced by a $\mathrm{pH}$ increment in solution [45]. The maximum deposition of Au species onto $\mathrm{TiO}_{2}$ is found to be between $\mathrm{pH}$ ranging from 6 to 8 , in a process characterized by the deposition of $\mathrm{Au}(\mathrm{OH})_{3}$ onto the support surface [45]. When $\mathrm{NaOH}$ is used as ligand, the deposition of $\mathrm{Au}$ ions onto the $\mathrm{TiO}_{2}$ surface and the formation of $\mathrm{Au}-\mathrm{OH}$ bonds takes place within one hour of heating [29]. On the other hand, the formation of $\mathrm{Au}-\mathrm{OH}$ bonds in the presence of urea is a fourhour process. When urea is heated above $60{ }^{\circ} \mathrm{C}, \mathrm{OH}^{-}$ions are released into the aqueous medium, gradually increasing the $\mathrm{pH}$ from 2 to 8 , in a process known as hydrolysis of urea. This causes slow precipitation of the Au hydroxides onto the titania support [46]. However, the utilization of long-chain ligands such as urea $\left(\mathrm{NH}_{2} \mathrm{CONH}_{2}\right)$ during synthesis can be counterproductive in some medical and catalytical applications, since they might act as physical and chemical barriers for the nanomaterial to perform [120]. In general, the successful application of nanomaterials after synthesis is dependent on a surface free of solvents and residual by-products that might cause poor catalytic activity. The use of smaller molecules is a more benign approach that can be followed to produce nanoparticles with minimal residues [120]. Radiosensitization assessment of the nanocomposites synthesized with $\mathrm{NaOH}$ and urea showed that, when $\mathrm{Au} @ \mathrm{TiO}_{2}$ were fabricated using small molecules in a lower concentration such as $\mathrm{NaOH}$, leads to a higher radiosensitization. The carbon peak intensity on the $\mathrm{Au} @ \mathrm{TiO}_{2}$ nanocomposites fabricated with urea was found to be $\sim 8.5$ times higher than that in nanocomposites fabricated with $\mathrm{NaOH}$ per $\mathrm{X}$-ray photoelectron spectroscopy (XPS) results (Figure 6.4a). Moreover, composition analysis made by XPS showed a carbon content of 15.9 and 24.6 wt $\%$ for $\mathrm{Au} @ \mathrm{TiO}_{2}{ }^{21.6} \mathrm{~nm}$, fabricated with $\mathrm{NaOH}$ and urea 
respectively. Thus, residual ligand in $\mathrm{Au} @ \mathrm{TiO}_{2}$ nanocomposites synthesized with urea remained in the dried powder of $\mathrm{Au} @ \mathrm{TiO}_{2}$ nanocomposites even after extensive washing.

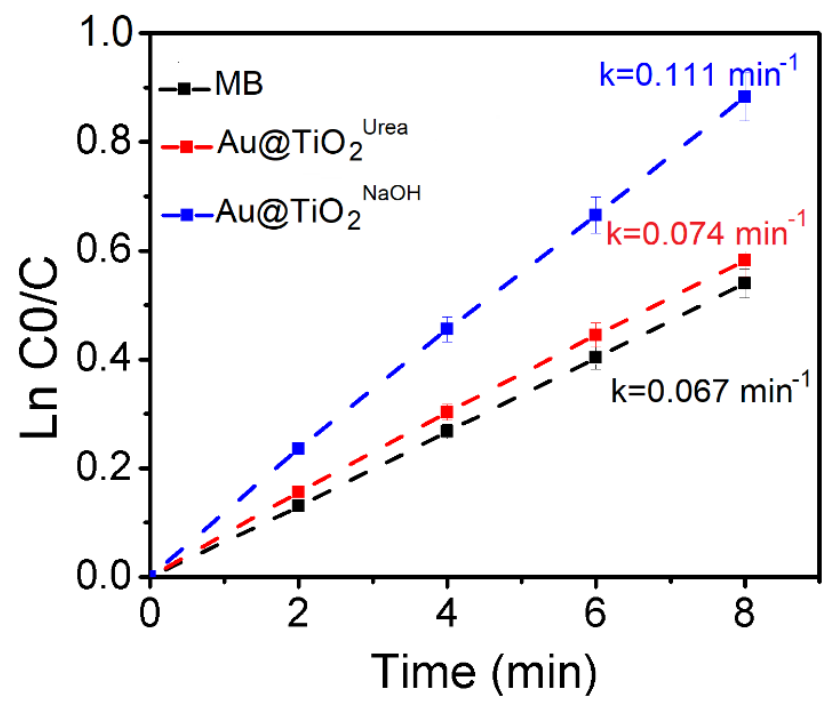

Figure 6.3. Kinetics of MB decrease under X-rays in the presence of $\mathrm{Au} @ \mathrm{TiO}_{2}{ }^{(6.5 \mathrm{~nm})-\mathrm{NaOH}-10 \%}$ and $\mathrm{Au} @ \mathrm{TiO}_{2}{ }^{(6.5 \mathrm{~nm})-U r e a-10 \%}$ at a concentration of $0.2 \mathrm{mg} / \mathrm{ml}$. Irradiation carried out at $50 \mathrm{kV}$ and a dose rate of $35 \mathrm{~Gy} / \mathrm{min}$.
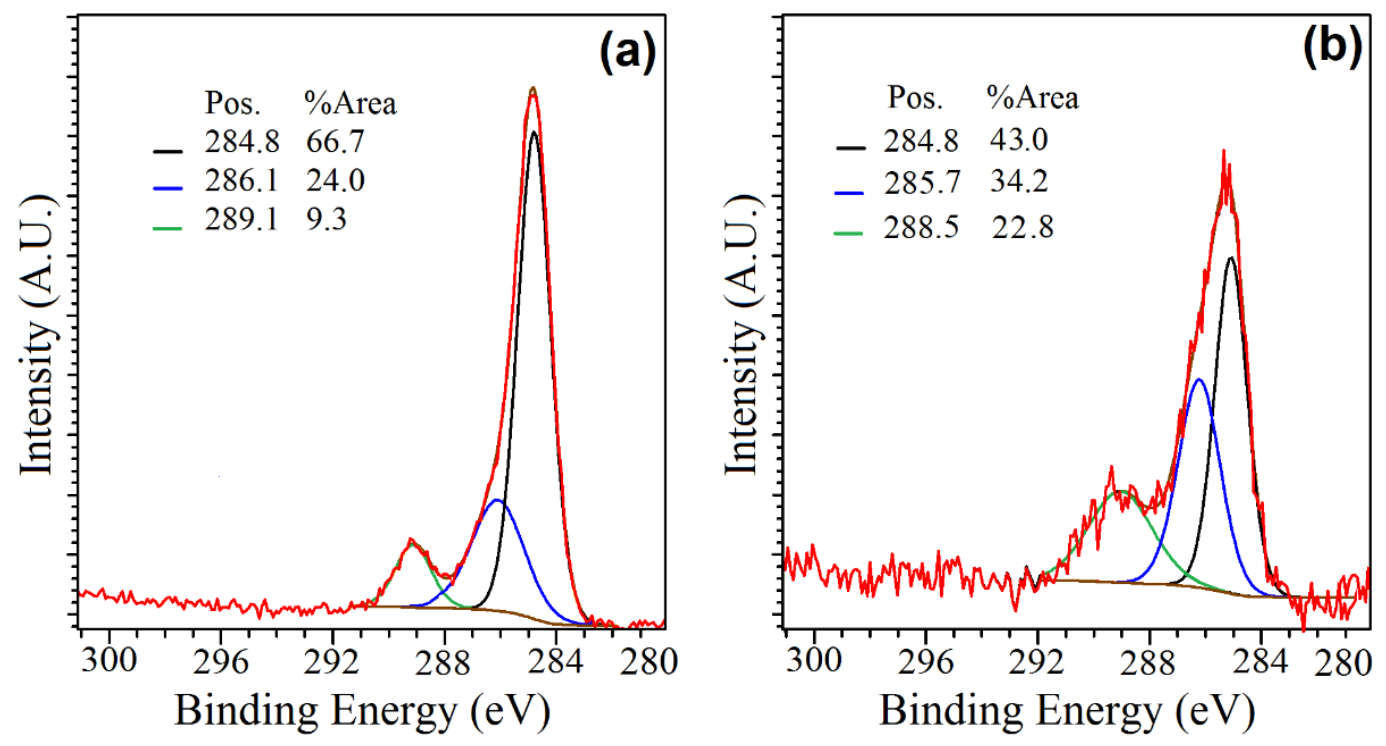

Figure 6.4. $\mathrm{C} 1 \mathrm{~s}$ HR-XPS Analysis of a) $\mathrm{Au} @ \mathrm{TiO}_{2}{ }^{(21.6 \mathrm{~nm})-\mathrm{Urea}-10 \%}$ b) $\mathrm{Au} @ \mathrm{TiO}_{2}{ }^{(21.6 \mathrm{~nm})-\mathrm{NaOH}-10 \%}$ 
The radiosensitization of the bare $\mathrm{TiO}_{2}$ was also measured and Figure 6.5 shows the reaction rate of $\mathrm{MB}$ in the presence of $\mathrm{TiO}^{(6.5 \mathrm{~nm})}$ at different concentrations. Although the reaction rate constants of $\mathrm{MB}$ in the presence of $\mathrm{TiO}_{2}$ concentration of $0.2 \mathrm{mg} / \mathrm{ml} \mathrm{MB}$ was $0.043 \mathrm{~min}^{-1}$, an increase of their concentration to $1 \mathrm{mg} / \mathrm{ml}$ led to a reaction rate constant of $0.073 \mathrm{~min}^{-1}$. Radiosensitization studies have reported a phenomenon known as anti-enhancement, where certain concentrations of nanoparticles in solution seem to scavenge $\mathrm{OH}^{\bullet}$. This scavenging process results in none or low radiation enhancement [117]. The anti-enhancement phenomenon is observed in Figure 6.5 at a $\mathrm{TiO}_{2}$ concentration of $0.2 \mathrm{mg} / \mathrm{ml}[146]$.

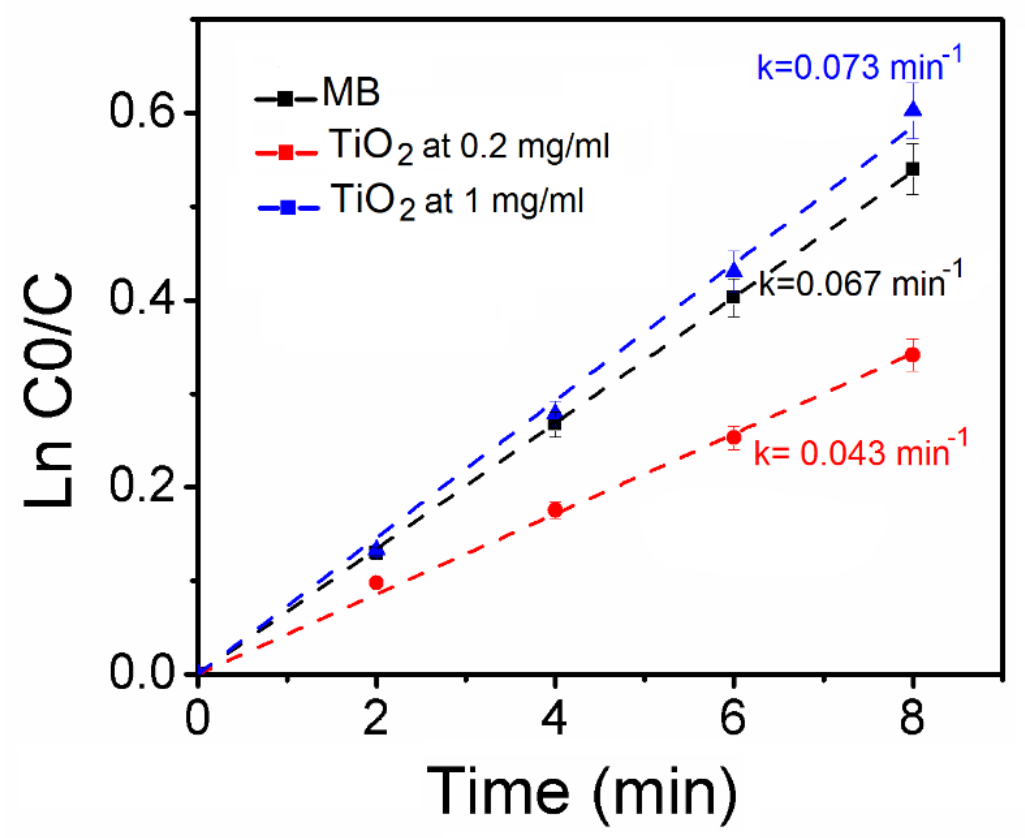

Figure 6.5. Reaction rate of $\mathrm{MB}$ using $\mathrm{X}$-rays in the presence of $\left.\mathrm{TiO}_{2}{ }^{(6.5} \mathrm{nm}\right)$ at different nanomaterial concentration. Irradiation carried out at $50 \mathrm{KV}$ and $35 \mathrm{~Gy} / \mathrm{min}$.

\subsubsection{Radiation enhancement by $\mathrm{Au}_{0} \mathrm{TiO}_{2}$ and $\mathrm{TiO}_{2}$.}

Radiosensitization of bare $\mathrm{TiO}_{2}$ and $\mathrm{Au}$ decorated titania are observed in Figure 6.6, where the MB degradation kinetics was evaluated giving reaction rate constants of $0.067 \mathrm{~min}^{-1}, 0.073 \mathrm{~min}^{-1}$ and $0.105 \mathrm{~min}^{-1}$ for $\mathrm{MB}, \mathrm{MB}$ in the presence of $\mathrm{TiO}_{2}$, and $\mathrm{MB}$ containing $\left.\mathrm{Au} @ \mathrm{TiO}_{2}{ }^{(6.5} \mathrm{nm}\right)-\mathrm{NaOH}-10 \%$ 
respectively. The $\mathrm{MB}$ solutions containing $\mathrm{Au} @ \mathrm{TiO}_{2}$ nanocomposites showed a $43.8 \%$ higher enhancement over that with bare $\mathrm{TiO}_{2}$, evidencing the ability of Au nanoparticles to increase the number of ROS in solution. Overall, the addition of $\mathrm{Au}$ onto titania surface modifies their electronic band structure leading to a reduction in the recombination rate of carriers, thus increasing the radiosensitization effect of the nanocomposites. Furthermore, the interaction of the support with ionizing radiation is known to generate oxygen vacancies in the surface of titania, promoting water dissociation and increasing the amount of $\mathrm{OH}^{\circ}$ on the surrounding medium [147]. Nonetheless, the most important reason behind the significant increase of MB reaction rate in the presence of $\mathrm{Au} @ \mathrm{TiO}_{2}$ nanocomposites is the combination of the electron-hole pairs produced by $\mathrm{TiO}_{2}$ and the photoemissions created upon interaction of $\mathrm{Au}$ with X-rays $[3,9,17]$. The excitation and ionization processes caused by the interaction of the photoemissions with the water molecules leads to the production of free radicals such as $\mathrm{OH}^{\bullet}, \mathrm{O}_{2}^{-\bullet}$ and $\mathrm{H}^{+}$. These species interact with the $\mathrm{MB}$, leading to a semi-reduced state of the dye known as $\mathrm{MB}^{\bullet}$, in a process known to as chemical enhancement $[116,148,149]$. This is associated with the increase in the production of radicals that will cause permanent damage to the dye, resulting in an accelerated reaction rate [17]. From here, the decomposition of $\mathrm{MB}^{\bullet-}$ can take two pathways, the first path is associated with the encountering of another $\mathrm{MB}^{\bullet-}$ molecule to form a $\mathrm{MB}$ and leuco-methylene (LMB) which is a double reduced form of MB. The second path is related to $\mathrm{MB}^{\bullet-}$ and its reaction with $\mathrm{e}^{-} \mathrm{CB}$ and $\mathrm{H}^{+}$, which also leads to LMB. The formation of LMB leads to a decrease in the absorbance at $664 \mathrm{~nm}$ and discoloration of the dye [148]. The $\mathrm{OH}^{\bullet}$ radicals also play an important role in the degradation of $\mathrm{MB}$, since they are interacting with the dye produces $\mathrm{MB}^{2+}$ and $\mathrm{MBOH}^{+}$species that accelerate the discoloration [150]. 
The degradation of MB in the absence of particles follows the same pathway aforementioned, where the interaction of $\mathrm{MB}$ molecules with photons will lead to species such as $\mathrm{MB}^{\bullet-}, \mathrm{LMB}$, $\mathrm{MB}^{2+}$ and $\mathrm{MBOH}^{+}$. After irradiation, a decrease of the absorbance of the peaks at 256 and $664 \mathrm{~nm}$ was observed, these peaks represent LMB and MB respectively. The decrease in absorbance of these peaks is associated with successful degradation of both MB and LMB by X-rays. The addition of $\mathrm{Au} @ \mathrm{TiO}_{2}$ to the solution causes an accelerated dye decomposition as a direct effect of the increase of ROS species in solution produced upon X-rays interaction with the nanomaterial. At the voltage and dose rate conditions tested in Figure 6.6, full discoloration of the dye in the presence of $\mathrm{Au} @ \mathrm{TiO}_{2}$ is achieved within 40 minutes of irradiation [146].

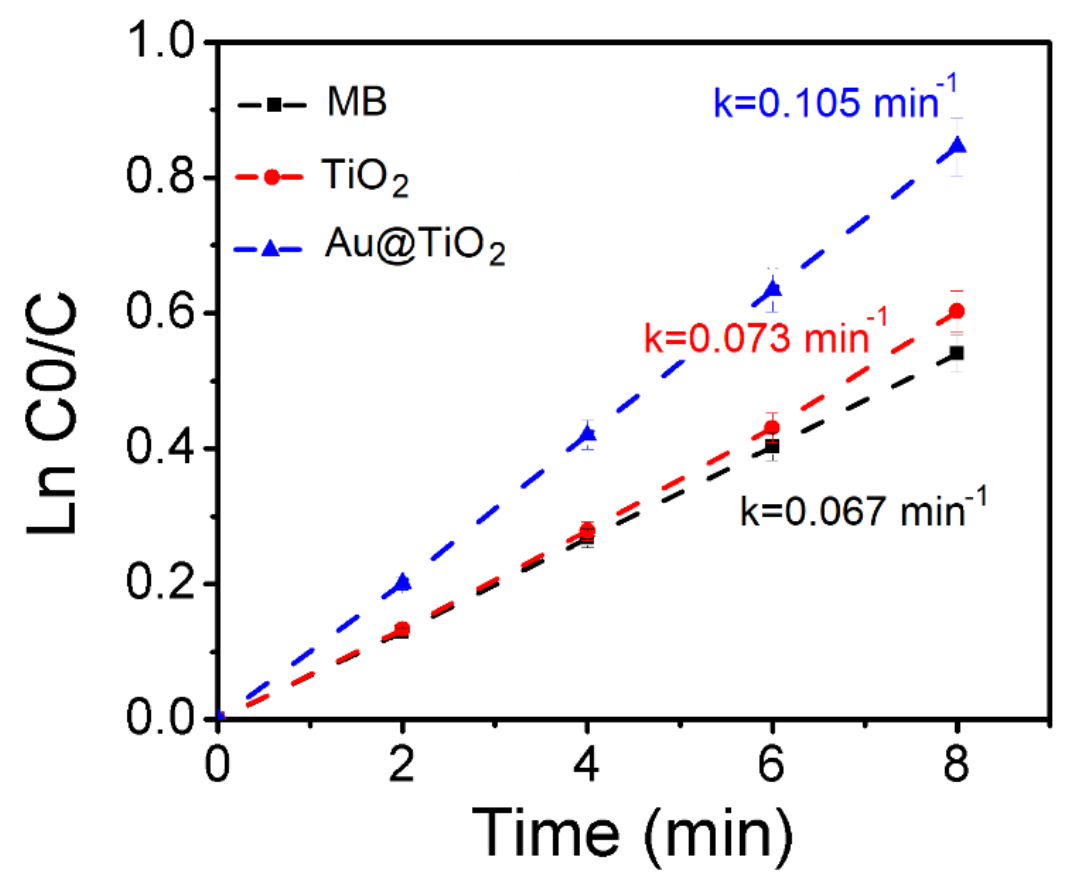

Figure 6.6. Reaction rate of $\mathrm{MB}$ using X-rays in the presence of $\mathrm{TiO}_{2}{ }^{(6.5 \mathrm{~nm})}$ and $\mathrm{Au}_{\mathrm{TiO}} \mathrm{TiO}_{2}{ }^{(6.5 \mathrm{~nm})-}$ NaOH-10\% at a concentration of $1 \mathrm{mg} / \mathrm{ml}$. Irradiation carried out at $50 \mathrm{kV}$ and a dose rate of 35 Gy/min. 


\subsubsection{Influence of $\mathrm{X}$-rays energy and concentration in $\mathrm{Au}_{0} \mathrm{TiO}_{2}$ radiosensitization.}

A study of $\mathrm{Au} @ \mathrm{TiO}_{2}$ nanocomposites radiosensitization at two different X-ray energies and concentrations of the nanocomposites in the dye solutions is shown in Figure 6.7. The results showed that MB reaction without particles does not change with the X-ray output voltage since the dose rate was fixed at voltages of $50 \mathrm{kV}$ and $225 \mathrm{kV}$. Nonetheless, in the presence of $\mathrm{Au} @ \mathrm{TiO}_{2}$ nanocomposites, a minimum and a maximum increase of $43.6 \%$ and $65.3 \%$ in the reaction rate was obtained when $\mathrm{Au} @ \mathrm{TiO}_{2}{ }^{(6.5 \mathrm{~nm})-\mathrm{NaOH}-10 \%}$ was added at a concentration of $0.2 \mathrm{mg} / \mathrm{ml}$ and 1 $\mathrm{mg} / \mathrm{ml}$ to the $\mathrm{MB}$ solutions respectively. At the selected voltages of $50 \mathrm{kV}$ and $225 \mathrm{kV}$, mean photon energies of $\sim 27 \mathrm{KeV}$ and $\sim 70 \mathrm{KeV}$ were observed according to the software SpekCalc, a tool that simulates X-ray energy spectra. At these energies, the absorption coefficient $\left(\mu_{\mathrm{en}} / \rho\right)$ of $\mathrm{Au} @ \mathrm{TiO}_{2}$ is $8.46 \mathrm{~cm}^{2} / \mathrm{g}$ for $27 \mathrm{KeV}$ and $0.14 \mathrm{~cm}^{2} / \mathrm{g}$ for $70 \mathrm{KeV}$ (NIST). The higher absorption coefficient of the nanocomposites at a voltage of $50 \mathrm{kV}$ compared to that at $225 \mathrm{kV}$ may explain the slightly higher reaction rates found at $50 \mathrm{kV}$ at either $0.2 \mathrm{mg} / \mathrm{ml}$ or $1 \mathrm{mg} / \mathrm{ml}$. Regarding Au nanoparticle concentration it has been found that, at nanomolar concentrations, radiosensitization increases linearly until reaching a plateau where the production of hydroxyl radicals does not longer vary with the amount of Au nanoparticles in solution [118,149]. 

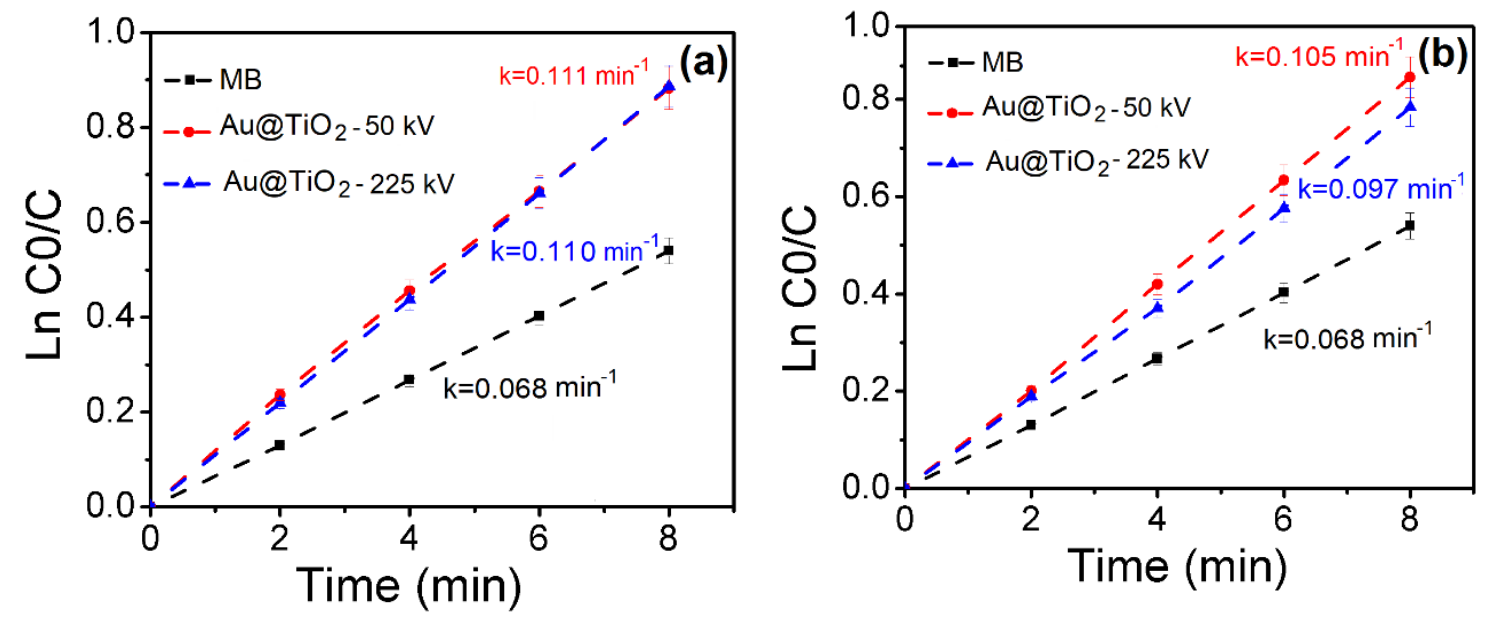

Figure 6.7. $\mathrm{MB}$ degradation in the presence of $\mathrm{Au} @ \mathrm{TiO}_{2}(6.5 \mathrm{~nm})-\mathrm{NaOH}-10 \%$ nanocomposites at concentration of a) $0.2 \mathrm{mg} / \mathrm{ml}$ nanocomposites concentration b) $1 \mathrm{mg} / \mathrm{ml}$. Beam voltages: 50 and $225 \mathrm{kV}$ using a dose rate of $35 \mathrm{~Gy} / \mathrm{min}$.

\subsubsection{Radiosensitization analysis of $\mathrm{Au} @ \mathrm{TiO}_{2}$ Nanocomposites with different particle size support.}

The influence of the support particle size $\left(6.5\right.$ or $\left.21.6 \mathrm{~nm} \mathrm{TiO}_{2}\right)$ on the radiosensitization of $\mathrm{Au} @ \mathrm{TiO}_{2}$ nanocomposites was investigated by adding $\mathrm{Au} @ \mathrm{TiO}_{2}{ }^{(6.5 \mathrm{~nm})-\mathrm{NaOH}-10 \%}$ and $\mathrm{Au} @ \mathrm{TiO}_{2}{ }^{(21.6}$ nm)-NaOH-10\% to MB and following its evolution with irradiation time. The results in Figure 6.8 show that $\mathrm{Au}$ supported on $6.5 \mathrm{~nm} \mathrm{TiO}_{2}$ lead to a greater $\mathrm{MB}$ reaction rate by $19.4 \%$ with respect to $\mathrm{Au}$ supported on $21.6 \mathrm{~nm}$ titania, indicating that the size of the $\mathrm{TiO}_{2}$ support has a significant effect for radiosensitization purposes. When the particle size of $\mathrm{TiO}_{2}$ decreases the surface area increases, leading to a larger amount of active sites that facilitate the interaction of the titania with the MB in solution [119]. The size of Au may also play a role in radiosensitization effects. To date, experiments have been carried out using Au nanoparticles with a mean particle size ranging from $\sim 2 \mathrm{~nm}$ to $\sim 50 \mathrm{~nm}$ [3]. Results have shown that smaller Au nanoparticles produce higher levels of 
reactive species. Furthermore, Au particles with a mean particle size of $2 \mathrm{~nm}$ emit a larger fraction of Auger and delta electrons than bigger particles [18].

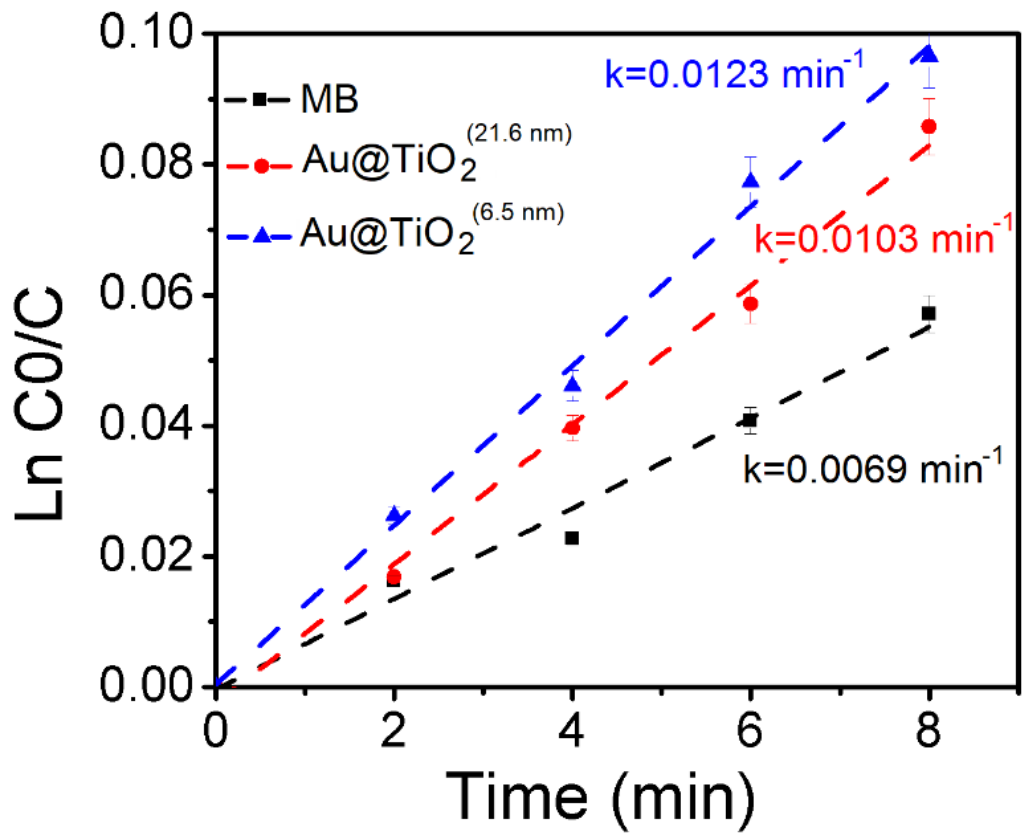

Figure 6.8. $\mathrm{MB}$ degradation in the presence of $\mathrm{Au} @ \mathrm{TiO}_{2}{ }^{(6.5 \mathrm{~nm})-\mathrm{NaOH}-10 \%}$ and $\mathrm{Au} @ \mathrm{TiO}_{2}{ }^{(21.6 \mathrm{~nm})-\mathrm{NaOH}-}$ $10 \%$ at a concentration of $0.2 \mathrm{mg} / \mathrm{ml}$. Irradiation carried out using a dose rate of $3 \mathrm{~Gy} / \mathrm{min}$ and a voltage of $225 \mathrm{kV}$.

\subsubsection{Effect of Au loading in MB radiosensitization.}

Results reported in Figure 6.9 show radiosensitization of $\mathrm{Au} @ \mathrm{TiO}_{2}{ }^{(21.6 \mathrm{~nm})}$ at loadings of $2 \%$ and $10 \%$. The reaction rate of $\mathrm{MB}$ with the nanocomposites having a $\mathrm{Au}$ loading of $2 \%$ and $10 \%$ increased by $33.0 \%$ and $49.0 \%$ respectively. This indicates that presence of Au nanoparticles at higher loadings, increases the number of reactive species in the water, leading to a faster degradation rate, reflected an increase on the reaction rate constant of the dye. Interestingly, when $\mathrm{Au} @ \mathrm{TiO}_{2}$ nanocomposites are activated using UV or visible light, Au with a loading higher than $2 \%$ could diminish their photocatalytic activity. [151]. A Au loading above this threshold causes light absorption by $\mathrm{Au}$ rather than $\mathrm{TiO}_{2}$, leading to poor catalytic performance [151]. This behavior 
was not observed when $\mathrm{Au} @ \mathrm{TiO}_{2}$ nanocomposites were irradiated with X-rays. The interaction of photons with energies between $\sim 10$ to $500 \mathrm{KeV}$ with $\mathrm{Au}$ leads predominantly to the photoelectric effect and an increase in their concentration in solution increases the production of photoelectrons, Auger electrons, and secondary X-rays, accelerating dye degradation. Au @ $\mathrm{TiO}_{2}$ showed an overall increase of $\mathrm{MB}$ reaction rate at all experimental conditions regardless of their different loading, concentration and multiple irradiation conditions as shown in Table 6.1. Summary of MB reaction rates in the presence of $\mathrm{TiO} 2$ and $\mathrm{Au} @ \mathrm{TiO} 2$ nanocomposites, where a summary of the reaction rate constants is presented. Based on the results it is concluded that the $\mathrm{Au} @ \mathrm{TiO}_{2}$ nanocomposites synthesized with X-rays radiolytic synthesis are a suitable alternative for radiosensitization applications [146].

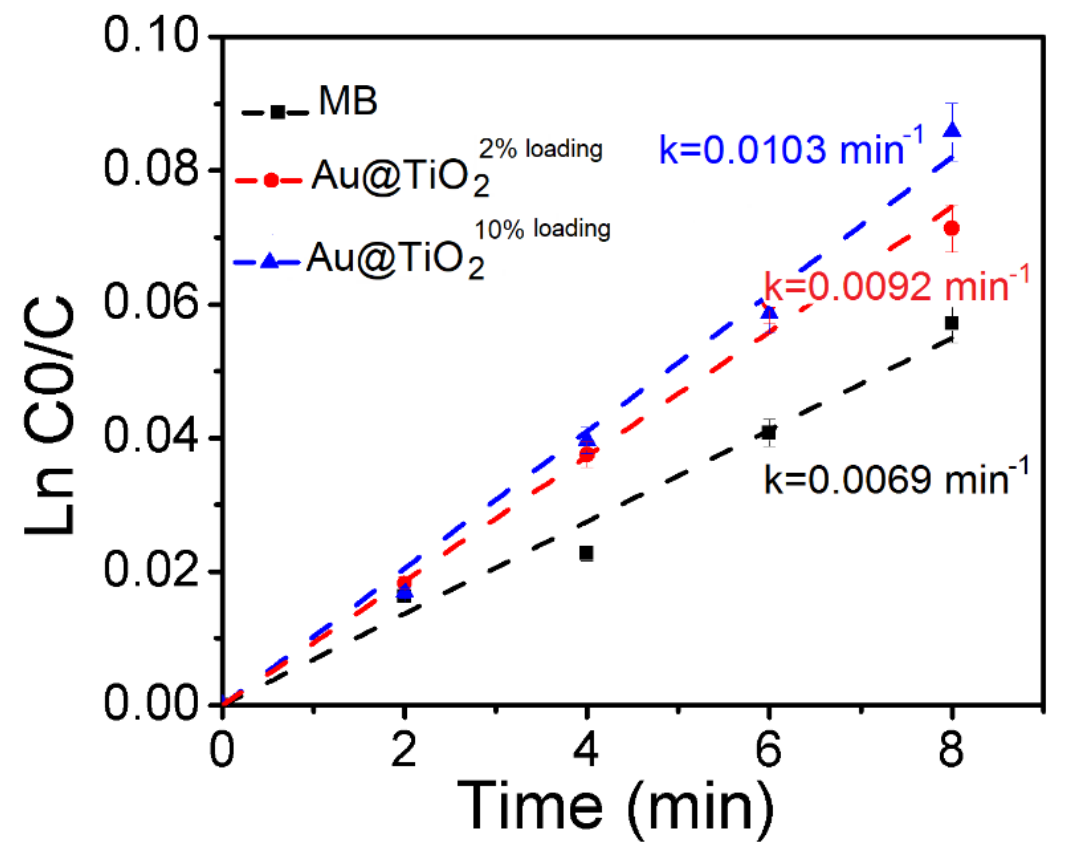

Figure 6.9. $\mathrm{MB}$ degradation in the presence of $\mathrm{Au} @ \mathrm{TiO}_{2}^{(21.6 \mathrm{~nm})-\mathrm{NaOH}-10 \%}$ and $\mathrm{Au} @ \mathrm{TiO}_{2}{ }^{(21.6 \mathrm{~nm})-}$ $\mathrm{NaOH}-2 \%$ at a concentration of $0.2 \mathrm{mg} / \mathrm{ml}$. Irradiation carried out using a dose rate of $3 \mathrm{~Gy} / \mathrm{min}$ and a voltage of $225 \mathrm{kV}$. 
Table 6.1. Summary of $\mathrm{MB}$ reaction rates in the presence of $\mathrm{TiO}_{2}$ and $\mathrm{Au} @ \mathrm{TiO}_{2}$ nanocomposites.

\begin{tabular}{|c|c|c|c|c|}
\hline Sample & Voltage & Concentration & $\begin{array}{c}\text { Dose } \\
\text { rate } \\
\text { (Gy/min) }\end{array}$ & $\begin{array}{c}\text { Reaction rate } \\
\text { constant (min }\end{array}$ \\
\hline $\mathrm{MB}$ & $50 \mathrm{kV}$ & $50 \mathrm{uM}$ & 35 & $0.067 \pm 0.003$ \\
\hline $\begin{array}{c}\mathrm{Au} @ \mathrm{TiO}_{2}{ }^{(6.5 \mathrm{~nm})-\mathrm{NaOH}-} \\
10 \%\end{array}$ & $50 \mathrm{kV}$ & $0.2 \mathrm{mg} / \mathrm{ml}$ & 35 & $0.111 \pm 0.005$ \\
\hline $\begin{array}{c}\mathrm{Au} @ \mathrm{TiO}_{2}^{(21.6 \mathrm{~nm})-\mathrm{NaOH}-} \\
10 \%\end{array}$ & $50 \mathrm{kV}$ & $0.2 \mathrm{mg} / \mathrm{ml}$ & 35 & $0.103 \pm 0.005$ \\
\hline $\begin{array}{c}\mathrm{Au} @ \mathrm{TiO}_{2}{ }^{(6.5 \mathrm{~nm})-\mathrm{Urea}-} \\
10 \%\end{array}$ & $50 \mathrm{kV}$ & $0.2 \mathrm{mg} / \mathrm{ml}$ & 35 & $0.074 \pm 0.005$ \\
\hline $\mathrm{TiO}_{2}{ }^{(6.5 \mathrm{~nm})}$ & $50 \mathrm{kV}$ & $0.2 \mathrm{mg} / \mathrm{ml}$ & 35 & $0.043 \pm 0.004$ \\
\hline $\mathrm{TiO}_{2}{ }^{(6.5 \mathrm{~nm})}$ & $50 \mathrm{kV}$ & $1 \mathrm{mg} / \mathrm{ml}$ & 35 & $0.073 \pm 0.001$ \\
\hline $\begin{array}{c}\mathrm{Au} @ \mathrm{TiO}_{2}{ }^{(6.5 \mathrm{~nm})-\mathrm{NaOH}-} \\
10 \%\end{array}$ & $50 \mathrm{kV}$ & $1 \mathrm{mg} / \mathrm{ml}$ & 35 & $0.105 \pm 0.009$ \\
\hline $\mathrm{MB}_{10 \%}$ & $225 \mathrm{kV}$ & $50 \mathrm{uM}$ & 35 & $0.068 \pm 0.002$ \\
\hline $\begin{array}{c}\mathrm{Au} @ \mathrm{TiO}_{2}{ }^{(6.5 \mathrm{~nm})-\mathrm{NaOH}-} \\
10 \%\end{array}$ & $225 \mathrm{kV}$ & $0.2 \mathrm{mg} / \mathrm{ml}$ & 35 & $0.110 \pm 0.003$ \\
\hline $\begin{array}{c}\mathrm{Au} @ \mathrm{TiO}_{2}{ }^{(6.5 \mathrm{~nm})-\mathrm{NaOH}-} \\
10 \%\end{array}$ & $225 \mathrm{kV}$ & $1 \mathrm{mg} / \mathrm{ml}$ & 35 & $0.097 \pm 0.005$ \\
\hline $\mathrm{MB}^{2}$ & $225 \mathrm{kV}$ & $50 \mathrm{uM}$ & 3 & $0.0069 \pm 0.0002$ \\
\hline $\begin{array}{c}\mathrm{Au} @ \mathrm{TiO}_{2}{ }^{(6.5 \mathrm{~nm})-\mathrm{NaOH}-} \\
10 \%\end{array}$ & $225 \mathrm{kV}$ & $0.2 \mathrm{mg} / \mathrm{ml}$ & 3 & $0.0123 \pm 0.0005$ \\
\hline $\begin{array}{c}\mathrm{Au} @ \mathrm{TiO}_{2}{ }^{(21.6 \mathrm{~nm})-\mathrm{NaOH}-} \\
10 \%\end{array}$ & $225 \mathrm{kV}$ & $0.2 \mathrm{mg} / \mathrm{ml}$ & 3 & $0.0103 \pm 0.0002$ \\
\hline $\begin{array}{c}\mathrm{Au} @ \mathrm{TiO}_{2}{ }^{(21.6 \mathrm{~nm})-\mathrm{NaOH}-} \\
2 \%\end{array}$ & $225 \mathrm{kV}$ & $0.2 \mathrm{mg} / \mathrm{ml}$ & 3 & $0.0092 \pm 0.0009$ \\
\hline
\end{tabular}

\subsubsection{Radiosensitization of $\mathrm{Au} @ \mathrm{TiO}_{2}$ Nanocomposites on M. aeruginosa.}

Radiosensitization of $\mathrm{TiO}_{2}$ and $\mathrm{Au} @ \mathrm{TiO}_{2}$ was evaluated on $\mathrm{M}$. aeruginosa cells and results are shown in Figure 6.10. Before irradiation (Figure 6.10a, Figure 6.10c and Figure 6.10e). The cells in the absence of particles show a spherical morphology. In the absence of irradiation, nanoparticle intake slightly increased the diameter of the cells from $3.0 \mu \mathrm{m}$ to $3.1 \mu \mathrm{m}$ and $3.2 \mu \mathrm{m}$ when $\mathrm{TiO}_{2}$ and $\mathrm{Au} @ \mathrm{TiO}_{2}$ were added to the cells respectively. The addition of nanomaterials prior to irradiation also caused cell deformation, evidenced by an elongated cell shape after nanoparticle 
absorption. The cells structure after irradiation (Figure 6.10b, Figure 6.10d, and Figure 6.10f), show irreversible damage. The interaction of cells with X-rays caused damage to the mucilaginous external layer of the cell in the presence and absence of $\mathrm{TiO}_{2}$ and $\mathrm{Au} @ \mathrm{TiO}_{2}$ nanoparticles. Nonetheless, when the cells are irradiated with the $\mathrm{Au} @ \mathrm{TiO}_{2}$, the damage intensifies causing cell deformation and a significant loss of their cellular content [47,109]. A higher level of physical damage is observed when $\mathrm{Au} @ \mathrm{TiO}_{2}$ are used as radiosensitizers since $\sim$ one of ten cells remains undamaged. Furthermore, SEM analysis showed that some of the particles traveled to the inside of the cells through the pores of the cells, causing destruction of the algae from the inside disrupting cell physical and chemical processes[146].

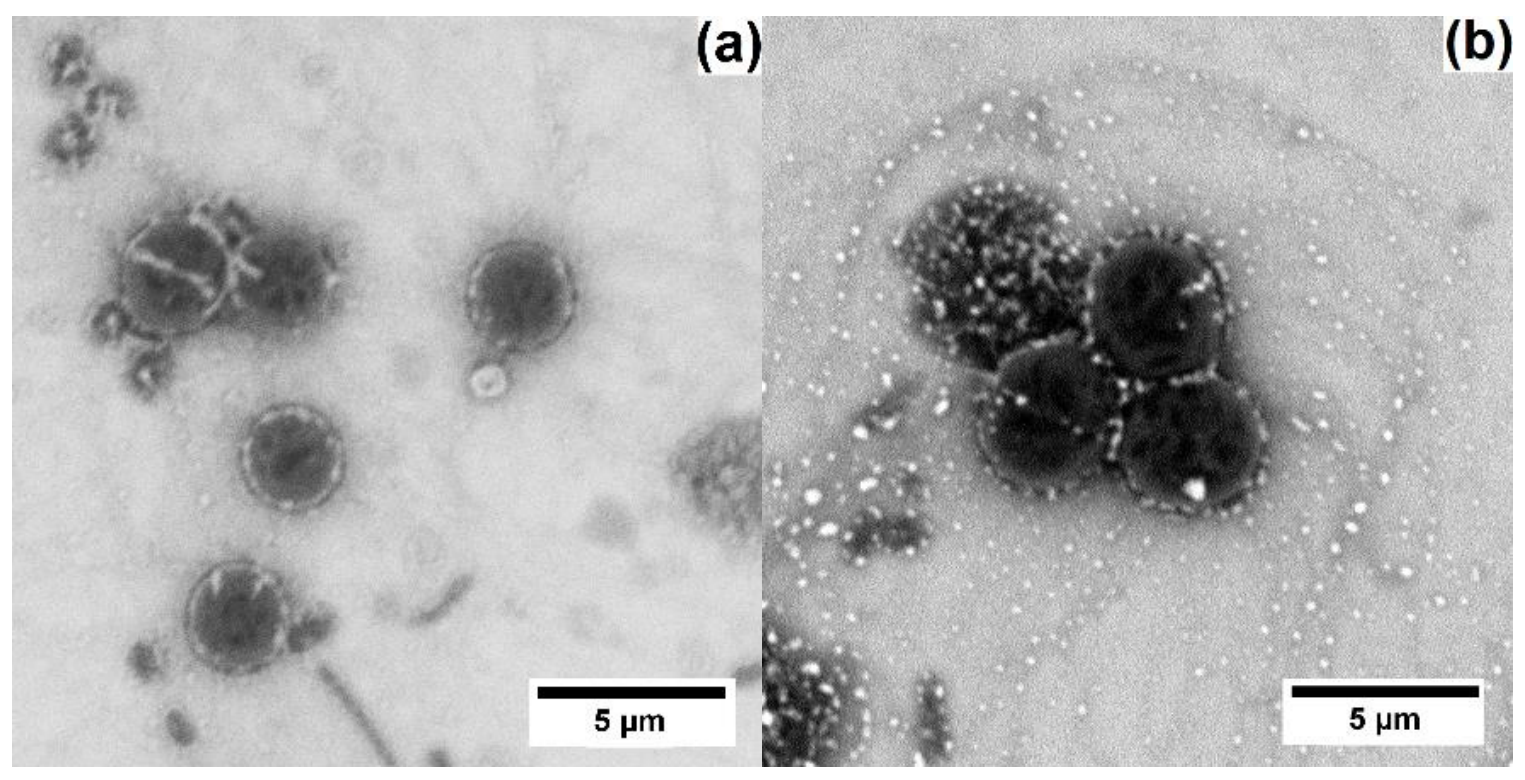



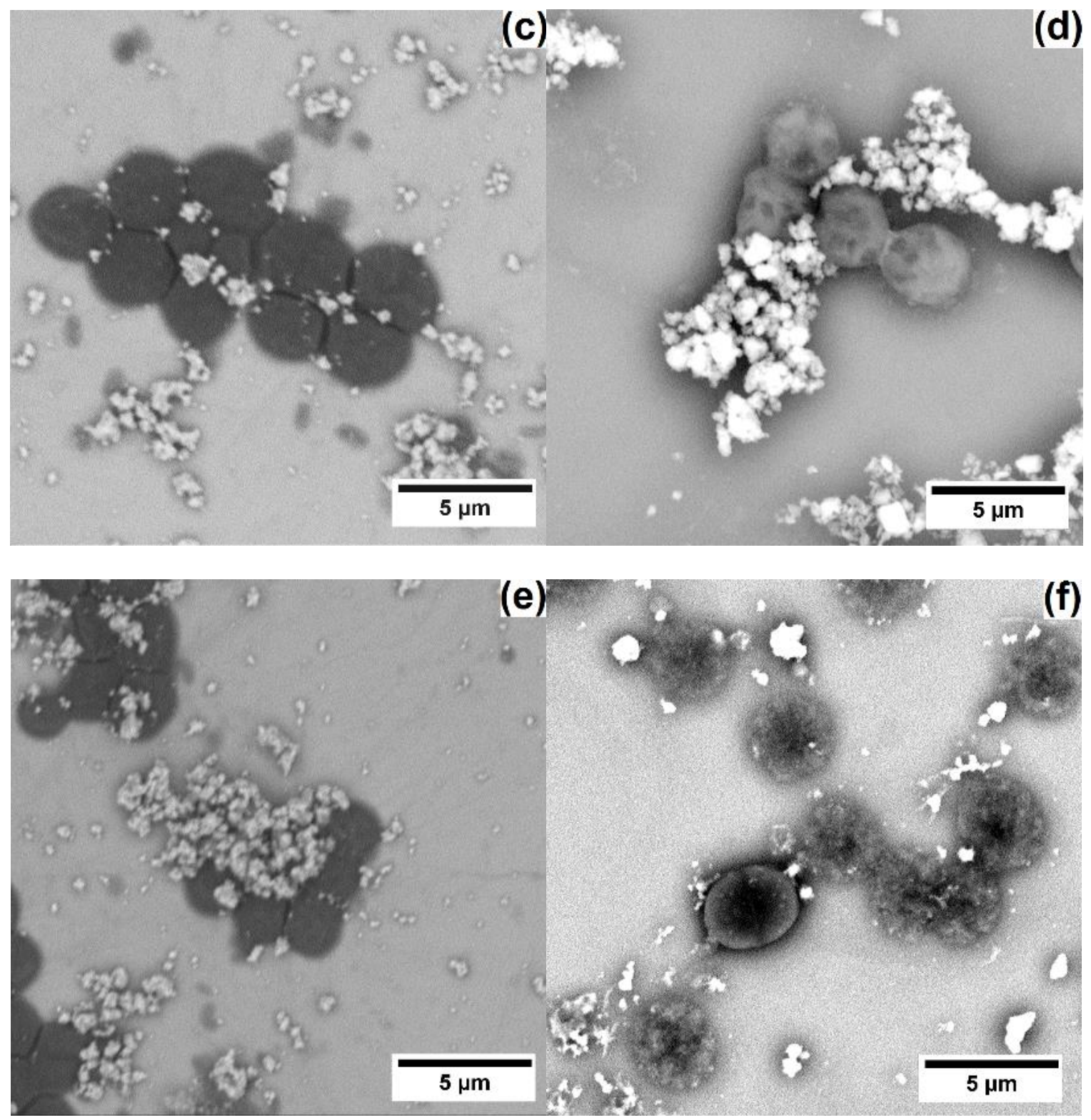

Figure 6.10. $\mathrm{SEM}$ images showing the interaction of $\mathrm{TiO}_{2}$ and $\mathrm{Au} @ \mathrm{TiO}_{2}$ with $\mathrm{M}$. aeruginosa. a) cells prior irradiation b) cells damage after irradiation c) cells interaction with $\mathrm{TiO}_{2}$ d) cells irradiated in the presence of $\mathrm{TiO}_{2}$ e) cells interaction with $\mathrm{Au} @ \mathrm{TiO}_{2}$ f) cells irradiated in the presence of $\mathrm{Au} @ \mathrm{TiO}_{2}$. Cells irradiated with a dose of $6 \mathrm{kGy}$.

Irradiation also showed a decrease in the chlorophyll production of the cells, an important parameter, since $\mathrm{M}$. aeruginosa uses light to grow and reproduce. UV-Vis results are shown in Figure 6.11. The patterns were analyzed at a wavelength of $679 \mathrm{~nm}$, absorbance at this wavelength 
is related to chlorophyll production and cells content in suspension [152]. After irradiation, a continuous decrease of the absorbance at $679 \mathrm{~nm}$ is observed every day. After 3 days of irradiation both $\mathrm{TiO}_{2}$ and $\mathrm{Au} @ \mathrm{TiO}_{2}$ showed a significant decrease in absorbance of $26.8 \%$ and $38.6 \%$ respectively when compared with the cells irradiated at $6 \mathrm{kGy}$, whereas after 5 days of irradiation, the difference in chlorophyll production was no longer observed since all the irradiated samples showed similar absorbance values. In conclusion, both SEM and UV-Vis results showed superior cell damage in the presence of $\mathrm{Au} @ \mathrm{TiO}_{2}$ as radiosensitizers.

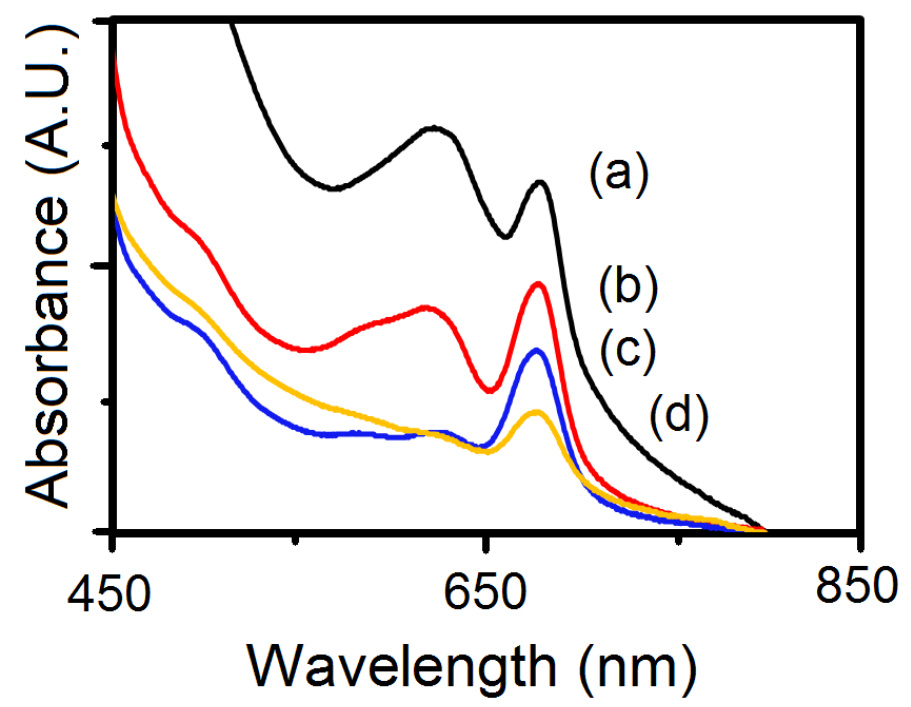

Figure 6.11. UV-Vis spectra of $M$. aeruginosa a) Nonirradiated cells b) Irradiated cells c) Irradiated cells in the presence of $\mathrm{TiO}_{2}$ d) irradiated cells in the presence of $\mathrm{Au} @ \mathrm{TiO}_{2}$. Measurements were taken 3 days after irradiation at a dose of $6 \mathrm{kGy}$.

The results presented in this section have been adapted from a manuscript entitled: "X-ray radiation enhancement of gold-TiO 2 nanocomposites". Published in applied surface science. 


\subsection{Conclusions.}

In this chapter, the potential of $\mathrm{Au} @ \mathrm{TiO}_{2}$ nanocomposites as radiosensitizers was discussed based on the light of the results that demonstrated their physical, chemical and biological enhancement. Analysis on parameters such as surface chemistry, support particle size, activation voltage, dose rate, and $\mathrm{Au}$ loading were evaluated, in order to evidence the effects of $\mathrm{Au} @ \mathrm{TiO}_{2}$ on $\mathrm{MB}$ reaction rate constant and their corresponding dose enhancement. Au@ $\mathrm{TiO}_{2}$ nanocomposites synthesized in the presence of $\mathrm{NaOH}$ showed higher radiosensitization over nanocomposites fabricated with urea by $50 \%$. Moreover, at an operating voltage of $225 \mathrm{kV}$, Au supported on $6.5 \mathrm{~nm}$ titania particles lead to a higher $\mathrm{MB}$ decomposition rate when compared to that with $\mathrm{Au}$ deposited on $\mathrm{TiO}_{2}$ with a mean particle size of $21.6 \mathrm{~nm}$. These results have been also observed in photocatalytic applications of $\mathrm{TiO}_{2}$, where a high number of active sites are found when the particle size of the nanomaterial decreases. Results also showed that when the voltage is modified, keeping the dose rate constant, it did not affect the reaction rate constant of MB. In addition, when MB was irradiated using a dose rate of $\sim 3 \mathrm{~Gy} / \mathrm{min}$, its reaction rate constant was $\sim 10$ times lower than that at a dose rate of 35 Gy/min. However, at lower dose rates the percentage of increase in the reaction rate of MB in the presence of $\mathrm{Au} @ \mathrm{TiO}_{2}{ }^{(6.5 \mathrm{~nm})-\mathrm{NaOH}-10 \%}$ was $78 \%$. These results are in agreement with the literature, since at low irradiation dose rates, ROS show lower recombination rates, increasing the overall radiosensitization effect. $\mathrm{Au} @ \mathrm{TiO}_{2}$ radiosensitization was also tested in a biological scenario by evidencing enhanced cell damage of M. aeruginosa. This experiment demonstrated promising antibacterial properties of $\mathrm{Au} @ \mathrm{TiO}_{2}$, a feature could be important in water treatment applications. Finally, this work confirmed X-ray radiolysis as a feasible approach for the synthesis of materials intended for medical applications, this synthesis method uses clean solvents such as water and isopropanol, and produces low to none by-products on the surface nanomaterial, this is important 
because an excess of chemical agents on the surface of nanoparticles could hinder their physical and chemical properties. 


\section{Chapter 7: Comparison of $\mathrm{Au} @ \mathrm{TiO}_{2}$ radiosensitization with other metal oxides systems at the megavoltage range}

\subsection{Introduction.}

The nanomaterials evaluated as radiosensitizers at a megavoltage range in this chapter include $\mathrm{TiO}_{2}$, $\mathrm{ZnO}, \mathrm{HfO}_{2}$, which are known to be wide bandgap semiconductors [153,154]. Radiosensitization of these metal oxides is based on the interaction of the nanomaterial with X-rays. When semiconductors interact with a photon with higher energy than its bandgap, electron-holes pairs are produced. Electron-hole pairs will interact with the media causing an increment of ROS in solution, potentially increasing the radiosensitization effect in solution [22,39]. Specifically, $\mathrm{ZnO}$ is a semiconductor used in different electronic applications such as ferromagnetism, optoelectronics, transducers technology and solar cell harvesting [155]. ZnO has a band gap of $\sim 3.3 \mathrm{eV}$ and relevant properties for catalysis include: mild to low toxicity, corrosion resistance and environmental sustainability [155]. On the other hand, $\mathrm{HfO}_{2}$ is a metal oxide with a high melting point $\left(\sim 2700{ }^{\circ} \mathrm{C}\right)$, high dielectric constant, and a wide band gap of $5 \mathrm{eV}$. Because $\mathrm{HfO}_{2}$ is composed of $\mathrm{Hf}$, a high $\mathrm{Z}$ element, it has been used for electronics and target therapy applications $[153,156]$. Herein, the radiosensitization of the metal oxides was evidenced by monitoring the decomposition of $\mathrm{MB}$ in the presence of the nanomaterial. Furthermore, $\mathrm{Au} @ \mathrm{ZnO}$ and $\mathrm{Au} @ \mathrm{HfO}_{2}$ were synthesized using X-ray radiolytic synthesis and their radiosensitization was compared to $\mathrm{Au} @ \mathrm{TiO}_{2}$. In this chapter, the potential of $\mathrm{Au} @ \mathrm{TiO}_{2}$ and other $\mathrm{Au} @$ oxides as radiosensitizers at energies of interest in radiation therapy $(\mathrm{MeV}$ range) is evidenced. This opens up the possibility for future research of in-vitro and in-vivo applications of these materials in relevant fields of medicine and catalysis. 


\subsection{Experimental Procedure.}

\subsubsection{Materials and Reagents.}

$\mathrm{HfO}_{2}$ with a mean particle size of $75 \mathrm{~nm}$ and $\mathrm{ZnO}$ with a particle size of $25.7 \mathrm{~nm}$ were purchased from US Research nanomaterials Inc. Synthesis of $\mathrm{HfO}_{2}$ nanoparticles with particle size $\sim 8.4 \mathrm{~nm}$ was carried out using Hafnium tetrachloride $\left(\mathrm{HfCl}_{4}\right)$ from Across Organics. Finally, Au supported oxides were synthesized using Chloroauric acid $\left(\mathrm{HAuCl}_{4} \cdot 3 \mathrm{H}_{2} \mathrm{O}, \geq 99.9 \%\right.$ trace metal basis), isopropanol $\left(\mathrm{C}_{3} \mathrm{H}_{8} \mathrm{O}, \geq 99.7 \%\right)$, and sodium hydroxide $(\mathrm{NaOH})$ obtained from Sigma-Aldrich. Deionized water (DI) from a Millipore Direct QTM 3 UV purification system was used to prepare all aqueous solutions.

\subsubsection{X-rays radiolytic synthesis of Au@ $\mathrm{ZnO}$ and $\mathrm{Au} @ \mathrm{HfO}_{2}$.}

X-ray radiolytic synthesis of $\mathrm{Au} @ \mathrm{ZnO}$ and $\mathrm{Au} @ \mathrm{HfO}_{2}$ were synthesized in the presence of urea, using a similar protocol as the one developed for the synthesis of $\mathrm{Au} @ \mathrm{TiO}_{2}$ nanocomposites and explained in detail in chapter 4. For this purpose, an aqueous solution of $\mathrm{HAuCl}_{4}(2 \mathrm{mM})$ was prepared and urea powder was added to reach a concentration of $0.42 \mathrm{M}$. Subsequently, the supports in a concentration of $4 \mathrm{mg} / \mathrm{ml}$ were added to the solution, leading to a $10 \mathrm{wt} \%$ nominal Au loading onto the support. Subsequently a heating process at $80{ }^{\circ} \mathrm{C}$ under continuous stirring for 1 hour. Afterward, the solution was washed to remove unreacted species. Radiolytic synthesis was carried out using in a Precision X-ray irradiator equipped with a W target, using a voltage of $225 \mathrm{kV}$ and a current of $13.3 \mathrm{~mA}$. The irradiation process was performed reaching a total absorbed dose of $7.26 \mathrm{kGy}$ and a dose rate of $127 \mathrm{~Gy} / \mathrm{min}$. The as-synthesized materials were named as $\mathrm{Au} @ \mathrm{ZnO}$ and Au@ $\mathrm{HfO}_{2}{ }^{75 \mathrm{~nm}}$. 


\subsubsection{Hydrothermal Synthesis of $\mathrm{HfO}_{2}$.}

Synthesis of $\mathrm{HfO}_{2}$ with a mean particle size of $8.4 \mathrm{~nm}$, was carried out following a procedure found in the literature [157]. First, a $25 \mathrm{ml}$ stock solution of $\mathrm{HfCl}_{4}$ with a concentration of $0.1 \mathrm{M}$ was prepared. Then, $25 \mathrm{ml}$ of $\mathrm{NaOH}$ solution $(0.4 \mathrm{M})$ was slowly added to the $\mathrm{HfCl}_{4}$ stock solution, this process was made in a dropwise manner. Afterwards, the solution containing $\mathrm{HfCl}_{4}$ and $\mathrm{NaOH}$ was stirred for 6 hours, during the stirring process a white precipitate of hafnium hydroxide was produced. This precipitate was centrifuged and washed with DI water at $9000 \mathrm{rpm}$ for 10 minutes. Then, the precipitate was dried in an oven at $100{ }^{\circ} \mathrm{C}$ for $3 \mathrm{~h}$, followed by calcination at $500{ }^{\circ} \mathrm{C}$ for 2 hours. The white pellet was collected and ground to a fine powder using a mortar and pestle. In order to differentiate the samples of $\mathrm{HfO}_{2}$ with different particle size, they were identified as $\mathrm{HfO}_{2}{ }^{8.4 \mathrm{~nm}}$ and $\mathrm{HfO}_{2}{ }^{75 \mathrm{~nm}}$.

\subsubsection{Characterization of metal-oxide nanoparticles.}

The particle size of metal oxides and Au@oxides was evaluated by TEM using a Zeiss Libra 120 plus operating at a voltage of $120 \mathrm{kV}$. TEM samples were prepared by dispersing the nanopowders in acetone using an ultrasonic probe. A $20 \mu \mathrm{l}$ drop of these solutions was deposited onto a formvar/carbon copper grid. The statistical analysis of the nanoparticles' particle size was made with Image J 1.5i. Crystalline structure analysis of the metal oxides and nanocomposites was investigated using a PAnalytical X'Pert Pro X-ray diffractometer with a Cu target at an operating voltage of $45 \mathrm{kV}$ and a current of $40 \mathrm{~mA}$. For this purpose, the nanopowders were initially placed onto a low-background silicon wafer and loaded onto the spinning sample stage with a low angular speed. The diffraction patterns were analyzed with the software X'pert Highscore. 


\subsubsection{Radiosensitization Assessment of metal-oxide semiconductors.}

The radiocatalytic activity of oxides and Au@ oxides was measured by monitoring the degradation of a Methylene Blue (MB) solution in the presence of the nanomaterial. For this purpose, the nanopowders in a concentration of $0.2 \mathrm{mg} / \mathrm{ml}$ were added to the aqueous solution of $\mathrm{MB}$ with a concentration of $50 \mu \mathrm{M}$. The $\mathrm{MB}$ degradation was analyzed by following the decrease of its characteristic absorbance peak at $664 \mathrm{~nm}$ with irradiation time. The spectra were collected with a Genesis 10S UV-Vis spectrophotometer. The MB solutions were irradiated in the Madison accelerator laboratory (MAL) at James Madison University using a Siemens LINAC operating at an endpoint energy of $6 \mathrm{MeV}$. The irradiation field was set to $10 \mathrm{x} 10 \mathrm{~cm}$, using a fixed dose rate of $8 \mathrm{~Gy} / \mathrm{min}$. With this experiment, the influence of the particle size in radiosensitization was evaluated on $\mathrm{TiO}_{2}$ and $\mathrm{HfO}_{2}$, and the effect of $\mathrm{Au}$ deposition onto $\mathrm{TiO}_{2}, \mathrm{ZnO}$ and $\mathrm{HfO}_{2}{ }^{75} \mathrm{~nm}$ radiosensitization was investigated. The $\mathrm{MB}$ reaction rate constants in the presence and absence of the nanopowders were obtained and used to compare their radiosensitization performance.

\subsection{Results and Discussion.}

\subsubsection{Crystalline structure of supports.}

Analysis of the XRD patterns is shown in Figure 7.1. $\mathrm{HfO}_{2}$ and $\mathrm{ZnO}$ revealed monoclinic (JCSPDS 06-0318) and wurtzite (JCPDS 5-0664) crystalline structures respectively. The crystallite size of the metal oxides was calculated using Scherrer equation, and values of $8.3 \mathrm{~nm}, 13.1 \mathrm{~nm}$, and $23.5 \mathrm{~nm}$ were obtained for $\mathrm{HfO}_{2}{ }^{(8.4 \mathrm{~nm})}$, $\mathrm{HfO}_{2}{ }^{(75 \mathrm{~nm})}$ and $\mathrm{ZnO}$ respectively. The crystallite size of metal oxides is in agreement with the particle size analysis by TEM as shown in Figure 7.2. The Au peaks in the patterns $\mathrm{Au} @ \mathrm{ZnO}$ and $\mathrm{Au} @ \mathrm{HfO}_{2}{ }^{(75 \mathrm{~nm})}$ are difficult to detect since $\mathrm{ZnO}$ and $\mathrm{HfO}_{2}$ have peaks nearby the fcc Au 2 theta positions of $38.1^{\circ}, 44.3^{\circ}$, and $64.5^{\circ}$. However, the presence of Au in the samples 
was verified with EDX and, a $\mathrm{Au} / \mathrm{ZnO}$ mass ratio of 0.06 and a mass ratio of $\mathrm{Au} / \mathrm{HfO}_{2}$ of 0.10 were found.

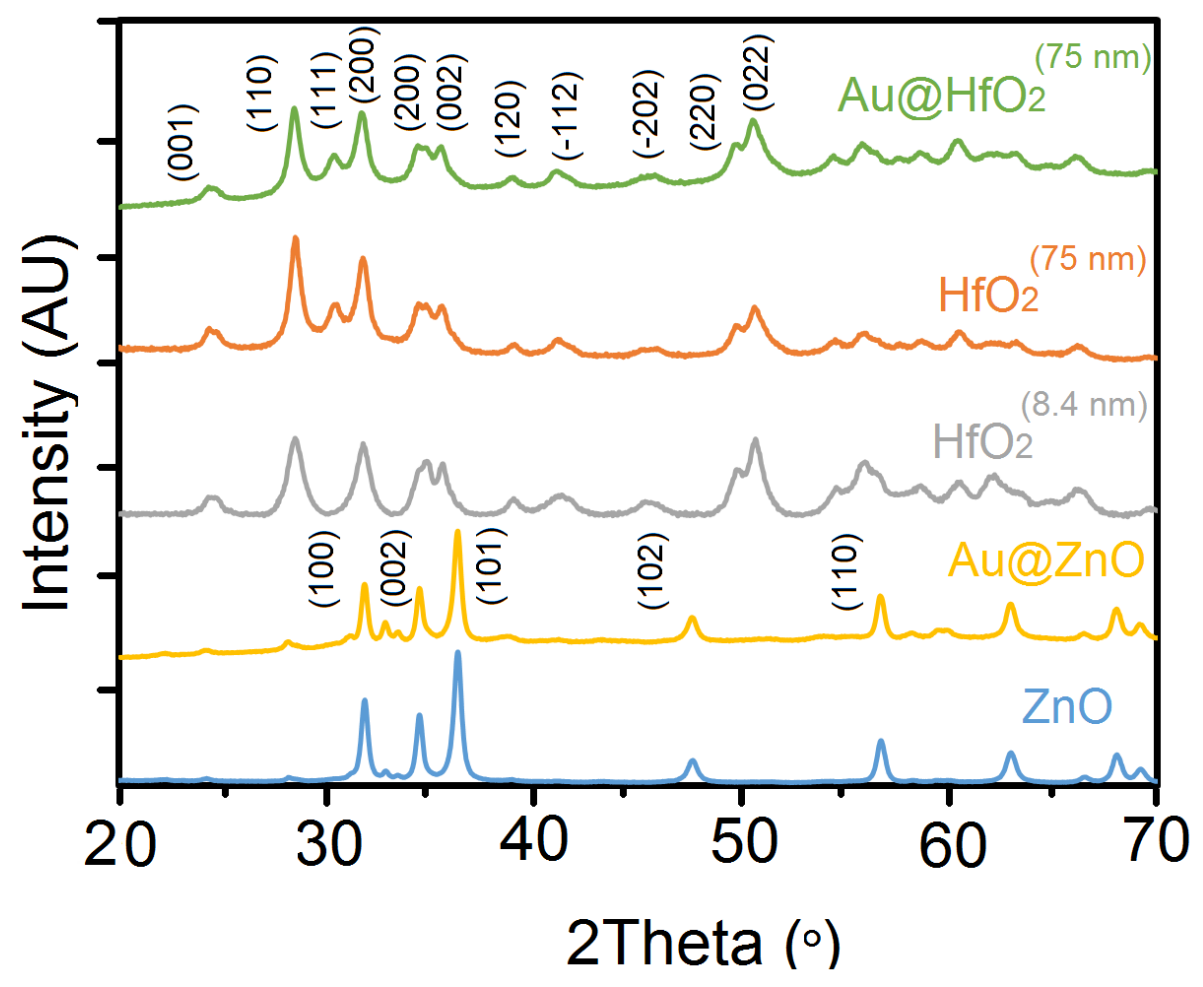

Figure 7.1. XRD Patterns of metal oxides and Au@oxides.

\subsubsection{Morphology analysis of metal oxides and Au@oxides.}

Statistical analysis of the particle's morphology by TEM showed elongated metal oxide particles (Figure 7.2). Specifically, a mean value of $25.7 \pm 9.0 \mathrm{~nm}$ was measured for $\mathrm{ZnO}$ and a mean particle size of $8.4 \pm 2.0 \mathrm{~nm}$ and $75 \pm 18.3 \mathrm{~nm}$ was found for $\mathrm{HfO}_{2}$. Interestingly, comparison of the $\mathrm{HfO}_{2}$ mean particle size obtained by TEM $(75 \mathrm{~nm})$ with the crystallite size obtained by XRD $(23.5 \mathrm{~nm})$, shows that $\mathrm{HfO}_{2}$ particles are formed of smaller crystallites evidencing their polycrystalline structure. Analysis of Au nanoparticles supported on $\mathrm{ZnO}$ nanoparticles revealed a mean particle size of $1.9 \pm 0.6 \mathrm{~nm}$, nonetheless, a few Au particles with a mean particle size of 
$10 \mathrm{~nm}$ were observed. On the other hand, Au nanoparticles supported on $\mathrm{HfO}_{2}$ presented a mean particle size of $5.3 \pm 1.3 \mathrm{~nm}$.

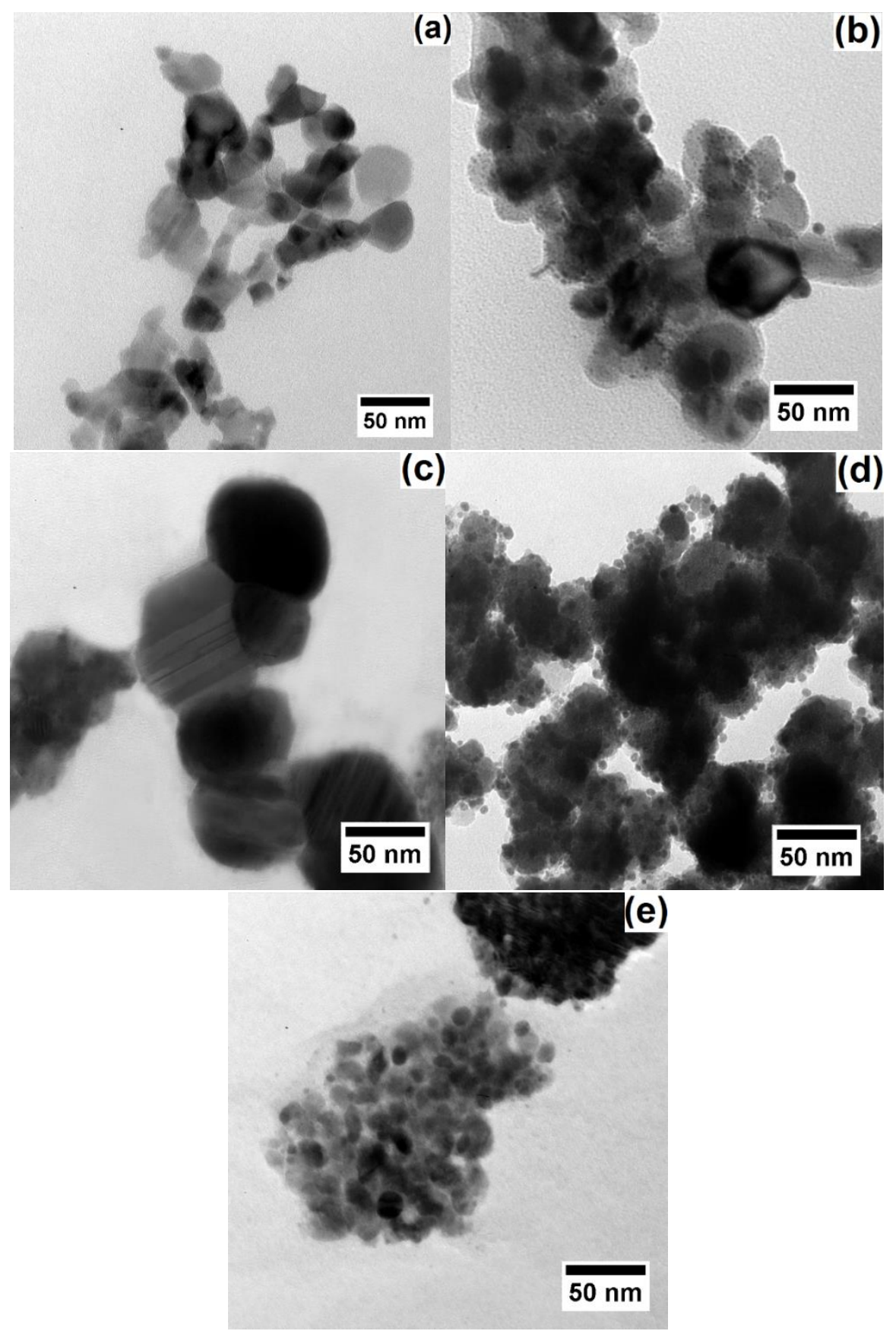

Figure 7.2. TEMs of metal oxides for a) $\mathrm{ZnO}$ b) $\mathrm{Au} @ \mathrm{ZnO}$ c) $\mathrm{HfO}_{2}{ }^{75 \mathrm{~nm}}$ d) $\mathrm{Au} @ \mathrm{HfO}_{2}{ }^{75} \mathrm{~nm}$ e) $\mathrm{HfO}_{2}{ }^{8.4 \mathrm{~nm}}$ 


\subsubsection{Radiosensitization of metal oxides and Au@oxides.}

MB reaction rate enhancement in the presence of the radiosensitizers is observed in Figure 7.3. Bare metal oxides such as $\mathrm{ZnO}, \mathrm{HfO}_{2}{ }^{8.4 \mathrm{~nm}}$ and $\mathrm{HfO}_{2}{ }^{75 \mathrm{~nm}}$ show potential as radiosensitizer with a MB degradation enhancement of $42.3 \%$ and $19.5 \%$ for $\mathrm{HfO}_{2}{ }^{8.4 \mathrm{~nm}}$ and $\mathrm{HfO}_{2}{ }^{75 \mathrm{~nm}}$, and a degradation enhancement of $19.5 \%$ for $\mathrm{ZnO}$. On the other hand, bare $\mathrm{TiO}_{2}$ with a mean particle size of $6.5 \mathrm{~nm}$ was the only oxide that showed an anti-enhancement behavior, meaning that the degradation rate of $\mathrm{MB}$ was higher in the absence of the nanocomposites. However, when Au particles were deposited on the $\mathrm{TiO}_{2}$, results showed an increase in the degradation rate of $\mathrm{MB}$ by $57.1 \%$ and $50.3 \%$ for $\mathrm{Au} @ \mathrm{TiO}_{2}{ }^{21.6 \mathrm{~nm}}$ and $\mathrm{Au} @ \mathrm{TiO}_{2}{ }^{6.5} \mathrm{~nm}$. On the other hand, $\mathrm{Au} @ \mathrm{ZnO}$ showed similar reaction rate enhancement to that with $\mathrm{ZnO}$ with a value of $20.3 \%$. Deposition of $\mathrm{Au}$ on $\mathrm{HfO}_{2}{ }^{75} \mathrm{~nm}$ also led to similar dose enhancement in $\mathrm{MB}$ to that of bare $\mathrm{HfO}_{2}$ with a value of $22.2 \%$. Low radiosensitization enhancement on $\mathrm{Au} @ \mathrm{ZnO}$ and $\mathrm{Au} @ \mathrm{HfO}_{2}{ }^{75} \mathrm{~nm}$ was attributed to the ligand chosen for radiolytic synthesis: urea. The superior enhancement of $\mathrm{Au} @ \mathrm{TiO}_{2}$ nanocomposites is due to photon absorption by the Au particles, this leads to the generation of energetic electrons which interact with the surroundings producing ROS and accelerate pollutant decomposition. These experiments also showed the importance of particle size on their response, reflected on the MB degradation rate enhancement of $\mathrm{Au} @ \mathrm{TiO}_{2}{ }^{6.5 \mathrm{~nm}-\mathrm{NaOH}}$ and $\mathrm{HfO}_{2}{ }^{8.4 \mathrm{~nm}}$. The reason behind this enhancement is a large amount of active catalytic sites present in small particles. Degradation rate enhancement due to the difference in particle size is of significant importance for $\mathrm{HfO}_{2}$ since a great difference was found between the degradation rate enhancement of $\mathrm{HfO}_{2}{ }^{75 \mathrm{~nm}}$ and $\mathrm{HfO}_{2}{ }^{8.4 \mathrm{~nm}}$ as shown in Table 7.1. Overall, the degradation of the dye is enhanced significantly in the presence of the metal oxides, due to their ability to improve the effectiveness of X-ray irradiation through different dose enhancement mechanisms known as physical and chemical enhancement. A 
summary of the radiation enhancement results obtained using a LINAC for the different materials is shown in Table 7.1.

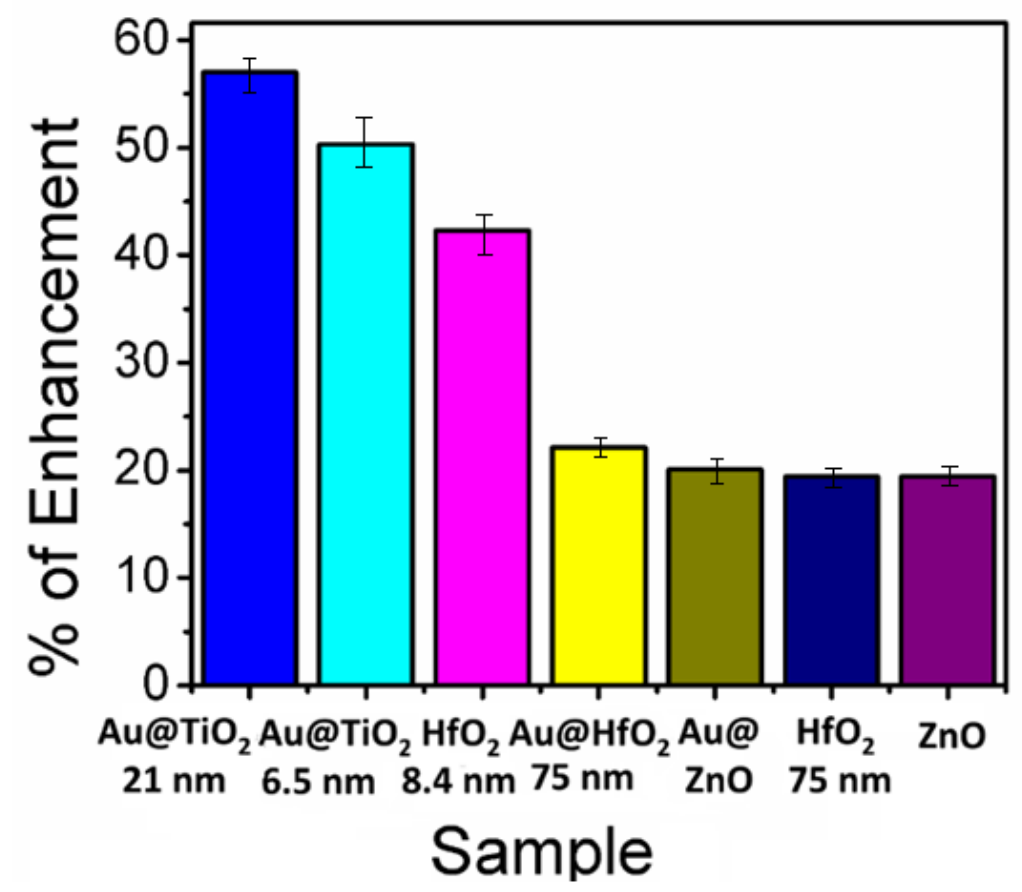

Figure 7.3. Increase of $\mathrm{MB}$ reaction rate constant in the presence of metal oxides and $\mathrm{Au} @$ metal oxides.

Table 7.1. Summary of MB degradation in the presence of different metal oxides and Au supported oxides.

\begin{tabular}{|c|c|c|c|}
\hline Sample & $\begin{array}{c}\text { Degradation } \\
\text { Rate (min }\end{array}$ & $\begin{array}{c}\text { Standard } \\
\text { deviation }\end{array}$ & $\begin{array}{c}\text { \% of } \\
\text { enhancement }\end{array}$ \\
\hline $\mathrm{MB}$ & 0.0149 & 0.003 & N/A \\
\hline $\mathrm{Au} @ \mathrm{TiO}_{2}{ }^{21.6 \mathrm{~nm}}$ & 0.0234 & 0.002 & 57.05 \\
\hline $\mathrm{Au} @ \mathrm{TiO}_{2}{ }^{6.5 \mathrm{~nm}}$ & 0.0224 & 0.003 & 50.34 \\
\hline $\mathrm{HfO}_{2}{ }^{8.4 \mathrm{~nm}}$ & 0.0212 & 0.003 & 42.28 \\
\hline $\mathrm{Au} @ \mathrm{HfO}_{2}{ }^{75} \mathrm{~nm}$ & 0.0178 & 0.002 & 22.15 \\
\hline $\mathrm{Au} @ \mathrm{ZnO}^{75 n}$ & 0.0179 & 0.002 & 20.13 \\
\hline $\mathrm{HfO}{ }^{75} \mathrm{~nm}$ & 0.0182 & 0.003 & 19.46 \\
\hline $\mathrm{ZnO}$ & 0.0178 & 0.0003 & 19.46 \\
\hline
\end{tabular}


The results presented in this section have been adapted from a manuscript entitled: "Radiocatalytic performance of oxide-based nanoparticles for targeted therapy and water remediation". Under review in the journal of radiation physics and chemistry.

\subsection{Conclusions.}

Metal oxide nanoparticles and Au@oxides were successfully tested as radiosensitizers using a LINAC with an endpoint energy of $6 \mathrm{MeV}$, a voltage used for radiation therapy purposes. The higher $\mathrm{MB}$ reaction rates found in the presence of most of the nanomaterials is due to the increase in the production of ROS generated from the interaction of X-rays with the particles. Results indicated that metal oxide composed of high $\mathrm{Z}$ elements such as $\mathrm{Au}$ and $\mathrm{Hf}$, showed superior $\mathrm{MB}$ degradation rates when compared with metal oxide containing low $\mathrm{Z}$ elements. Furthermore, nanoparticles with a small particle size such as $\mathrm{Au} @ \mathrm{TiO}_{2}{ }^{(6.5 \mathrm{~nm})-\mathrm{NaOH}}$ and $\mathrm{HfO}_{2}{ }^{8.4 \mathrm{~nm}}$, showed higher MB degradation rates when compared to larger particles $(\sim 75 \mathrm{~nm})$ by a factor of 2.2 . The reaction rate constant obtained for $\mathrm{Au} @ \mathrm{TiO}_{2}$ at dose rate of $8 \mathrm{~Gy} / \mathrm{min}\left(0.0224 \mathrm{~min}^{-1}\right)$, is consistent with the reaction rates obtained at dose rates of $3 \mathrm{~Gy} / \mathrm{min}\left(0.0123 \mathrm{~min}^{-1}\right)$ and $35 \mathrm{~Gy} / \mathrm{min}\left(0.111 \mathrm{~min}^{-1}\right)$, showing that higher dose rates will lead to a high degradation rate. This work has demonstrated the feasibility of metal oxides as radiosensitizers, a concept that can be further explored for water remediation, since results showed favorable dye decomposition, in a degradation process carried at ambient temperature and pressure, in the absence of toxic chemicals. 


\section{Chapter 8: Conclusions and Future work}

\subsection{Conclusions.}

In this work, $\mathrm{Au} @ \mathrm{TiO}_{2}$ nanocomposites were tested as radiosensitizers through the degradation of MB and enhanced M. Aeruginosa cell damage. The influence of parameters such as Au loading, $\mathrm{TiO}_{2}$ particle size, ligand of choosing, nanomaterial concentration, different irradiation voltages and dose rates on $\mathrm{Au} @ \mathrm{TiO}_{2}$ radiosensitization was evaluated. Au nanoparticles were supported on $\mathrm{TiO}_{2}$ with a mean particle of $6.5 \mathrm{~nm}$ and $21.6 \mathrm{~nm}$ in the presence of ligands such as $\mathrm{NaOH}$ and urea using radiation chemistry. The radiation enhancement generated by the nanocomposites was evidenced at various irradiation voltages of $50 \mathrm{kV}, 225 \mathrm{kV}$, and $6 \mathrm{MV}$, using concentrations of $0.2 \mathrm{mg} / \mathrm{ml}$ and 1 $\mathrm{mg} / \mathrm{ml}$, and dose rates of $3 \mathrm{~Gy} / \mathrm{min}, 8 \mathrm{~Gy} / \mathrm{min}$ and $35 \mathrm{~Gy} / \mathrm{min}$ in $\mathrm{MB}$. These variations were selected in order to investigate their effects on their performance to enable the use of $\mathrm{Au} @ \mathrm{TiO}_{2}$ nanocomposites in future in-vivo experiments. Superior radiosensitization at $225 \mathrm{kV}$ was obtained when $\mathrm{Au}$ was supported on $6.5 \mathrm{~nm}$ titania, using $\mathrm{NaOH}$ as a ligand. These results evidence that $\mathrm{TiO}_{2}$ nanoparticles with a small particle size have a high number of catalytic sites, leading to a higher decomposition rate. Furthermore, Au loading on titania (either $2 \%$ or $10 \%$ ) is a relevant parameter for radiosensitization, since a high Au loading of $10 \%$ showed an increase of MB degradation rate when compared with a $\mathrm{TiO}_{2}$ with a $2 \%$ Au loading. Interestingly, parameters such as operating voltage did not affect significantly $\mathrm{Au} @ \mathrm{TiO}_{2}$ radiosensitization. The radiosensitization performance of $\mathrm{Au} @ \mathrm{TiO}_{2}$ nanocomposites was tested using LINAC with an energy endpoint of $6 \mathrm{MeV}$ and compared with other metal oxide-based systems such as $\mathrm{ZnO}, \mathrm{Au} @ \mathrm{ZnO}, \mathrm{HfO}_{2}{ }^{8.4 \mathrm{~nm}}, \mathrm{HfO}_{2}{ }^{75} \mathrm{~nm}$, and $\mathrm{Au} @ \mathrm{HfO}_{2}{ }^{75 \mathrm{~nm}}$. High radiosensitization was attained with $\mathrm{Au}$ supported on $\mathrm{TiO}_{2}{ }^{21.6 \mathrm{~nm}}$ and $\mathrm{HfO}_{2}{ }^{8.4}$ ${ }^{n m}$ with degradation enhancements of $57.1 \%$ and $42.3 \%$ respectively. These results corroborate the high physical enhancement of nanomaterials composed of high Z elements such as Au and Hf. 
Radiosensitization of $\mathrm{Au} @ \mathrm{TiO}_{2}$ was also tested in vitro experiments using M. aeruginosa cells. SEM images showed that the combined effect of the nanocomposites and X-rays produces rupture on the mucilaginous outer layer of the cells due to the attack of the ROS created by the $\mathrm{Au} @ \mathrm{TiO}_{2}$ nanocomposites. The radiation enhancement of both $\mathrm{TiO}_{2}$ and $\mathrm{Au} @ \mathrm{TiO}_{2}$ was tested by monitoring the cell density using UV-Vis, and results showed a cell population decrease of $26.8 \%$ and $38.6 \%$ when the particles were irradiated in the presence of $\mathrm{TiO}_{2}$ and $\mathrm{Au} @ \mathrm{TiO}_{2}$ respectively. One of the most interesting findings in this work is the anti-enhancement phenomena that $\mathrm{TiO}_{2}$ showed in the experiments carried out using MB as a chemical dosimeter. Nonetheless, a significant biological enhancement was detected in $\mathrm{M}$. aeruginosa cells irradiated in the presence of $\mathrm{TiO}_{2}$. Further investigation needs to be done on bare $\mathrm{TiO}_{2}$ nanoparticles in order to increase the physical and chemical enhancement of support. This could potentially improve the radiosensitization of $\mathrm{Au} @ \mathrm{TiO}_{2}$ nanocomposites.

$\mathrm{Au} @ \mathrm{TiO}_{2}$ nanocomposites were synthesized using X-ray radiolytic synthesis, a compelling fabrication method with the capability of producing nanomaterials in absence of complex environments of high temperature or pressure, and without the need for toxic chemical reducing agents. $\mathrm{Au} @ \mathrm{TiO}_{2}$ nanocomposites were synthesized through X-ray radiolysis, using different ligands such as $\mathrm{NaOH}$ and Urea and total absorbed doses from 120 to $7260 \mathrm{~Gy}$. Results showed that an increment on the absorbed dose leads to the production of a higher number of reducing species such as $\mathrm{e}^{-}$aq and $\mathrm{H}^{\bullet}$ that create a high number of seeds leading to small nanoparticles homogenously distributed on the support surface. The mean Au particle size changed from $5.7 \pm 1.5 \mathrm{~nm}$ to $1.2 \pm$ $0.2 \mathrm{~nm}$ when the absorbed dose was varied from $120 \mathrm{~Gy}$ to $7260 \mathrm{~Gy}$. Moreover, X-ray radiolytic synthesis of $\mathrm{Au} @ \mathrm{TiO}_{2}$ nanocomposites shows an improvement in the loading and the monodispersity of the supported $\mathrm{Au}$ nanoparticles when compared to traditional chemical 
approaches such as deposition-precipitation. Synthesis of $\mathrm{Au} @ \mathrm{TiO}_{2}$ was carried out using $\mathrm{NaOH}$ and Urea as a ligand, resulting in Au nanoparticles with a mean particle size of $\sim 1.0 \mathrm{~nm}$ and narrow particle size distribution, when high absorbed doses such as 720 and $7260 \mathrm{~Gy}$ are used. Nonetheless, radiosensitization analysis of $\mathrm{Au} @ \mathrm{TiO}_{2}, \mathrm{Au} @ \mathrm{ZnO}$, and $\mathrm{Au} @ \mathrm{HfO}_{2}{ }^{75 m}$ synthesized with urea as la igand, showed low radiation enhancement, due to the urea residues on the particle surface as detected by XPS and TGA. Furthermore, analysis on the particle surface made by XPS showed that Au bond upon the surface of $\mathrm{TiO}_{2}$ through Au-O bonds with a binding energy of $532.0 \mathrm{eV}$. This suggest that $\mathrm{Au}$ species interact with the $\mathrm{OH}$ groups promoted by the ligands on the surface of the $\mathrm{TiO}_{2}$. This work shows that $\mathrm{X}$-ray radiolytic synthesis is a suitable technique to synthesize nanocomposites that could be used in several medical and catalytic applications.

\subsection{Future work.}

Future work on the radiosensitization of $\mathrm{Au} @ \mathrm{TiO}_{2}$ will be based on i) Quantification of ROS induced by oxides and Au@oxides ii) Comparison of radiosensitization efficiency with photodynamic therapy iii). Scaling up X-ray radiolytic synthesis technology for industrial applications. In order to answer these questions, the radiosensitization of oxides and Au@oxides will be tested by monitoring the degradation of different dyes in the presence of the nanoparticles, as a function of parameters such as oxide particle size, $\mathrm{OH} \bullet$ and superoxide radical production, and photon energy. Moreover, the biological response of $\mathrm{Au} @ \mathrm{TiO}_{2}$ and other $\mathrm{Au} @$ oxides needs to be tested in human cells in order to measure the efficiency of ROS in DNA and malignant tissue damage. One of the limitations that our project has is related to the uncertainty that nanomaterials face in the field of medicine regarding their cytotoxicity, cellular stress and circulation time in the human body. Therefore, in-vitro studies that focus on the evaluation of these parameters need to be made in order to enable the use of $\mathrm{Au} @ \mathrm{TiO}_{2}$ in the nanomedicine field. In order for $\mathrm{Au} @ \mathrm{TiO}_{2}$ 
nanocomposites to be used, studies regarding nanoparticle behavior in different organs, cells and patients will facilitate regulations that could potentially enable the use of nanomaterials as therapeutic agents commercially. Furthermore, the radiation enhancement results presented in this project do not account for the radiation attenuation caused by the human body, this could possibly lead to different values of radiation enhancement when the nanocomposites are tested using in-vitro techniques. Currently, our group is studying the possibility of fluorescent bioconjugation of $\mathrm{Au} @ \mathrm{TiO}_{2}$ nanocomposites for future in-vivo and in-vitro testing. For this purpose, $\mathrm{TiO}_{2}$ and $\mathrm{Au} @ \mathrm{TiO}_{2}$ are being functionalized and conjugated to targeting moieties aiming at delivering the material to the target cells.

The morphology and structure of the support is an important feature of the nanocomposite, therefore studies on supports with different morphology need to be made. Supports can be of a porous nature, such as zeolites or mesoporous structures. The use of porous materials in catalysis allows for the creation of many adsorption sites for nanoparticles to grow [159]. Moreover, deposition of metallic nanoparticles onto porous supports leads to small particles with low aggregation due to a limited particle growth caused by the physical morphology of the support [159]. Moreover, the morphology of the pores can affect the resulting size and shape of the supported nanoparticle. An example of a porous material used as a support is silica mesoporous nanomaterials, where doping of other metals such as Al, Ti, and Ce within the silica framework increased their overall chemical reactivity towards a variety of catalytic reactions [159]. Carbon supports with well-defined porosities have been also explored within the field as supports of nanomaterials. The topography of the supports can be controlled and modified through techniques such as ozonolysis, plasma, doping, and acidic treatments, in order to increase the number of adsorption sites for nanoparticles to nucleate and grow 
[159]. Carbon nanotubes are one of the most promising supports for metal nanoparticles due to their high surface area, high electrical conductivity, small size and hollow geometry [159].

Radiosensitization of $\mathrm{Au} @ \mathrm{TiO}_{2}$ has been tested by activating the particles with X-rays, using energies in the kilovoltage and megavoltage range in a MB solution, where the absorption of X-rays by the particles leads to the emission of photoelectrons, Auger electrons, characteristic X-rays and ROS, species that contribute to increasing dose rate in a concept that could be used to enhance dye degradation in water streams. One of the most challenging problem in the catalysis field to improve water quality by removing efficiently contaminates coming from different sources such as dyes, household chemicals and pesticides [160]. Due to the complexity of the many forms of pollution, there is a strong need to monitor and control emerging pollutants and create new wastewater treatment strategies that mitigate the negative effects of polluted water. Textile dyes are the second largest pollutant of clean water globally, after agriculture [161]. The release of these dyes to the freshwater effluents has become an important source of environmental contamination. Thus, the study of advanced wastewater treatments could mitigate some of the consequences of dye polluted water in aquatic ecosystems. Some of the negative effects of polluted water in the environment include sunlight penetration reduction, toxic, mutagenic and carcinogenic effects on the aquatic ecosystem [17]. Moreover, dye-contaminated water can cause abdominal discomfort and general irritation in the digestive system in human populations that rely on water for drinking and cooking [17]. The combination of a metal oxide semiconductors and ionizing radiation for water treatment is a strategy recently explored in a process known as radiocatalysis $[147,162]$. The catalytic properties of metal oxide semiconductors and penetrating radiation could provide a faster degradation rate of pollutants than conventional methods such as photocatalysis [17]. 
A systematic study regarding different metal nanoparticles onto $\mathrm{TiO}_{2}$ needs to be made, in order to evaluate the best nanocomposite for dye degradation purposes in the presence of ionizing radiation. Metals such as $\mathrm{Cu}, \mathrm{Au}$, and $\mathrm{Ag}$ have been deposited onto titania films and results showed that $\mathrm{Au}$ and $\mathrm{Cu}$ nanoparticles had a higher photocatalytic performance than silver. The superior enhancement was found due to the capability of $\mathrm{Au}$ and $\mathrm{Cu}$ electron capture, delaying the recombination rate of electron-holes produced by $\mathrm{TiO}_{2}$ [163]. Another parameter that could affect pollutant decomposition is the shape of the supported nanoparticle, Au particles with different shapes such as spheres, rods, and cubes were deposited onto commercial titania. The electronic state of the titania was evaluated using attenuated total reflectance (ATR) in the presence of Au nanorods with a fixed diameter of $25 \mathrm{~nm}$ and different lengths. Results showed that there was not significant change in the ATR spectra, therefore changes in the electronic state of $\mathrm{TiO}_{2}$ depend on the Au diameter rather than the length of the nanorods [164]. Furthermore, deposition of gold nanospheres on $\mathrm{TiO}_{2}$ with a mean particle size of 5, 10, 20 and $60 \mathrm{~nm}$ was examined. Results showed that the photocatalytic activity increased with a decreased particle size and ATR showed a large electronic state change, which led to a higher charge-separation efficiencies. Therefore, this work concluded that the electronic states of $\mathrm{TiO}_{2}$ are dependent on the Au size rather than Au shape [164].

In order to test the potential uses of $\mathrm{Au} @ \mathrm{TiO}_{2}$ as radiocatalysts, the recoverability and reusability of $\mathrm{Au} @ \mathrm{TiO}_{2}$ nanocomposites for environmental remediation is shown in Figure 8.1. For this purpose, $\mathrm{Au} @ \mathrm{TiO}_{2}$ was added to $\mathrm{MB}$ and irradiated for 8 minutes at a dose rate of $35 \mathrm{~Gy}$ using an operating voltage of $50 \mathrm{kV}$. After irradiation, the solution was centrifuged, the nanomaterial recovered, and $\mathrm{Au} @ \mathrm{TiO}_{2}$ nanocomposites were thoroughly washed with water, in order to remove dye residues from its surface. This process was repeated 10 consecutive cycles. Results showed that after the fourth cycle, the nanomaterial decreases its performance. Nonetheless, after 10 cycles of irradiation, 
$\mathrm{Au} @ \mathrm{TiO}_{2}$ nanocomposites showed a superior $\mathrm{MB}$ degradation rate enhancement of $43.6 \%$ when compared with the control. The results indicate $\mathrm{Au} @ \mathrm{TiO}_{2}$ recoverability and their potential use as a catalyst for dye degradation throughout several cycles.

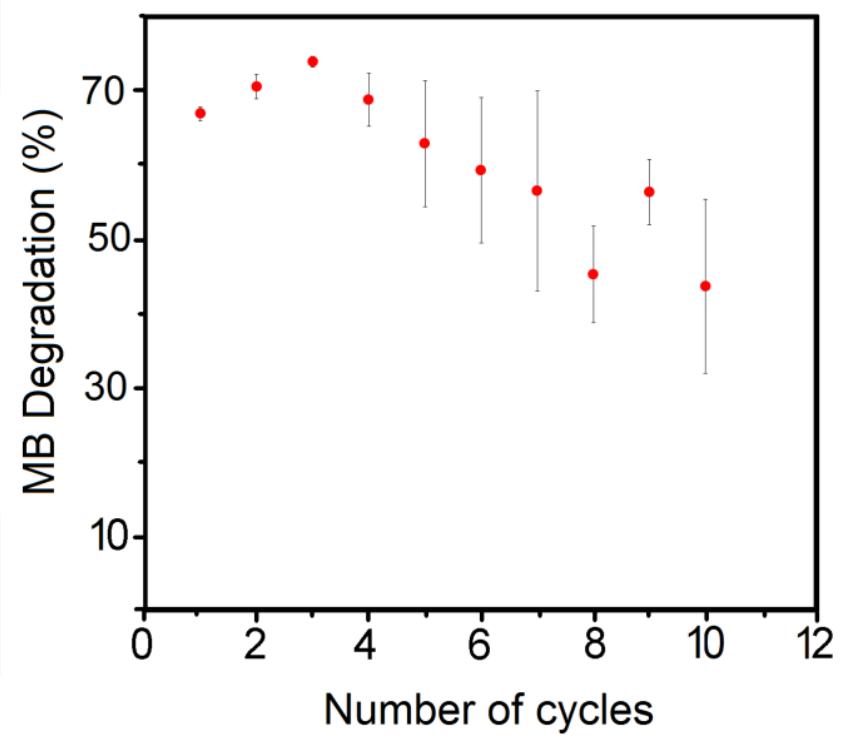

Figure 8.1. Degradation of MB after 10 cycles using $\mathrm{Au} @ \mathrm{TiO}_{2}{ }^{(6.5 \mathrm{~nm})-\mathrm{NaOH}}$ nanocomposites. Irradiation carried out at $50 \mathrm{KV}$ and $35 \mathrm{~Gy} / \mathrm{min}$ 


\section{List of References}

[1] G. Delaney, S. Jacob, C. Featherstone, M. Barton, The role of radiotherapy in cancer treatment: estimating optimal utilization from a review of evidence-based clinical guidelines, Cancer. 104 (2005) 1129-1137. doi:10.1002/cncr.21324.

[2] S.M. Bentzen, Preventing or reducing late side effects of radiation therapy: radiobiology meets molecular pathology, Nat. Rev. Cancer. 6 (2006) 702-713. doi:10.1038/nrc1950.

[3] S. Her, D.A. Jaffray, C. Allen, Gold nanoparticles for applications in cancer radiotherapy: Mechanisms and recent advancements, Adv. Drug Deliv. Rev. 109 (2017) 84-101. doi:10.1016/j.addr.2015.12.012.

[4] Deep Kwatra, A. Venugopal, S. Anant, Nanoparticles in radiation therapy: a summary of various approaches to enhance radiosensitization in cancer, Transl Cancer Res. 2 (2013).

[5] H. Wang, X. Mu, H. He, X.-D. Zhang, Cancer Radiosensitizers, Trends Pharmacol. Sci. 39 (2018) 24-48. doi:10.1016/j.tips.2017.11.003.

[6] Y. Liu, P. Zhang, F. Li, X. Jin, J. Li, W. Chen, Q. Li, Metal-based NanoEnhancers for Future Radiotherapy: Radiosensitizing and Synergistic Effects on Tumor Cells, Theranostics. 8 (2018) 1824-1849. doi:10.7150/thno.22172.

[7] X.-Y. Su, P.-D. Liu, H. Wu, N. Gu, Enhancement of radiosensitization by metal-based nanoparticles in cancer radiation therapy, Cancer Biol. Med. 11 (2014) 86-91. doi:10.7497/j.issn.2095-3941.2014.02.003.

[8] S. Klein, M.L. Dell'Arciprete, M. Wegmann, L.V.R. Distel, W. Neuhuber, M.C. Gonzalez, C. Kryschi, Oxidized silicon nanoparticles for radiosensitization of cancer and tissue cells, Biochem. Biophys. Res. Commun. 434 (2013) 217-222. doi:10.1016/j.bbrc.2013.03.042. 
[9] E.Q. Youkhana, B. Feltis, A. Blencowe, M. Geso, Titanium Dioxide Nanoparticles as Radiosensitisers: An In vitro and Phantom-Based Study, Int. J. Med. Sci. 14 (2017) 602-614. doi:10.7150/ijms.19058.

[10] T.L. McGinnity, O. Dominguez, T.E. Curtis, P.D. Nallathamby, A.J. Hoffman, R.K. Roeder, Hafnia (HfO2) nanoparticles as an X-ray contrast agent and mid-infrared biosensor, Nanoscale. 8 (2016) 13627-13637. doi:10.1039/C6NR03217F.

[11] R. Generalov, W.B. Kuan, W. Chen, S. Kristensen, P. Juzenas, Radiosensitizing effect of zinc oxide and silica nanocomposites on cancer cells, Colloids Surf. B Biointerfaces. 129 (2015) 79-86. doi:10.1016/j.colsurfb.2015.03.026.

[12] P. Juzenas, W. Chen, Y.-P. Sun, M.A.N. Coelho, R. Generalov, N. Generalova, I.L. Christensen, Quantum dots and nanoparticles for photodynamic and radiation therapies of cancer, Adv. Drug Deliv. Rev. 60 (2008) 1600-1614. doi:10.1016/j.addr.2008.08.004.

[13] A. Mesbahi, A review on gold nanoparticles radiosensitization effect in radiation therapy of cancer, Rep. Pract. Oncol. Radiother. 15 (2010) 176-180. doi:10.1016/j.rpor.2010.09.001.

[14] Y. Dou, Y. Guo, X. Li, X. Li, S. Wang, L. Wang, G. Lv, X. Zhang, H. Wang, X. Gong, J. Chang, Size-Tuning Ionization To Optimize Gold Nanoparticles for Simultaneous Enhanced CT Imaging and Radiotherapy, ACS Nano. 10 (2016) 2536-2548. doi:10.1021/acsnano.5b07473.

[15] E. Brun, L. Sanche, C. Sicard-Roselli, Parameters governing gold nanoparticle X-ray radiosensitization of DNA in solution, Colloids Surf. B Biointerfaces. 72 (2009) 128-134. doi:10.1016/j.colsurfb.2009.03.025.

[16] X. Yao, C. Huang, X. Chen, Z. Yi, L. Sanche, Chemical Radiosensitivity of DNA Induced by Gold Nanoparticles, J. Biomed. Nanotechnol. 11 (2015) 478-485. 
[17] M. Molina Higgins, M. Toro González, J. Rojas, Enhanced X-RAYS degradation of methylene blue in the presence of gold microspheres, Radiat. Phys. Chem. 156 (2019) 7380. doi:10.1016/j.radphyschem.2018.10.020.

[18] E. Lechtman, N. Chattopadhyay, Z. Cai, S. Mashouf, R. Reilly, J.P. Pignol, Implications on clinical scenario of gold nanoparticle radiosensitization in regards to photon energy, nanoparticle size, concentration and location, Phys. Med. Biol. 56 (2011) 4631-4647. doi:10.1088/0031-9155/56/15/001.

[19] D.M. Herold, I.J. Das, C.C. Stobbe, R.V. Iyer, J.D. Chapman, Gold microspheres: a selective technique for producing biologically effective dose enhancement, Int. J. Radiat. Biol. 76 (2000) 1357-1364.

[20] Z. Kuncic, S. Lacombe, Nanoparticle radio-enhancement: principles, progress and application to cancer treatment, Phys. Med. Biol. 63 (2018) 02TR01. doi:10.1088/13616560/aa99ce.

[21] G. Prieto, J. Zečević, H. Friedrich, K.P. de Jong, P.E. de Jongh, Towards stable catalysts by controlling collective properties of supported metal nanoparticles, Nat. Mater. 12 (2013) 3439. doi:10.1038/nmat3471.

[22] Z.F. Yin, L. Wu, H.G. Yang, Y.H. Su, Recent progress in biomedical applications of titanium dioxide, Phys. Chem. Chem. Phys. 15 (2013) 4844-4858. doi:10.1039/C3CP43938K.

[23] N. Kotagiri, G.P. Sudlow, W.J. Akers, S. Achilefu, Breaking the Depth Dependency of Phototherapy with Cerenkov Radiation and Low Radiance Responsive Nanophotosensitizers, Nat. Nanotechnol. 10 (2015) 370-379. doi:10.1038/nnano.2015.17. 
[24] J.A. Reyes-Esqueda, A.B. Salvador, R. Zanella, Size control of Au nanoparticles on TiO2 and $\mathrm{Al} 2 \mathrm{O} 3$ by DP Urea: optical absorption and electron microscopy as control probes, J. Nanosci. Nanotechnol. 8 (2008) 3843-3850.

[25] Q. Yao, C. Wang, H. Wang, H. Yan, J. Lu, Revisiting the Au Particle Size Effect on TiO2Coated Au/TiO2 Catalysts in CO Oxidation Reaction, J. Phys. Chem. C. 120 (2016) 91749183. doi:10.1021/acs.jpcc.5b12712.

[26] M. Du, D. Sun, H. Yang, J. Huang, X. Jing, T. Odoom-Wubah, H. Wang, L. Jia, Q. Li, Influence of Au Particle Size on Au/TiO2 Catalysts for CO Oxidation, J. Phys. Chem. C. 118 (2014) 19150-19157. doi:10.1021/jp504681f.

[27] G. Sharma, D. Kumar, A. Kumar, A.H. Al-Muhtaseb, D. Pathania, M. Naushad, G.T. Mola, Revolution from monometallic to trimetallic nanoparticle composites, various synthesis methods and their applications: A review, Mater. Sci. Eng. C Mater. Biol. Appl. 71 (2017) 1216-1230. doi:10.1016/j.msec.2016.11.002.

[28] X.Y. Liu, J.H. Peng, Y.X. Zhang, One-pot synthesis for Lysie-capped Au-TiO2 binary nanocomposites, Ceram. Int. 42 (2016) 19450-19453. doi:10.1016/j.ceramint.2016.09.103.

[29] R. Zanella, S. Giorgio, C.R. Henry, C. Louis, Alternative Methods for the Preparation of Gold Nanoparticles Supported on TiO2, J. Phys. Chem. B. 106 (2002) 7634-7642. doi:10.1021/jp0144810.

[30] J. Belloni, M. Mostafavi, H. Remita, J.-L. Marignier, and M.-O. Delcourt, Radiation-induced synthesis of mono- and multi-metallic clusters and nanocolloids, New J. Chem. 22 (1998) 1239-1255. doi:10.1039/A801445K. 
[31] S. Anandan, M. Ashokkumar, Sonochemical synthesis of Au-TiO2 nanoparticles for the sonophotocatalytic degradation of organic pollutants in aqueous environment, Ultrason. Sonochem. 16 (2009) 316-320. doi:10.1016/j.ultsonch.2008.10.010.

[32] S.-H. Baeck, T.F. Jaramillo, A. Kleiman-Shwarsctein, E.W. McFarland, Automated electrochemical synthesis and characterization of $\mathrm{TiO} 2$ supported $\mathrm{Au}$ nanoparticle electrocatalysts, Meas. Sci. Technol. 16 (2005) 54. doi:10.1088/0957-0233/16/1/008.

[33] F. Moslehirad, M.H.M. Ara, M.J. Torkamany, Synthesis of composite Au/TiO 2 nanoparticles through pulsed laser ablation and study of their optical properties, Laser Phys. 23 (2013) 075601. doi:10.1088/1054-660X/23/7/075601.

[34] M. Rohe, E. Löffler, M. Muhler, A. Birkner, C. Wöll, K. Merz, A gold-containing TiO complex: a crystalline molecular precursor as an alternative route to $\mathrm{Au} / \mathrm{TiO} 2$ composites, Dalton Trans. Camb. Engl. 2003. (2008) 6106-6109. doi:10.1039/b810929j.

[35] D.M. Clifford, C.E. Castano, J.V. Rojas, Supported transition metal nanomaterials: Nanocomposites synthesized by ionizing radiation, Radiat. Phys. Chem. 132 (2017) 52-64. doi:10.1016/j.radphyschem.2016.12.001.

[36] O.V. Kharissova, B.I. Kharisov, U.O. Mndez, Radiation-Assisted Synthesis of Composites, Materials, Compounds, and Nanostructures, in: Wiley Encycl. Compos., American Cancer Society, 2012: pp. 1-26. doi:10.1002/9781118097298.weoc212.

[37] J.V. Rojas, M. Toro-Gonzalez, M.C. Molina-Higgins, C.E. Castano, Facile radiolytic synthesis of ruthenium nanoparticles on graphene oxide and carbon nanotubes, Mater. Sci. Eng. B. 205 (2016) 28-35. doi:10.1016/j.mseb.2015.12.005. 
[38] J.V. Rojas, M.C. Molina Higgins, M. Toro Gonzalez, C.E. Castano, Single step radiolytic synthesis of iridium nanoparticles onto graphene oxide, Appl. Surf. Sci. 357 (2015) 20872093. doi:10.1016/j.apsusc.2015.09.190.

[39] M.C. Molina Higgins, D.M. Clifford, J.V. Rojas, Au@TiO2 nanocomposites synthesized by X-ray radiolysis as potential radiosensitizers, Appl. Surf. Sci. 427 (2018) 702-710. doi:10.1016/j.apsusc.2017.08.094.

[40] A. Bharti, R. Bhardwaj, A.K. Agrawal, N. Goyal, S. Gautam, Monochromatic X-Ray Induced Novel Synthesis of Plasmonic Nanostructure for Photovoltaic Application, Sci. Rep. 6 (2016) 22394. doi:10.1038/srep22394.

[41] M. Simic, P. Neta, E. Hayon, Pulse radiolysis study of alcohols in aqueous solution, J. Phys. Chem. 73 (1969) 3794-3800. doi:10.1021/j100845a038.

[42] H. Remita, I. Lampre, M. Mostafavi, E. Balanzat, S. Bouffard, Comparative study of metal clusters induced in aqueous solutions by $\gamma$-rays, electron or $\mathrm{C} 6+$ ion beam irradiation, Radiat. Phys. Chem. 72 (2005) 575-586. doi:10.1016/j.radphyschem.2004.03.042.

[43] N.D. Nguyen, V.P. Dang, A.Q. Le, Q.H. Nguyen, Electron beam/ $\gamma$-ray irradiation synthesis of gold nanoparticles and investigation of antioxidant activity, Adv. Nat. Sci. Nanosci. Nanotechnol. 5 (2014) 045002. doi:10.1088/2043-6262/5/4/045002.

[44] J.V. Rojas, C.H. Castano, Radiation-assisted synthesis of iridium and rhodium nanoparticles supported on polyvinylpyrrolidone, J. Radioanal. Nucl. Chem. 302 (2014) 555-561. doi:10.1007/s10967-014-3291-y.

[45] S. Remita, P. Fontaine, E. Lacaze, Y. Borensztein, H. Sellame, R. Farha, C. Rochas, M. Goldmann, X-ray radiolysis induced formation of silver nano-particles: A SAXS and UV- 
visible absorption spectroscopy study, Nucl. Instrum. Methods Phys. Res. Sect. B Beam Interact. Mater. At. 263 (2007) 436-440. doi:10.1016/j.nimb.2007.06.032.

[46] R. Zanella, L. Delannoy, C. Louis, Mechanism of deposition of gold precursors onto TiO2 during the preparation by cation adsorption and deposition-precipitation with $\mathrm{NaOH}$ and urea, Appl. Catal. Gen. 291 (2005) 62-72. doi:10.1016/j.apcata.2005.02.045.

[47] B. Zheng, Z. Zheng, J. Zhang, X. Luo, Q. Liu, J. Wang, Y. Zhao, The removal of Microcystis aeruginosa in water by gamma-ray irradiation, Sep. Purif. Technol. 85 (2012) 165-170. doi:10.1016/j.seppur.2011.10.005.

[48] C.H. Ruhlmann, T.Z. Iversen, M. Okera, A. Muhic, G. Kristensen, P. Feyer, O. Hansen, J. Herrstedt, Multinational study exploring patients' perceptions of side-effects induced by chemo-radiotherapy, Radiother. Oncol. J. Eur. Soc. Ther. Radiol. Oncol. 117 (2015) 333337. doi:10.1016/j.radonc.2015.09.014.

[49] Z. Hai, N.E. Kolli, J. Chen, H. Remita, Radiolytic synthesis of $\mathrm{Au}-\mathrm{Cu}$ bimetallic nanoparticles supported on TiO2: application in photocatalysis, New J. Chem. 38 (2014) 5279-5286. doi:10.1039/C4NJ00883A.

[50] J.F. Dorsey, L. Sun, D.Y. Joh, A. Witztum, G.D. Kao, M. Alonso-Basanta, S. Avery, S.M. Hahn, A. Al Zaki, A. Tsourkas, Gold nanoparticles in radiation research: potential applications for imaging and radiosensitization, Transl. Cancer Res. 2 (2013) 280-291. doi:10.3978/j.issn.2218-676X.2013.08.09.

[51] N.T.K. Thanh, N. Maclean, S. Mahiddine, Mechanisms of Nucleation and Growth of Nanoparticles in Solution, Chem. Rev. 114 (2014) 7610-7630. doi:10.1021/cr400544s. 
[52] J. Belloni, Nucleation, growth and properties of nanoclusters studied by radiation chemistry: Application to catalysis, Catal. Today. 113 (2006) 141-156. doi:10.1016/j.cattod.2005.11.082.

[53] A. Abedini, A.R. Daud, M.A. Abdul Hamid, N. Kamil Othman, E. Saion, A review on radiation-induced nucleation and growth of colloidal metallic nanoparticles, Nanoscale Res. Lett. 8 (2013) 474. doi:10.1186/1556-276X-8-474.

[54] C.-J. Liu, T.-Y. Yang, C.-H. Wang, C.-C. Chien, S.-T. Chen, C.-L. Wang, W.-H. Leng, Y. Hwu, H.-M. Lin, Y.-C. Lee, C.-L. Cheng, J.H. Je, G. Margaritondo, Enhanced photocatalysis, colloidal stability and cytotoxicity of synchrotron X-ray synthesized Au/TiO2 nanoparticles, Mater. Chem. Phys. 117 (2009) 74-79. doi:10.1016/j.matchemphys.2009.05.030.

[55] P. Hoskin, Basic Physics, in: Extern. Beam Ther., Oxford University Press, 2012: pp. 6-25.

[56] L.K. Schubert, M. Miften, The Physics of Brachytherapy, in: P. Montemaggi, M. Trombetta, L.W. Brady (Eds.), Brachytherapy Int. Perspect., Springer International Publishing, Cham, 2016: pp. 13-27. doi:10.1007/978-3-319-26791-3_3.

[57] P. Lesueur, V. Calugaru, C. Nauraye, D. Stefan, K. Cao, E. Emery, Y. Reznik, J.L. Habrand, T. Tessonnier, A. Chaikh, J. Balosso, J. Thariat, Proton therapy for treatment of intracranial benign tumors in adults: A systematic review, Cancer Treat. Rev. 72 (2019) 56-64. doi:10.1016/j.ctrv.2018.11.004.

[58] S. Diamantopoulos, K. Platoni, M. Dilvoi, I. Nazos, K. Geropantas, G. Maravelis, M. Tolia, I. Beli, E. Efstathopoulos, P. Pantelakos, G. Panayiotakis, V. Kouloulias, Clinical implementation of total skin electron beam (TSEB) therapy: A review of the relevant literature, Phys. Med. 27 (2011) 62-68. doi:10.1016/j.ejmp.2010.09.001. 
[59] N.P. Rowell, C.J. Williams, Radical radiotherapy for stage I/II non-small cell lung cancer in patients not sufficiently fit for or declining surgery (medically inoperable), Cochrane Database Syst. Rev. (2001) CD002935. doi:10.1002/14651858.CD002935.

[60] D.A. Jaffray, M.K. Gospodarowicz, Radiation Therapy for Cancer, in: Cancer Dis. Control Priorities Third Ed. Vol. 3, The International Bank for Reconstruction and Development / The World Bank, Washington (DC), 2015.

[61] M.R. Bonomi, A. Blakaj, D. Blakaj, Organ preservation for advanced larynx cancer: A review of chemotherapy and radiation combination strategies, Oral Oncol. 86 (2018) 301-306. doi:10.1016/j.oraloncology.2018.10.004.

[62] O. Ung, A.O. Langlands, B. Barraclough, J. Boyages, Combined chemotherapy and radiotherapy for patients with breast cancer and extensive nodal involvement, J. Clin. Oncol. Off. J. Am. Soc. Clin. Oncol. 13 (1995) 435-443. doi:10.1200/JCO.1995.13.2.435.

[63] A. Chan, A. Wong, Is combined chemotherapy and radiation therapy equally effective as surgical resection in localized esophageal carcinoma?, Int. J. Radiat. Oncol. Biol. Phys. 45 (1999) 265-270.

[64] B. van Oorschot, D. Rades, W. Schulze, G. Beckmann, P. Feyer, Palliative radiotherapy-new approaches, Semin. Oncol. 38 (2011) 443-449. doi:10.1053/j.seminoncol.2011.03.015.

[65] S.T. Lutz, J. Jones, E. Chow, Role of Radiation Therapy in Palliative Care of the Patient With Cancer, J. Clin. Oncol. 32 (2014) 2913-2919. doi:10.1200/JCO.2014.55.1143.

[66] O. Desouky, N. Ding, G. Zhou, Targeted and non-targeted effects of ionizing radiation, J. Radiat. Res. Appl. Sci. 8 (2015) 247-254. doi:10.1016/j.jrras.2015.03.003.

[67] R. Baskar, K.A. Lee, R. Yeo, K.-W. Yeoh, Cancer and Radiation Therapy: Current Advances and Future Directions, Int. J. Med. Sci. 9 (2012) 193-199. doi:10.7150/ijms.3635. 
[68] S. Mehta, V. Suhag, M. Semwal, N. Sharma, Radiotherapy: Basic Concepts and Recent Advances, Med. J. Armed Forces India. 66 (2010) 158-162. doi:10.1016/S03771237(10)80132-7.

[69] G. Hajdok, J. Yao, J.J. Battista, I.A. Cunningham, Signal and noise transfer properties of photoelectric interactions in diagnostic x-ray imaging detectors, Med. Phys. 33 (2006) 36013620. doi:10.1118/1.2336507.

[70] B.L. Hoffman, J.O. Schorge, J.I. Schaffer, L.M. Halvorson, K.D. Bradshaw, F.G. Cunningham, L.E. Calver, Principles of Radiation Therapy, in: Williams Gynecol., 2nd ed., The McGraw-Hill Companies, New York, NY, 2012.

[71] L. Best, G. Rodrigues, V. Velker, Basic science concepts, in: Radiat. Oncol. Primer Rev. Essent. Concepts Protoc., Demos Medical Publishing, 2013: pp. 48-49.

[72] S. Nill, T. Tücking, M.W. Münter, U. Oelfke, Intensity modulated radiation therapy with multileaf collimators of different leaf widths: a comparison of achievable dose distributions, Radiother. Oncol. J. Eur. Soc. Ther. Radiol. Oncol. 75 (2005) 106-111. doi:10.1016/j.radonc.2005.02.007.

[73] B. Cho, Intensity-modulated radiation therapy: a review with a physics perspective, Radiat. Oncol. J. 36 (2018) 1-10. doi:10.3857/roj.2018.00122.

[74] E.B. Podgoršak, J.A. Rawlinson, M.I. Glavinović, H.E. Johns, Design of x-ray targets for high energy linear accelerators in radiotherapy, Am. J. Roentgenol. 121 (1974) 873-882. doi:10.2214/ajr.121.4.873.

[75] C.P. Joshi, S. Dhanesar, J. Darko, A. Kerr, P.B. Vidyasagar, L.J. Schreiner, Practical and clinical considerations in Cobalt-60 tomotherapy, J. Med. Phys. Assoc. Med. Phys. India. 34 (2009) 137-140. doi:10.4103/0971-6203.54847. 
[76] E. Delikgoz Soykut, E.M. Ozsahin, Y. Yukselen Guney, S. Aytac Arslan, O. Derinalp Or, M.B. Altundag, G. Ugurluer, P.G. Tsoutsou, The use of PET/CT in radiotherapy planning: contribution of deformable registration, Front. Oncol. 3 (2013). doi:10.3389/fonc.2013.00033.

[77] C.A. Kelsey, P.H. Heintz, D.J. Sandoval, G.D. Chambers, N.L. Adolphi, K.S. Paffett, eds., Radiation Treatment of Cancer, in: Radiat. Biol. Med. Imaging, John Wiley \& Sons, Inc., Hoboken, NJ, USA, 2014: pp. 191-202. doi:10.1002/9781118517154.ch11.

[78] RCR Radiotherapy Dose Fractionation Second Edition (UK), Xstrahl Med. Life Sci. (n.d.). https://xstrahl.com/medical-publication/rcr-radiotherapy-dose-fractionation-second-editionuk/ (accessed January 30, 2019).

[79] G. Clark, Radiation Therapy Fractionation and Dosing Schemes, in: Oklahoma, 2012: p. 62.

[80] K. Al-Saleh, R. Safwat, H.S. Hooda, A. Bedair, A. Al-Basmy, H. Al-Wikeel, M. Naseer, Z. Thotathil, Hyperfractionated radiation therapy and concurrent chemotherapy for advanced head and neck cancer, Gulf J. Oncolog. (2011) 12-19.

[81] S.C. King, J.C. Acker, P.S. Kussin, L.B. Marks, K.J. Weeks, K.A. Leopold, High-dose, hyperfractionated, accelerated radiotherapy using a concurrent boost for the treatment of nonsmall cell lung cancer: unusual toxicity and promising early results, Int. J. Radiat. Oncol. Biol. Phys. 36 (1996) 593-599.

[82] T.W. Griffin, Fast neutron radiation therapy, Crit. Rev. Oncol. Hematol. 13 (1992) 17-31.

[83] C.M. Ma, T. Pawlicki, M.C. Lee, S.B. Jiang, J.S. Li, J. Deng, B. Yi, E. Mok, A.L. Boyer, Energy- and intensity-modulated electron beams for radiotherapy, Phys. Med. Biol. 45 (2000) 2293-2311. 
[84] H. Tsujii, J.-E. Mizoe, T. Kamada, M. Baba, S. Kato, H. Kato, H. Tsuji, S. Yamada, S. Yasuda, T. Ohno, T. Yanagi, A. Hasegawa, T. Sugawara, H. Ezawa, S. Kandatsu, K. Yoshikawa, R. Kishimoto, T. Miyamoto, Overview of clinical experiences on carbon ion radiotherapy at NIRS, Radiother. Oncol. J. Eur. Soc. Ther. Radiol. Oncol. 73 Suppl 2 (2004) S41-49.

[85] J.K. Rockhill, G.E. Laramore, Neutron Radiotherapy, in: Clin. Radiat. Oncol., 4th ed., Elsevier, Philadelphia, PA, 2016: pp. 373-380.e2.

[86] R. Srivastava, B. Jyoti, J. Dixit, P. Priyadarshi, Neutron Therapy-A Novel Approach To Radiotherapeutics: A Review, Int. J. Contemp. Med. Res. 1 (n.d.) 61-70.

[87] IAEA, Current Status of Neutron Capture Therapy, (2001). http://wwwpub.iaea.org/books/IAEABooks/6168/Current-Status-of-Neutron-Capture-Therapy (accessed January 31, 2019).

[88] H. Liu, J.Y. Chang, Proton therapy in clinical practice, Chin. J. Cancer. 30 (2011) 315-326. doi:10.5732/cjc.010.10529.

[89] J. Skowronek, Current status of brachytherapy in cancer treatment - short overview, J. Contemp. Brachytherapy. 9 (2017) 581-589. doi:10.5114/jcb.2017.72607.

[90] F. Ballester, D. Granero, J. Perez-Calatayud, J.L.M. Venselaar, M.J. Rivard, Study of encapsulated sources for their potential use in brachytherapy, Med. Phys. 37 (2010) 16291637. doi:10.1118/1.3360441.

[91] J. Burger, Radioactive sources in brachytherapy, Radiol Oncol. 37 (n.d.) 127-131.

[92] A. Lin, A. Sun, A. Bezjak, J.-P. Bissonnette, J. Tanguay, S. Keshavjee, E.P. Saibishkumar, Lung cancer eight years after radioactive seed migration, Cancer Treat. Res. Commun. 9 (2016) 124-125. doi:10.1016/j.ctarc.2016.09.004. 
[93] S. Gianfaldoni, R. Gianfaldoni, U. Wollina, J. Lotti, G. Tchernev, T. Lotti, An Overview on Radiotherapy: From Its History to Its Current Applications in Dermatology, Open Access Maced. J. Med. Sci. 5 (2017) 521-525. doi:10.3889/oamjms.2017.122.

[94] S.C. Formenti, S. Demaria, Systemic effects of local radiotherapy, Lancet Oncol. 10 (2009) 718-726. doi:10.1016/S1470-2045(09)70082-8.

[95] N. Hamada, Y. Fujimichi, Classification of radiation effects for dose limitation purposes: history, current situation and future prospects, J. Radiat. Res. (Tokyo). 55 (2014) 629-640. doi:10.1093/jrr/rru019.

[96] A. Sourati, A. Ameri, M. Malekzadeh, Acute Side Effects of Radiation Therapy: A Guide to Management, Springer, 2017.

[97] B.T. Oronsky, S.J. Knox, J. Scicinski, Six Degrees of Separation: The Oxygen Effect in the Development of Radiosensitizers, Transl. Oncol. 4 (2011) 189-198.

[98] P. Wardman, Chemical radiosensitizers for use in radiotherapy, Clin. Oncol. R. Coll. Radiol. G. B. 19 (2007) 397-417. doi:10.1016/j.clon.2007.03.010.

[99] S. Rockwell, I.T. Dobrucki, E.Y. Kim, S.T. Marrison, V.T. Vu, Hypoxia and radiation therapy: Past history, ongoing research, and future promise, Curr. Mol. Med. 9 (2009) 442458.

[100] P.J. Hoskin, M.I. Saunders, S. Dische, Hypoxic radiosensitizers in radical radiotherapy for patients with bladder carcinoma: hyperbaric oxygen, misonidazole, and accelerated radiotherapy, carbogen, and nicotinamide, Cancer. 86 (1999) 1322-1328.

[101] G. Thomas, The effect of hemoglobin level on radiotherapy outcomes: the Canadian experience, Semin. Oncol. 28 (2001) 60-65. 
[102] W.A. Denny, P.B. Roberts, R.F. Anderson, J.M. Brown, D. Phil, W.R. Wilson, NLA-1: a 2-nitroimidazole radiosensitizer targeted to DNA by intercalation, Int. J. Radiat. Oncol. Biol. Phys. 22 (1992) 553-556.

[103] M. Bonnet, C.R. Hong, W.W. Wong, L.P. Liew, A. Shome, J. Wang, Y. Gu, R.J. Stevenson, W. Qi, R.F. Anderson, F.B. Pruijn, W.R. Wilson, S.M.F. Jamieson, K.O. Hicks, M.P. Hay, Next-Generation Hypoxic Cell Radiosensitizers: Nitroimidazole Alkylsulfonamides, $\quad$ J. $\quad$ Med. Chem. 61 (2018) 1241-1254. doi:10.1021/acs.jmedchem.7b01678.

[104] S. Liu, H. Li, L. Xia, P. Xu, Y. Ding, D. Huo, Y. Hu, Anti-RhoJ antibody functionalized Au@I nanoparticles as CT-guided tumor vessel-targeting radiosensitizers in patient-derived tumor xenograft model, Biomaterials. $141 \quad$ (2017) 1-12. doi:10.1016/j.biomaterials.2017.06.036.

[105] M. Zheng, S.E. Morgan-Lappe, J. Yang, K.M. Bockbrader, D. Pamarthy, D. Thomas, S.W. Fesik, Y. Sun, Growth inhibition and radiosensitization of glioblastoma and lung cancer cells by small interfering RNA silencing of tumor necrosis factor receptor-associated factor 2 , Cancer Res. 68 (2008) 7570-7578. doi:10.1158/0008-5472.CAN-08-0632.

[106] H. Wang, P. Oliver, Z. Zhang, S. Agrawal, R. Zhang, Chemosensitization and radiosensitization of human cancer by antisense anti-MDM2 oligonucleotides: in vitro and in vivo activities and mechanisms, Ann. N. Y. Acad. Sci. 1002 (2003) 217-235.

[107] U. Kasid, A. Dritschilo, RAF antisense oligonucleotide as a tumor radiosensitizer, Oncogene. 22 (2003) 5876-5884. doi:10.1038/sj.onc.1206700. 
[108] M. Longmire, P.L. Choyke, H. Kobayashi, Clearance Properties of Nano-sized Particles and Molecules as Imaging Agents: Considerations and Caveats, Nanomed. 3 (2008) 703717. doi:10.2217/17435889.3.5.703.

[109] L.X. Pinho, J. Azevedo, Â. Brito, A. Santos, P. Tamagnini, V.J.P. Vilar, V.M. Vasconcelos, R.A.R. Boaventura, Effect of $\mathrm{TiO} 2$ photocatalysis on the destruction of Microcystis aeruginosa cells and degradation of cyanotoxins microcystin-LR and cylindrospermopsin, Chem. Eng. J. 268 (2015) 144-152. doi:10.1016/j.cej.2014.12.111.

[110] J.F. Hainfeld, D.N. Slatkin, H.M. Smilowitz, The use of gold nanoparticles to enhance radiotherapy in mice, Phys. Med. Biol. 49 (2004) N309-315.

[111] C. Suplee, X-Ray Mass Attenuation Coefficients, NIST. (2009). https://www.nist.gov/pml/x-ray-mass-attenuation-coefficients (accessed February 1, 2019).

[112] C. Mirjolet, A.L. Papa, G. Créhange, O. Raguin, C. Seignez, C. Paul, G. Truc, P. Maingon, N. Millot, The radiosensitization effect of titanate nanotubes as a new tool in radiation therapy for glioblastoma: a proof-of-concept, Radiother. Oncol. J. Eur. Soc. Ther. Radiol. Oncol. 108 (2013) 136-142. doi:10.1016/j.radonc.2013.04.004.

[113] T. Guo, X-Ray Nanochemistry: Background and Introduction, in: T. Guo (Ed.), X-Ray Nanochemistry Concepts Dev., Springer International Publishing, Cham, 2018: pp. 3-20. doi:10.1007/978-3-319-78004-7_1.

[114] T. Guo, Physical Enhancement of the Effectiveness of X-Ray Irradiation, in: T. Guo (Ed.), X-Ray Nanochemistry Concepts Dev., Springer International Publishing, Cham, 2018: pp. 23-116. doi:10.1007/978-3-319-78004-7_2. 
[115] J.H. Hubell, Photon Mass Attenuation and Mass Energy-Absorption Coefficients for H, C, N, O, Ar, and Seven Mixtures from $0.1 \mathrm{keV}$ to $20 \mathrm{MeV}$, Radiat. Res. 70 (1977) 58-81. doi: $10.2307 / 3574732$.

[116] T. Guo, Chemical Enhancement, in: T. Guo (Ed.), X-Ray Nanochemistry Concepts Dev., Springer International Publishing, Cham, 2018: pp. 117-157. doi:10.1007/978-3-319-780047_3.

[117] N.N. Cheng, Z. Starkewolf, R.A. Davidson, A. Sharmah, C. Lee, J. Lien, T. Guo, Chemical Enhancement by Nanomaterials under X-ray Irradiation, J. Am. Chem. Soc. 134 (2012) 1950-1953. doi:10.1021/ja210239k.

[118] C. Sicard-Roselli, E. Brun, M. Gilles, G. Baldacchino, C. Kelsey, H. McQuaid, C. Polin, N. Wardlow, F. Currell, A New Mechanism for Hydroxyl Radical Production in Irradiated Nanoparticle Solutions, Small. 10 (2014) 3338-3346. doi:10.1002/smll.201400110.

[119] H.D. Jang, S.-K. Kim, S.-J. Kim, Effect of Particle Size and Phase Composition of Titanium Dioxide Nanoparticles on the Photocatalytic Properties, J. Nanoparticle Res. 3 (2001) 141-147. doi:10.1023/A:1017948330363.

[120] Z. Niu, Y. Li, Removal and Utilization of Capping Agents in Nanocatalysis, Chem. Mater. 26 (2014) 72-83. doi:10.1021/cm4022479.

[121] T. Guo, Biological Enhancement of X-Ray Effects, in: T. Guo (Ed.), X-Ray Nanochemistry Concepts Dev., Springer International Publishing, Cham, 2018: pp. 159-176. doi:10.1007/978-3-319-78004-7_4.

[122] A. Corma, H. Garcia, Supported gold nanoparticles as catalysts for organic reactions, Chem. Soc. Rev. 37 (2008) 2096. doi:10.1039/b707314n. 
[123] B. Gupta, A.A. Melvin, T. Matthews, S. Dash, A.K. Tyagi, TiO2 modification by gold (Au) for photocatalytic hydrogen (H2) production, Renew. Sustain. Energy Rev. 58 (2016) 1366-1375. doi:10.1016/j.rser.2015.12.236.

[124] H. Xu, B.W. Zeiger, K.S. Suslick, Sonochemical synthesis of nanomaterials, Chem Soc Rev. 42 (2013) 2555-2567. doi:10.1039/C2CS35282F.

[125] Y. Mizukoshi, Y. Makise, T. Shuto, J. Hu, A. Tominaga, S. Shironita, S. Tanabe, Immobilization of noble metal nanoparticles on the surface of $\mathrm{TiO} 2$ by the sonochemical method: Photocatalytic production of hydrogen from an aqueous solution of ethanol, Ultrason. Sonochem. 14 (2007) 387-392. doi:10.1016/j.ultsonch.2006.08.001.

[126] J.H. Bang, K.S. Suslick, Applications of Ultrasound to the Synthesis of Nanostructured Materials, Adv. Mater. 22 (2010) 1039-1059. doi:10.1002/adma.200904093.

[127] J. Cihlar, E. Bartonickova, J. Cihlar, Low-temperature sol-gel synthesis of anatase nanoparticles modified by $\mathrm{Au}, \mathrm{Pd}$ and $\mathrm{Pt}$ and activity of TiO2/Au, Pd, Pt photocatalysts in water splitting, J. Sol-Gel Sci. Technol. 65 (2013) 430-442. doi:10.1007/s10971-012-29558.

[128] D. Ficai, A.M. Grumezescu, Synthetic Methods for Preparation of Metal Nanoparticles, in: $\begin{array}{llll}\text { Nanostructures } & \text { Nov. } & \text { Ther., } & \text { Elsevier, }\end{array}$ https://proquest.safaribooksonline.com/book/nanotechnology/9780323461481/chapter-1novel-approaches-for-preparation-of-nanoparticles/st0035_html (accessed February 28, 2019).

[129] J. Fang, S.-W. Cao, Z. Wang, M.M. Shahjamali, S.C.J. Loo, J. Barber, C. Xue, Mesoporous plasmonic $\mathrm{Au}-\mathrm{TiO} 2$ nanocomposites for efficient visible-light-driven photocatalytic water 
reduction, Int. J. Hydrog. Energy. $37 \quad$ (2012) 17853-17861. doi:10.1016/j.ijhydene.2012.09.023.

[130] Sia Zhang, J. Liu, G.Q. (Max) Lu, Synthetic chemistry of nanomaterials, in: Mod. Inorg. Synth. Chem., Elservier, Oxford, UK, n.d.: pp. 479-590.

[131] M.L. Marin, K.L. McGilvray, J.C. Scaiano, Photochemical Strategies for the Synthesis of Gold Nanoparticles from $\mathrm{Au}(\mathrm{III})$ and $\mathrm{Au}(\mathrm{I})$ Using Photoinduced Free Radical Generation, J. Am. Chem. Soc. 130 (2008) 16572-16584. doi:10.1021/ja803490n.

[132] A.A. Zezin, V.I. Feldman, A.V. Dudnikov, S.B. Zezin, S.S. Abramchuk, S.I. Belopushkin, Reduction of copper(II) ions in polyacrylic acid-polyethyleneimine complexes using X-ray radiation, High Energy Chem. 43 (2009) 100-104. doi:10.1134/S0018143909020064.

[133] F. Muller, P. Fontaine, S. Remita, M.-C. Fauré, E. Lacaze, M. Goldmann, Synthesis of Nanostructured Metal-Organic Films: Surface X-ray Radiolysis of Silver Ions Using a Langmuir Monolayer as a Template, Langmuir. $20 \quad$ (2004) 4791-4794. doi:10.1021/la049534u.

[134] Y. Ohkubo, T. Nakagawa, S. Seino, J. Kugai, T.A. Yamamoto, H. Nitani, Y. Niwa, X-rayinduced reduction of Au ions in an aqueous solution in the presence of support materials and in situ time-resolved XANES measurements, J. Synchrotron Radiat. 21 (2014) 1148-1152. doi:10.1107/S1600577514012703.

[135] I.V. Gala, Nuevos tratamientos de aguas mediante tecnologías integradas basadas en el uso de procesos avanzados de oxidación/reducción y carbón activado, http://purl.org/dc/dcmitype/Text, Universidad de $\quad 2013$. https://dialnet.unirioja.es/servlet/tesis?codigo=59225 (accessed August 31, 2017). 
[136] W.M. Haynes, CRC Handbook of Chemistry and Physics, 91st Edition, Taylor \& Francis Group, 2010.

[137] A. Abedini, F. Larki, E. Saion, A. Zakaria, M. Zobir Hussein, Influence of dose and ion concentration on formation of binary Al-Ni alloy nanoclusters, Radiat. Phys. Chem. 81 (2012) 1653-1658. doi:10.1016/j.radphyschem.2012.05.015.

[138] J. Grand, S.R. Ferreira, V. de Waele, S. Mintova, T.M. Nenoff, Nanoparticle Alloy Formation by Radiolysis, J. Phys. Chem. C. 122 (2018) 12573-12588. doi:10.1021/acs.jpcc.8b01878.

[139] H.S. Dehsari, A.H. Ribeiro, B. Ersöz, W. Tremel, G. Jakob, K. Asadi, Effect of precursor concentration on size evolution of iron oxide nanoparticles, CrystEngComm. 19 (2017) 6694-6702. doi:10.1039/C7CE01406F.

[140] A.Y. Kuznetsov, R. Machado, L.S. Gomes, C.A. Achete, V. Swamy, B.C. Muddle, V. Prakapenka, Size dependence of rutile $\mathrm{TiO} 2$ lattice parameters determined via simultaneous size, strain, and shape modeling, Appl. Phys. Lett. 94 (2009) 193117. doi:10.1063/1.3139078.

[141] J. Khatouri, M. Mostafavi, J. Belloni, Kinetics of Electron Transfer in Solution Catalyzed by Metal Clusters, in: Photochem. Radiat. Chem., American Chemical Society, 1998: pp. 293-314. doi:10.1021/ba-1998-0254.ch018.

[142] R. Zanella, S. Giorgio, C.-H. Shin, C.R. Henry, C. Louis, Characterization and reactivity in $\mathrm{CO}$ oxidation of gold nanoparticles supported on $\mathrm{TiO} 2$ prepared by depositionprecipitation with $\mathrm{NaOH}$ and urea, J. Catal. 222 (2004) 357-367. doi:10.1016/j.jcat.2003.11.005.

[143] W. Brack, B. Heine, F. Birkhold, M. Kruse, G. Schoch, S. Tischer, O. Deutschmann, Kinetic modeling of urea decomposition based on systematic thermogravimetric analyses of 
urea and its most important by-products, Chem. Eng. Sci. 106 (2014) 1-8. doi:10.1016/j.ces.2013.11.013.

[144] M.P Casaletto, A. Longo, A. Martorana, A. Prestianni, A.M. Venezia, XPS study of supported gold catalysts: the role of $\mathrm{Au} 0$ and $\mathrm{Au}+\delta$ species as active sites, Surf. Interface Anal. 38 (2006) 215-218. doi:10.1002/sia.2180.

[145] M. Haruta, When Gold Is Not Noble: Catalysis by Nanoparticles, Chem. Rec. 3 (2003) 7587. doi:10.1002/tcr.10053.

[146] M.C. Molina Higgins, J.V. Rojas, X-ray radiation enhancement of gold- TiO2 nanocomposites, Appl. Surf. Sci. 480 (2019) 1147-1155. doi:10.1016/j.apsusc.2019.02.234.

[147] L. Samet, K. March, O. Stephan, N. Brun, F. Hosni, F. Bessousa, J. Benasseur, R. Chtourou, Radiocatalytic $\mathrm{Cu}$-incorporated $\mathrm{TiO} 2$ nano-particles for the degradation of organic species under gamma irradiation, J. Alloys Compd. 743 (2018) 175-186. doi:10.1016/j.jallcom.2018.02.001.

[148] C. Yogi, K. Kojima, T. Takai, N. Wada, Photocatalytic degradation of methylene blue by Au-deposited TiO2 film under UV irradiation, J. Mater. Sci. 44 (2009) 821. doi:10.1007/s10853-008-3151-7.

[149] M. Gilles, E. Brun, C. Sicard-Roselli, Quantification of hydroxyl radicals and solvated electrons produced by irradiated gold nanoparticles suggests a crucial role of interfacial water, J. Colloid Interface Sci. 525 (2018) 31-38. doi:10.1016/j.jcis.2018.04.017.

[150] J.A. LaVerne, L. Tandon, B.C. Knippel, V.M. Montoya, Heavy ion radiolysis of methylene blue, Radiat. Phys. Chem. 72 (2005) 143-147. doi:10.1016/j.radphyschem.2004.09.010.

[151] A. Primo, A. Corma, H. García, Titania supported gold nanoparticles as photocatalyst, Phys. Chem. Chem. Phys. 13 (2010) 886-910. doi:10.1039/C0CP00917B. 
[152] Y. Yin, Y. Zhang, M. Wang, K. Shi, Effects of temperature on the optical properties of Microcystis aeruginosa and Scenedesmus obliquus, J. Freshw. Ecol. 31 (2016) 361-375. doi:10.1080/02705060.2016.1151467.

[153] Y. Wan, X. Zhou, Formation mechanism of hafnium oxide nanoparticles by a hydrothermal route, RSC Adv. 7 (2017) 7763-7773. doi:10.1039/C6RA26663K.

[154] S.G. Kumar, K.S.R.K. Rao, Comparison of modification strategies towards enhanced charge carrier separation and photocatalytic degradation activity of metal oxide semiconductors (TiO 2, WO 3 and ZnO), Appl. Surf. Sci. 391 (2017) 124-148. doi:10.1016/j.apsusc.2016.07.081.

[155] B. Banerjee, Recent developments on nano-ZnO catalyzed synthesis of bioactive heterocycles, J. Nanostructure Chem. 7 (2017) 389-413. doi:10.1007/s40097-017-0247-0.

[156] C.L. Tourneau, V. Calugaru, J.O. Thariat, C. Florescu, X. Mirabel, F. Jegoux, T. Jouffroy, J. Rodriguez, C. Hoffmann, B. Dodger, V.M. Garcia, M. Dimitriu, L. Levy, E. Calvo, Hafnium Oxide Nanoparticles as a Promising Emergent Treatment for Head And Neck Cancer, Int. J. Radiat. Oncol. • Biol. • Phys. $100 \quad$ (2018) 1377. doi:10.1016/j.jjrobp.2017.12.180.

[157] V. Jayaraman, G. Bhavesh, S. Chinnathambi, S. Ganesan, P. Aruna, Synthesis and characterization of hafnium oxide nanoparticles for bio-safety, (2014). doi:info:doi/10.1166/mex.2014.1190.

[158] H. Hinterwirth, S. Kappel, T. Waitz, T. Prohaska, W. Lindner, M. Lämmerhofer, Quantifying Thiol Ligand Density of Self-Assembled Monolayers on Gold Nanoparticles by Inductively Coupled Plasma-Mass Spectrometry, ACS Nano. 7 (2013) 1129-1136. doi:10.1021/nn306024a. 
[159] A. Franco, A.M. Balu, A.A. Romero, R. Luque, Nanostructured Porous Materials: Synthesis and Catalytic Applications, Nanotechnol. Catal. (2017). doi:10.1002/9783527699827.ch6.

[160] M. Anjum, R. Miandad, M. Waqas, F. Gehany, M.A. Barakat, Remediation of wastewater using various nano-materials, Arab. J. Chem. (2016). doi:10.1016/j.arabjc.2016.10.004.

[161] R.N. Bharagava, Recent Advances in Environmental Management, CRC Press, 2018.

[162] S.P. Sahu, E.L. Cates, X-ray Radiocatalytic Activity and Mechanisms of Bismuth Complex Oxides, (2017). doi:10.1021/acs.jpcc.7b00776.

[163] P. Sangpour, F. Hashemi, A.Z. Moshfegh, Photoenhanced Degradation of Methylene Blue on Cosputtered M:TiO2 ( $\mathrm{M}=\mathrm{Au}, \mathrm{Ag}, \mathrm{Cu}$ ) Nanocomposite Systems: A Comparative Study, J. Phys. Chem. C. 114 (2010) 13955-13961. doi:10.1021/jp910454r.

[164] I. Tanabe, T. Ryoki, Y. Ozaki, The effects of Au nanoparticle size (5-60 nm) and shape (sphere, rod, cube) over electronic states and photocatalytic activities of $\mathrm{TiO} 2$ studied by farand deep-ultraviolet spectroscopy, RSC Adv. 5 (2015) 13648-13652. doi:10.1039/C4RA12503G. 
VITA

MARIA MOLINA HIGGINS

\section{EDUCATION}

- Virginia Commonwealth University (MAy 2019)

$\mathrm{PhD}$ Candidate in Mechanical \& Nuclear Engineering Department

- Universidad NACIONAL de COlOMbia (APRIL 2014)

Bachelors of Science in Mechanical Engineering

\section{PUBLICATIONS}

- M.C Molina Higgins, J.V Rojas. X-ray Radiation Enhancement of Gold-TiO 2 Nanocomposites. Applied Surface Science. pp 1147-1155. February 2019.

- M.C Molina Higgins, M. Toro Gonzalez, J.V Rojas, Enhanced X-rays degradation of methylene blue in the presence of gold microspheres. Radiation Physics and Chemistry. pp.73-80. October 2018.

- M.C Molina Higgins, D.C Clifford, J.V. Rojas, Au@TiO2 Nanocomposites Synthesized by X-ray Radiolysis as Potential Radiosensitizers. Applied Surface Science. pp. 702-710, 2017

- J.V Rojas, M.C Molina-Higgins, M. Toro-Gonzalez and C. Castano, "Single step radiolytic synthesis of iridium nanoparticles onto graphene oxide," Applied Surface Science, pp. 20872093, 2015. 
- J.V Rojas, M. Toro-Gonzalez, M.C Molina-Higgins and C. Castano, "Facile radiolytic synthesis of ruthenium nanoparticles on graphene oxide and carbon nanotubes," Materials Science \& Engineering B, pp. pp.28-35, 2016.

\section{CONFERENCE PROCEEDINGS}

- M.C Molina Higgins and J. Rojas, Synthesis of $\mathrm{Au} @ \mathrm{TiO}_{2} \mathrm{NCs}$ by X-ray radiolytic and Deposition-Precipitation methods, in ANS, 2017.

- M. Toro-González, M.C Molina Higgins and J.V Rojas. Influence of Gold Particle Concentration and X-ray Energy in Radiosensitization, in ANS, 2017

- M.C Molina Higgins and J.V Rojas, Evaluation of $\mathrm{TiO}_{2}$ and $\mathrm{Au} @ \mathrm{TiO}_{2}$ nanoparticles as a Potential Radiosensitizers by Decomposition of Methylene Blue, Poster Session ANS Winter, 2016.

- M.C Molina-Higgins and J.V Rojas, "Growth Kinetics of Lanthanum Phosphate Core/Shell Nanoparticles Doped with $\mathrm{Ce}-\mathrm{Tb}$ and Eu," in Processing, Properties, and Design of Advanced Ceramics and Composites: Ceramic Transactions, 2016 